\title{
A novel approach to study atmospheric neutrino oscillation
}

\author{
Shao-Feng Ge, ${ }^{a}$ Kaoru Hagiwara ${ }^{b}$ and Carsten $\operatorname{Rott}^{c}$ \\ ${ }^{a}$ KEK Theory Center, \\ Tsukuba, 305-0801, Japan \\ ${ }^{b}$ KEK Theory Center and Sokendai, \\ Tsukuba, 305-0801, Japan \\ ${ }^{c}$ Department of Physics, Sungkyunkwan University, \\ Suwon 440-746, Korea \\ E-mail: gesf02@gmail.com, kaoru.hagiwara@kek.jp, \\ carsten.rott@gmail.com
}

ABSTRACT: We develop a general theoretical framework to analytically disentangle the contributions of the neutrino mass hierarchy, the atmospheric mixing angle, and the CP phase, in neutrino oscillations. To illustrate the usefulness of this framework, especially that it can serve as a complementary tool to neutrino oscillogram in the study of atmospheric neutrino oscillations, we take PINGU as an example and compute muon- and electron-like event rates with event cuts on neutrino energy and zenith angle. Under the assumption of exact measurement of neutrino momentum with a perfect e- $\mu$ identification and no background, we find that the PINGU experiment has the potential of resolving the neutrino mass hierarchy and the octant degeneracies within 1-year run, while the measurement of the CP phase is significantly more challenging. Our observation merits a serious study of the detector capability of estimating the neutrino momentum for both muon- and electron-like events.

Keywords: Neutrino Physics, Solar and Atmospheric Neutrinos

ARXIV EPRINT: 1309.3176 


\section{Contents}

1 Introduction 1

2 Disentangling parameters in the propagation basis 3

2.1 Propagation basis 3

2.2 Oscillation probabilities 5

2.3 Simplifications with symmetric matter profile 6

2.4 Expansion of oscillation probabilities with respect to $x_{\mathrm{a}}=\cos 2 \theta_{\mathrm{a}}$ and $\delta m_{\mathrm{s}}^{2} \quad 7$

3 Event rates at a charge-blind detector 12

$\begin{array}{lll}4 & \text { A simple } \chi^{2} \text { analysis } & 17\end{array}$

$4.1 \chi^{2}$ function 18

$\begin{array}{ll}4.2 \text { The mass hierarchy } & 19\end{array}$

4.3 The atmospheric angle and its octant 21

$\begin{array}{lll}\text { 4.3.1 The atmospheric angle } & 22\end{array}$

$\begin{array}{lll}4.3 .2 & \text { Octant sensitivity } & 23\end{array}$

4.4 Uncertainty of the CP phase 25

5 Conclusions and outlook $\quad 27$

A Atmospheric neutrino oscillation $\quad \mathbf{2 8}$

A.1 Atmospheric neutrino flux, cross sections and effective volume 28

A.2 Earth matter profile 30

A.3 Numerical method for oscillation amplitude matrix $S^{\prime}$ in the propagation basis 32

A.4 Normal hierarchy vs. inverted hierarchy 33

\section{Introduction}

In the last two years, the field of neutrino physics has significantly advanced by constraining the reactor angle $\theta_{13}$. The T2K experiment [1] was the first to report a hint of nonzero reactor angle, followed by MINOS [2] and Double CHOOZ [3] which added up to a confidence level above 3 sigma. It was measured accurately by Daya Bay [4] and RENO [5] in March and April 2012, respectively, reaching 7.7 sigma [6] by the October of the same year.

The relatively large reactor angle opens up opportunities [7] for determining the mass hierarchy, the octant of the atmospheric mixing angle, and the CP phase. The first could be achieved with a medium baseline reactor experiment [8-21] and long baseline accelerator experiments [22-37, 44] could measure all three of them. Atmospheric neutrino experiments [38-71] could offer alternative ways to accomplish the same. 
Recent studies have focused on magnetized detectors, which can distinguish neutrinos from antineutrinos [44, 48, 49, 51]. Equipped with this capability, a detector of 50100 Kton $\left(\sim 10^{3}\right.$ tons $)$ scale is enough to distinguish the mass hierarchy. Large volume water-Cherenkov or ice-Cherenkov detectors of tens of Mton ( $\sim 10^{6}$ tons) scale could offer an alternative. DeepCore [52], the existing in-fill to IceCube can reach down to energies of $\mathcal{O}(10) \mathrm{GeV}$ and has recently reported the observation of muon neutrino disappearance oscillations [53] and an electron neutrino flux consistent with expectations [54, 55], demonstrating the capabilities of an low-energy extension. DeepCore has also some sensitivity to neutrinos from the MSW resonance region [73-76] around $E_{\nu} \approx 5 \sim 10 \mathrm{GeV}$. It can however only partially cover it $[47,50]$ and to really exploit it a lower threshold detector would be needed.

There has been extensive interest [66-70, 77-81] recently by the IceCube Collaboration and theoretical community to extend the existing IceCube neutrino telescope [56] with an in-fill array called PINGU (Precision Icecube Next Generation Upgrade) [57] that could detect neutrino events of $\mathcal{O}(1) \mathrm{GeV}$. Such a detector opens up the opportunity of detecting more patterns of the atmospheric neutrino oscillation behavior, which is diluted at higher energy scale, especially due to matter effects [59-65]. A large benefit is the expected high event statistics at low energies. Event rates of $\mathcal{O}(100,000)$ per year from atmospheric neutrinos allow for measurements with small statistical uncertainty. During the preparation of this draft, a preliminary experimental study [84] appeared. In Europe a similar detector to PINGU is being considered as part of the Km3NeT project. Our studies can be transferred to this ORCA (Oscillation Research with Cosmics in the Abyss) [85].

The expectation of a high statistics sample down to $1 \mathrm{GeV}$ scale makes determining the mixing parameters with atmospheric neutrinos very promising $[47,50,66,68]$. The paper [66, 68, 69] adopts oscillograms [59-65] to depict the structure of oscillation resonances [61-63, 73-76, 86-95] when atmospheric neutrinos travel through the Earth. The ability to determine the mass hierarchy, the octant of the atmospheric mixing angle, and the CP phase is studied. The event numbers and difference between normal hierarchy (NH) and inverted hierarchy ( $\mathrm{IH})$ are shown in oscillograms. In [71], the Bayesian approach is explored in a generic way while the Toy Monte Carlo based on an extended unbinned likelihood ratio test statistic is implemented in [72]. When combined with accelerator experiments, the sensitivities on the octant of the atmospheric mixing angle $[38,39,51,66,96]$ and the CP phase [97] can be enhanced.

In section 2, we first develop a general framework of decomposing the neutrino oscillation probabilities and the event rates in the propagation basis, and apply it to the symmetric Earth matter profile in order to analytically disentangle the effects of the neutrino mass hierarchy, the atmospheric angle, and the $\mathrm{CP}$ phase. In section 3 , we calculate and display the event rates that can be observed at PINGU. Based on these results, we try to establish the potential of atmospheric neutrino measurement at PINGU in section 4, while its dependence on the input values of the neutrino mass hierarchy, the atmospheric mixing angle and the CP phase can be fully understood in our decomposition formalism. Finally, we summarize our conclusions in section 5. For more details about the basic inputs, including the atmospheric neutrino fluxes, cross sections, effective fiducial volume of 
PINGU, and the Earth matter profile, as well as the numerical methods of evaluating the neutrino oscillation probabilities through Earth, please refer to section A.

\section{Disentangling parameters in the propagation basis}

We first develop a general framework in the propagation basis $[106,107]$ for phenomenological study of neutrino oscillation. It can analytically decompose the contributions of the neutrino mass hierarchy, the atmospheric mixing angle, and the CP phase. This decomposition method can serve as a complementary tool to the neutrino oscillogram [59-65] for the analysis of atmospheric neutrino oscillations, and can apply generally to other types of neutrino oscillation experiments.

\subsection{Propagation basis}

In the propagation basis, the atmospheric mixing angle $\theta_{23}$ [106] and the CP phase $\delta$ [107] can be disentangled from the other mixing parameters as well as the Earth matter potential [108]. This can be seen from the effective Hamiltonian,

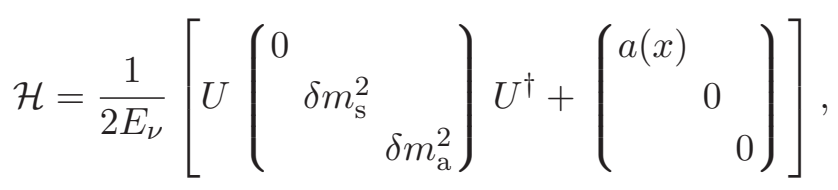

where

$$
a(x) \equiv 2 E_{\nu} V(x)=2 \sqrt{2} E_{\nu} G_{F} N_{\mathrm{e}}(x),
$$

represents the matter effect which is proportional to neutrino energy $E_{\nu}$ and the matter potential $V(x)$. The mass differences are denoted as,

$$
\delta m_{\mathrm{s}}^{2} \equiv m_{2}^{2}-m_{1}^{2}, \quad \delta m_{\mathrm{a}}^{2} \equiv m_{3}^{2}-m_{1}^{2} .
$$

The lepton-flavor mixing matrix $U$ relates the flavor basis $\left(\nu_{\alpha}=\nu_{\mathrm{e}}, \nu_{\mu}, \nu_{\tau}\right)$ and the mass eigenstates $\left(m_{\nu_{i}}=m_{i}, i=1,2,3\right)$,

$$
\nu_{\alpha}=U_{\alpha i} \nu_{i}
$$

and can be parametrized as $U \equiv O_{23}\left(\theta_{\mathrm{a}}\right) P_{\delta} O_{13}\left(\theta_{\mathrm{r}}\right) P_{\delta}^{\dagger} O_{12}\left(\theta_{\mathrm{s}}\right)$,

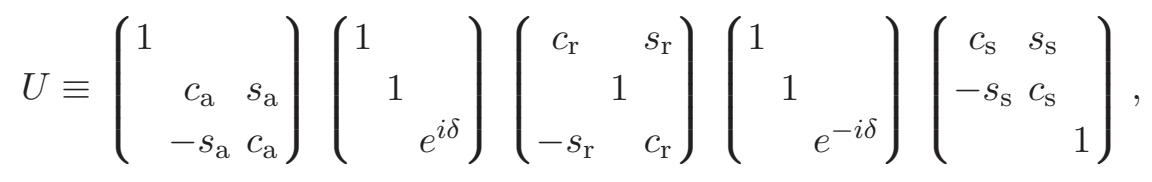

where $c_{\alpha} \equiv \cos \theta_{\alpha}$ and $s_{\alpha} \equiv \sin \theta_{\alpha}$. The solar, the atmospheric, and the reactor mixing angles are labelled as,

$$
(s, a, r) \equiv(12,23,13),
$$

according to how they were measured. For convenience, we denote the three rotation matrices in (2.5) from the left to the right as $O_{23}, O_{13}$, and $O_{12}$ respectively. 
The 2-3 mixing matrix $O_{23}$ and the rephasing matrix $P_{\delta}$ can be extracted out as overall matrices [107, 108],

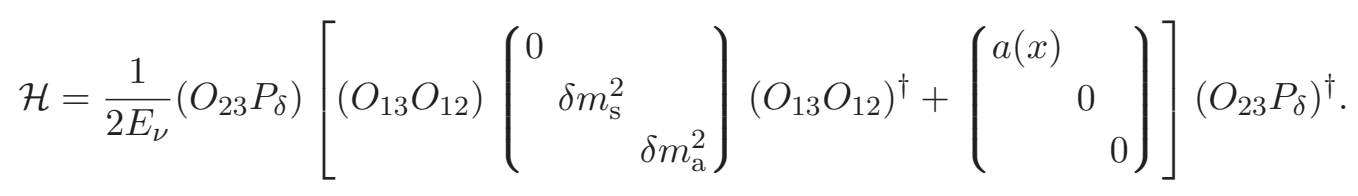

In this way, $O_{23}$ and $P_{\delta}$ are separated from the neutrino mass hierarchy, which is encoded in the first term inside the square bracket, as well as the matter effect, represented by the second term. In other words, the atmospheric mixing angle $\theta_{\mathrm{a}}$ and the $\mathrm{CP}$ phase $\delta$ are disentangled from the remaining mixing parameters, analytically. This is a significant simplification in the analysis of neutrino oscillation, especially the atmospheric neutrino oscillation that suffers from complicated matter profile, as described in section A.2.

To make it explicit, the original Hamiltonian $\mathcal{H}$ can be rotated to the equivalent $\mathcal{H}^{\prime}$ in the propagation basis through a similar transformation,

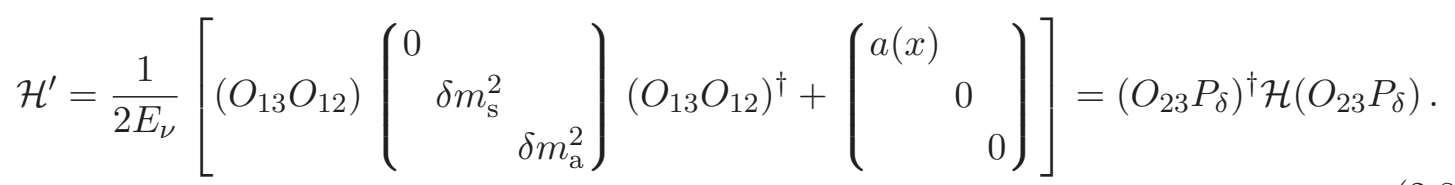

There are only four mixing parameters involved in $\mathcal{H}^{\prime}$, the two mass squared differences $\delta m_{\mathrm{s}}^{2}$ and $\delta m_{\mathrm{a}}^{2}$, the solar mixing angle $\theta_{\mathrm{s}}$, and the reactor mixing angle $\theta_{\mathrm{r}}$. Correspondingly, we can define a propagation basis $\left(\nu_{i}^{\prime}\right)[106,107]$ that is related to the flavor basis $\left(\nu_{\alpha}\right)$ and the mass eigenstates $\left(\nu_{i}\right)$ as follows:

$$
\nu_{\alpha}=\left[O_{23}\left(\theta_{\mathrm{a}}\right) P_{\delta}\right]_{\alpha i} \nu_{i}^{\prime} .
$$

The transformed Hamiltonian $\mathcal{H}^{\prime}$ is the effective Hamiltonian defined in the propagation basis. Once the neutrino oscillation amplitudes,

$$
S_{\mathrm{ij}}^{\prime} \equiv\left\langle\nu_{j}^{\prime}\left|S^{\prime}\right| \nu_{i}^{\prime}\right\rangle
$$

are calculated with the Hamiltonian $\mathcal{H}^{\prime}$ in the propagation basis, the oscillation amplitudes in the flavor basis,

$$
S_{\alpha \beta} \equiv\left\langle\nu_{\beta}|S| \nu_{\alpha}\right\rangle
$$

are simply obtained by the unitarity transformation,

$$
S=\left(O_{23} P_{\delta}\right) S^{\prime}\left(O_{23} P_{\delta}\right)^{\dagger} \equiv\left(O_{23} P_{\delta}\right)\left(\begin{array}{lll}
S_{11}^{\prime} & S_{12}^{\prime} & S_{13}^{\prime} \\
S_{21}^{\prime} & S_{22}^{\prime} & S_{23}^{\prime} \\
S_{31}^{\prime} & S_{32}^{\prime} & S_{33}^{\prime}
\end{array}\right)\left(O_{23} P_{\delta}\right)^{\dagger}
$$

This makes the formalism much simpler.

We find the propagation basis very useful in the phenomenological study of neutrino oscillation. It allows us to analytically factor out $\theta_{\mathrm{a}}$ [106] and $\delta$ [107] from the numerical evaluation of the oscillation amplitudes that involves many factors and can be very complicated [108]. The contributions of the still unknown neutrino mass hierarchy, the octant of 
the atmospheric mixing angle $\theta_{\mathrm{a}}$, and the $\mathrm{CP}$ phase $\delta$ are now disentangled from each other. A general formalism based on this feature can help to reveal the pictures behind neutrino oscillation phenomena. This is especially important when the three unknown parameters are under close investigations at current and future neutrino experiments.

\subsection{Oscillation probabilities}

The oscillation probabilities are measured in the flavor basis. It is necessary to explicitly express the flavor basis amplitude matrix $S$ in terms of its counterpart $S^{\prime}$ in the propagation basis by the unitary transformation with $O_{23} P_{\delta}$, namely to expand (2.12). According to the definition of the mixing matrix in (2.5), $O_{23} P_{\delta}$ can be explicitly written as,

$$
O_{23} P_{\delta}=\left(\begin{array}{ccc}
1 & & \\
& c_{\mathrm{a}} & s_{\mathrm{a}} e^{i \delta} \\
& -s_{\mathrm{a}} & c_{\mathrm{a}} e^{i \delta}
\end{array}\right), \quad\left(O_{23} P_{\delta}\right)^{\dagger}=\left(\begin{array}{ccc}
1 & & \\
& c_{\mathrm{a}} & -s_{\mathrm{a}} \\
& s_{\mathrm{a}} e^{-i \delta} & c_{\mathrm{a}} e^{-i \delta}
\end{array}\right) .
$$

The mixing from the propagation to the flavor basis occurs between the second and the third indices. We can expect the first element of $S^{\prime}$ to be unaffected when (2.13) is combined with (2.12) [107],

$$
\begin{aligned}
S_{\mathrm{ee}} & =S_{11}^{\prime}, \\
S_{\mathrm{e} \mu} & =c_{\mathrm{a}} S_{12}^{\prime}+s_{\mathrm{a}} e^{-i \delta} S_{13}^{\prime}, \\
S_{\mu \mathrm{e}} & =c_{\mathrm{a}} S_{21}^{\prime}+s_{\mathrm{a}} e^{+i \delta} S_{31}^{\prime}, \\
S_{\mu \mu} & =c_{\mathrm{a}}^{2} S_{22}^{\prime}+c_{\mathrm{a}} s_{\mathrm{a}}\left(e^{-i \delta} S_{23}^{\prime}+e^{+i \delta} S_{32}^{\prime}\right)+s_{\mathrm{a}}^{2} S_{33}^{\prime} .
\end{aligned}
$$

Note that only the elements among $e$ and $\mu$ flavors are shown since they are sufficient to derive all the flavor basis oscillation probabilities,

$$
P_{\alpha \beta} \equiv P\left(\nu_{\alpha} \rightarrow \nu_{\beta}\right)=\left|\left\langle\nu_{\beta}|S| \nu_{\alpha}\right\rangle\right|^{2}=\left|S_{\beta \alpha}\right|^{2},
$$

from $\nu_{\mathrm{e}}$ and $\nu_{\mu}$ (as well as from $\bar{\nu}_{\mathrm{e}}$ and $\bar{\nu}_{\mu}$, as shown below). Explicitly we find,

$$
\begin{aligned}
P_{\mathrm{ee}} \equiv\left|S_{\mathrm{ee}}\right|^{2}= & \left|S_{11}^{\prime}\right|^{2}, \\
P_{\mathrm{e} \mu} \equiv\left|S_{\mu \mathrm{e}}\right|^{2}= & c_{\mathrm{a}}^{2}\left|S_{12}^{\prime}\right|^{2}+s_{\mathrm{a}}^{2}\left|S_{13}^{\prime}\right|^{2}+2 c_{\mathrm{a}} s_{\mathrm{a}}(\cos \delta \mathbb{R}+\sin \delta \mathbb{I})\left(S_{12}^{\prime} S_{13}^{\prime *}\right), \\
P_{\mu \mathrm{e}} \equiv\left|S_{\mathrm{e} \mu}\right|^{2}= & c_{\mathrm{a}}^{2}\left|S_{21}^{\prime}\right|^{2}+s_{\mathrm{a}}^{2}\left|S_{31}^{\prime}\right|^{2}+2 c_{\mathrm{a}} s_{\mathrm{a}}(\cos \delta \mathbb{R}-\sin \delta \mathbb{I})\left(S_{21}^{\prime} S_{31}^{\prime *}\right), \\
P_{\mu \mu} \equiv\left|S_{\mu \mu}\right|^{2}= & c_{\mathrm{a}}^{4}\left|S_{22}^{\prime}\right|^{2}+s_{\mathrm{a}}^{4}\left|S_{33}^{\prime}\right|^{2}+2 c_{\mathrm{a}}^{2} s_{\mathrm{a}}^{2} \mathbb{R}\left(S_{22}^{\prime} S_{33}^{\prime *}\right) \\
& +c_{\mathrm{a}}^{2} s_{\mathrm{a}}^{2}\left[\left|S_{23}^{\prime}\right|^{2}+2(\cos 2 \delta \mathbb{R}+\sin 2 \delta \mathbb{I})\left(S_{23}^{\prime} S_{32}^{\prime *}\right)+\left|S_{32}^{\prime}\right|^{2}\right] \\
& +2 c_{\mathrm{a}} s_{\mathrm{a}} \cos \delta \mathbb{R}\left[\left(c_{\mathrm{a}}^{2} S_{22}^{\prime}+s_{\mathrm{a}}^{2} S_{33}^{\prime}\right)\left(S_{23}^{\prime}+S_{32}^{\prime}\right)^{*}\right] \\
& +2 c_{\mathrm{a}} s_{\mathrm{a}} \sin \delta \mathbb{I}\left[\left(c_{\mathrm{a}}^{2} S_{22}^{\prime}+s_{\mathrm{a}}^{2} S_{33}^{\prime}\right)\left(S_{32}^{\prime}-S_{23}^{\prime}\right)^{*}\right],
\end{aligned}
$$

where $\mathbb{R}$ and $\mathbb{I}$ gives the real and imaginary parts, respectively. The dependence on the atmospheric mixing angle $\theta_{\mathrm{a}}$ and the CP phase $\delta$ can be clearly seen in the above expressions. The transition probability into $\nu_{\tau}$ are then obtained by unitarity conditions,

$$
\begin{gathered}
P_{\mathrm{e} \tau}=1-P_{\mathrm{ee}}-P_{\mathrm{e} \mu}, \\
P_{\mu \tau}=1-P_{\mu \mathrm{e}}-P_{\mu \mu},
\end{gathered}
$$


while we neglect contributions from tiny components of $\nu_{\tau}$ and $\bar{\nu}_{\tau}$ flux in the atmospheric neutrinos [102].

The oscillation probabilities for antineutrinos are then obtained simply as,

$$
\bar{P}_{\alpha \beta} \equiv P\left(\bar{\nu}_{\alpha} \rightarrow \bar{\nu}_{\beta}\right)=P_{\alpha \beta}(a(x) \rightarrow-a(x), \delta \rightarrow-\delta),
$$

by reversing the sign of the matter potential in the Hamiltonian (2.1) and the CP phase $\delta$ in the neutrino mixing matrix (2.5), which is identical to the parametrization adopted in Review of Particle Physics [109].

\subsection{Simplifications with symmetric matter profile}

The expressions in (2.16) can be significantly simplified in the approximation of the symmetric or reversible matter profile along the baseline, such as those of atmospheric neutrinos in the earth whose matter profile is approximately spherically symmetric as in PREM [98] adopted in our study. It has been known that [110] the oscillation amplitude matrix after experiencing a reversible matter profile is symmetric in the absence of $\mathrm{CP}$ violation. This is indeed the case for the oscillation amplitudes through the Earth in the propagation basis, giving,

$$
S_{\mathrm{ij}}^{\prime}=S_{\mathrm{ji}}^{\prime}
$$

Based on the above observation, the atmospheric neutrino oscillation amplitudes (2.14) can be further simplified,

$$
\begin{aligned}
S_{\mathrm{ee}} & =S_{11}^{\prime}, \\
S_{\mathrm{e} \mu} & =c_{\mathrm{a}} S_{12}^{\prime}+s_{\mathrm{a}} e^{-i \delta} S_{13}^{\prime}, \\
S_{\mu \mathrm{e}} & =c_{\mathrm{a}} S_{12}^{\prime}+s_{\mathrm{a}} e^{+i \delta} S_{13}^{\prime}, \\
S_{\mu \mu} & =c_{\mathrm{a}}^{2} S_{22}^{\prime}+s_{\mathrm{a}}^{2} S_{33}^{\prime}+2 c_{\mathrm{a}} s_{\mathrm{a}} \cos \delta S_{23}^{\prime} .
\end{aligned}
$$

As a convention, we adopt those elements $S_{\mathrm{ij}}^{\prime}$ with $i \leq j$. It is now manifest that the flavor oscillation amplitudes $S_{\mathrm{e} \mu}$ and $S_{\mu \mathrm{e}}$ differ only by the CP phase and the expression for $S_{\mu \mu}$ (2.14d) is greatly simplified in (2.20d). The oscillation probabilities now read,

$$
\begin{aligned}
P_{\mathrm{ee}} & \equiv\left|S_{\mathrm{ee}}\right|^{2}=\left|S_{11}^{\prime}\right|^{2}, \\
P_{\mathrm{e} \mu} & \equiv\left|S_{\mu \mathrm{e}}\right|^{2}=c_{\mathrm{a}}^{2}\left|S_{12}^{\prime}\right|^{2}+s_{\mathrm{a}}^{2}\left|S_{13}^{\prime}\right|^{2}+2 c_{\mathrm{a}} s_{\mathrm{a}}(\cos \delta \mathbb{R}+\sin \delta \mathbb{I})\left(S_{12}^{\prime} S_{13}^{\prime *}\right), \\
P_{\mu \mathrm{e}} & \equiv\left|S_{\mathrm{e} \mu}\right|^{2}=c_{\mathrm{a}}^{2}\left|S_{12}^{\prime}\right|^{2}+s_{\mathrm{a}}^{2}\left|S_{13}^{\prime}\right|^{2}+2 c_{\mathrm{a}} s_{\mathrm{a}}(\cos \delta \mathbb{R}-\sin \delta \mathbb{I})\left(S_{12}^{\prime} S_{13}^{\prime *}\right), \\
P_{\mu \mu} & \equiv\left|S_{\mu \mu}\right|^{2}=\left|c_{\mathrm{a}}^{2} S_{22}^{\prime}+s_{\mathrm{a}}^{2} S_{33}^{\prime}\right|^{2}+4 c_{\mathrm{a}}^{2} s_{\mathrm{a}}^{2} \cos ^{2} \delta\left|S_{23}^{\prime}\right|^{2}+4 c_{\mathrm{a}} s_{\mathrm{a}} \cos \delta \mathbb{R}\left[\left(c_{\mathrm{a}}^{2} S_{22}^{\prime}+s_{\mathrm{a}}^{2} S_{33}^{\prime}\right) S_{23}^{\prime *}\right] .
\end{aligned}
$$

Throughout our studies in this report we adopt the expression (2.21) for computing the oscillation probabilities in our numerical calculation, which are exact in the limit of the symmetric earth matter profile PREM [98] and neglecting the depth of the detector beneath the earth surface as compared to the baseline lengths. The oscillation probabilities for antineutrinos $\bar{P}_{\alpha \beta}$ are then computed as in (2.18). 


\subsection{Expansion of oscillation probabilities with respect to $x_{\mathrm{a}}=\cos 2 \theta_{\mathrm{a}}$ and $\delta m_{\mathrm{s}}^{2}$}

Although the expressions (2.21) for the oscillation probabilities $P_{\alpha \beta}$, and $\bar{P}_{\alpha \beta}$ via (2.18), are simple enough to perform numerical analysis efficiently, we can obtain further insight by keeping only the leading terms of the following two small parameter of the three neutrino model,

$$
\begin{aligned}
x_{\mathrm{a}} & \equiv \cos 2 \theta_{\mathrm{a}}=\sqrt{1-\sin ^{2} 2 \theta_{\mathrm{a}}}=0.21_{-0.10}^{+0.06}, \\
\frac{\delta m_{\mathrm{s}}^{2}}{\left|\delta m_{\mathrm{a}}^{2}\right|} & =0.032 \pm 0.002,
\end{aligned}
$$

whose numerical values are constrained from the data $[109,111,112]$, as summarized below in (4.3).

First, by expanding $c_{\mathrm{a}}$ and $s_{\mathrm{a}}$ in terms of $x_{\mathrm{a}}$,

$$
c_{\mathrm{a}}^{2}=\frac{1}{2}\left(1+x_{\mathrm{a}}\right), \quad s_{\mathrm{a}}^{2}=\frac{1}{2}\left(1-x_{\mathrm{a}}\right), \quad c_{\mathrm{a}}^{2} s_{\mathrm{a}}^{2}=\frac{1}{4}\left(1-x_{\mathrm{a}}^{2}\right),
$$

the oscillation probabilities $P_{\alpha \beta}(2.21)$ are expanded as,

$$
\begin{aligned}
P_{\mathrm{ee}}= & \left|S_{11}^{\prime}\right|^{2}, \\
P_{\mathrm{e} \mu}= & \frac{1}{2}\left(1-\left|S_{11}^{\prime}\right|^{2}\right)+\frac{x_{\mathrm{a}}}{2}\left(\left|S_{12}^{\prime}\right|^{2}-\left|S_{13}^{\prime}\right|^{2}\right)+\left(\cos \delta^{\prime} \mathbb{R}+\sin \delta^{\prime} \mathbb{I}\right)\left(S_{12}^{\prime} S_{13}^{\prime *}\right)+\mathcal{O}\left(x_{\mathrm{a}}^{4}\right), \\
P_{\mu \mathrm{e}}= & \frac{1}{2}\left(1-\left|S_{11}^{\prime}\right|^{2}\right)+\frac{x_{\mathrm{a}}}{2}\left(\left|S_{12}^{\prime}\right|^{2}-\left|S_{13}^{\prime}\right|^{2}\right)+\left(\cos \delta^{\prime} \mathbb{R}-\sin \delta^{\prime} \mathbb{I}\right)\left(S_{12}^{\prime} S_{13}^{\prime *}\right)+\mathcal{O}\left(x_{\mathrm{a}}^{4}\right), \\
P_{\mu \mu}= & \frac{1}{4}\left|S_{22}^{\prime}+S_{33}^{\prime}\right|^{2}+\frac{x_{\mathrm{a}}}{2}\left(\left|S_{22}^{\prime}\right|^{2}-\left|S_{33}^{\prime}\right|^{2}\right)+\cos \delta^{\prime} \mathbb{R}\left[\left(S_{22}^{\prime}+S_{33}^{\prime}\right) S_{23}^{\prime *}\right] \\
& +x_{\mathrm{a}} \cos \delta^{\prime} \mathbb{R}\left[S_{23}^{\prime}\left(S_{22}^{\prime}-S_{33}^{\prime}\right)^{*}\right]+\frac{1}{4}\left|S_{22}^{\prime}-S_{33}^{\prime}\right|^{2} x_{\mathrm{a}}^{2}+\cos ^{2} \delta^{\prime}\left|S_{23}^{\prime}\right|^{2}+\mathcal{O}\left(x_{\mathrm{a}}^{4}\right) .
\end{aligned}
$$

We can clearly identify the linear terms of $x_{\mathrm{a}}$ in $P_{\mathrm{e} \mu}$ and $P_{\mu \mathrm{e}}$, which are identical, and also in $P_{\mu \mu}$. In the above expansion, we keep the terms of order $x_{\mathrm{a}}^{2}$, which turn out to have significant impacts in the measurement of $x_{a}$ despite the smallness of $x_{\mathrm{a}}^{2} \lesssim 0.05$ at $90 \%$ confidence level. Furthermore, we introduce a short-hand notation,

$$
\cos \delta^{\prime} \equiv 2 c_{\mathrm{a}} s_{\mathrm{a}} \cos \delta \approx \sqrt{1-x_{\mathrm{a}}^{2}} \cos \delta, \quad \sin \delta^{\prime} \equiv 2 c_{\mathrm{a}} s_{\mathrm{a}} \sin \delta \approx \sqrt{1-x_{\mathrm{a}}^{2}} \sin \delta .
$$

in (2.24), without expanding the factor $\sqrt{1-x_{\mathrm{a}}^{2}}$, since all the $\delta$-dependence in the transition probabilities (2.21) are functions of $2 c_{\mathrm{a}} s_{\mathrm{a}} \cos \delta$ and $2 c_{\mathrm{a}} s_{\mathrm{a}} \sin \delta$. The uncertainty of the $\delta$ measurement should be modulated by the factor $1 / \sqrt{1-x_{\mathrm{a}}^{2}}$.

We find it quite useful to express the oscillation probabilities $P_{\alpha \beta}$ in (2.24) and the corresponding antineutrino oscillation probabilities $\bar{P}_{\alpha \beta}$ as,

$$
\begin{gathered}
P_{\alpha \beta} \equiv P_{\alpha \beta}^{(0)}+P_{\alpha \beta}^{(1)} x_{\mathrm{a}}+P_{\alpha \beta}^{(2)} \cos \delta^{\prime}+P_{\alpha \beta}^{(3)} \sin \delta^{\prime}+P_{\alpha \beta}^{(4)} x_{\mathrm{a}} \cos \delta^{\prime}+P_{\alpha \beta}^{(5)} x_{\mathrm{a}}^{2}+P_{\alpha \beta}^{(6)} \cos ^{2} \delta^{\prime}, \\
\bar{P}_{\alpha \beta} \equiv \bar{P}_{\alpha \beta}^{(0)}+\bar{P}_{\alpha \beta}^{(1)} x_{\mathrm{a}}+\bar{P}_{\alpha \beta}^{(2)} \cos \delta^{\prime}+\bar{P}_{\alpha \beta}^{(3)} \sin \delta^{\prime}+\bar{P}_{\alpha \beta}^{(4)} x_{\mathrm{a}} \cos \delta^{\prime}+\bar{P}_{\alpha \beta}^{(5)} x_{\mathrm{a}}^{2}+\bar{P}_{\alpha \beta}^{(6)} \cos ^{2} \delta^{\prime},
\end{gathered}
$$


where $P_{\alpha \beta}^{(0)}$ and $\bar{P}_{\alpha \beta}^{(0)}$ are the leading terms, while $P_{\alpha \beta}^{(k)}$ and $\bar{P}_{\alpha \beta}^{(k)}$ with $k=1, \cdots, 6$ are the coefficients of corresponding terms linear in $x_{\mathrm{a}}, \cos \delta^{\prime}, \sin \delta^{\prime}, x_{\mathrm{a}} \cos \delta^{\prime}, x_{\mathrm{a}}^{2}$, and $\cos ^{2} \delta^{\prime}$, respectively. The magnitude of these coefficients determines the experimental sensitivity of measuring the two mixing parameters, $x_{\mathrm{a}}$ and $\delta$. The coefficients $P_{\alpha \beta}^{(k)}$ of (2.24) are shown in the following table.

\begin{tabular}{c|cccc} 
& $P_{\mathrm{ee}}^{(k)}$ & $P_{\mathrm{e} \mu}^{(k)}$ & $P_{\mu \mathrm{e}}^{(k)}$ & $P_{\mu \mu}^{(k)}$ \\
\hline$(k=0)$ & $\left|S_{11}^{\prime}\right|^{2}$ & $\frac{1}{2}\left(1-\left|S_{11}^{\prime}\right|^{2}\right)$ & $\frac{1}{2}\left(1-\left|S_{11}^{\prime}\right|^{2}\right)$ & $\frac{1}{4}\left|S_{22}^{\prime}+S_{33}^{\prime}\right|^{2}$ \\
$(k=1)$ & 0 & $\frac{1}{2}\left(\left|S_{12}^{\prime}\right|^{2}-\left|S_{13}^{\prime}\right|^{2}\right)$ & $\frac{1}{2}\left(\left|S_{12}^{\prime}\right|^{2}-\left|S_{13}^{\prime}\right|^{2}\right)$ & $\frac{1}{2}\left(\left|S_{22}^{\prime}\right|^{2}-\left|S_{33}^{\prime}\right|^{2}\right)$ \\
$(k=2)$ & 0 & $\mathbb{R}\left(S_{12}^{\prime} S_{13}^{\prime *}\right)$ & $\mathbb{R}\left(S_{12}^{\prime} S_{13}^{\prime *}\right)$ & $\mathbb{R}\left[S_{23}^{\prime}\left(S_{22}^{\prime}+S_{33}^{\prime}\right)^{*}\right]$ \\
$(k=3)$ & 0 & $\mathbb{I}\left(S_{12}^{\prime} S_{13}^{\prime *}\right)$ & $-\mathbb{I}\left(S_{12}^{\prime} S_{13}^{\prime *}\right)$ & 0 \\
$(k=4)$ & 0 & 0 & 0 & $\mathbb{R}\left[S_{23}^{\prime}\left(S_{22}^{\prime}-S_{33}^{\prime}\right)^{*}\right]$ \\
$(k=5)$ & 0 & 0 & 0 & $\frac{1}{4}\left|S_{22}^{\prime}-S_{33}^{\prime}\right|^{2}$ \\
$(k=6)$ & 0 & 0 & 0 & $\left|S_{23}^{\prime}\right|^{2}$
\end{tabular}

It is clearly seen from (2.27) that $P_{\mathrm{ee}}=P\left(\nu_{\mathrm{e}} \rightarrow \nu_{\mathrm{e}}\right)$ has no dependence on $\theta_{\mathrm{a}}$ and $\delta$, all the other oscillation probabilities have terms $P_{\alpha \beta}^{(1)}$ linear in $x_{\mathrm{a}}$, the coefficients of $\cos \delta^{\prime}$ are the same for $P_{\mathrm{e} \mu}$ and $P_{\mu \mathrm{e}}$, those of $\sin \delta^{\prime}$ have the same magnitude but the opposite sign between $P_{\mathrm{e} \mu}$ and $P_{\mu \mathrm{e}}$, while $P_{\mu \mu}$ has no dependence on $\sin \delta^{\prime}$. Most of these properties of the oscillation probabilities are expected from theoretical considerations, while they are made explicit in (2.26) and (2.27). The corresponding coefficients for the antineutrino oscillations, $\bar{P}_{\alpha \beta}^{(k)}$ in $(2.26 \mathrm{~b})$ are obtained from $P_{\alpha \beta}^{(1)}$ in $(2.27)$ as follows:

$$
\begin{aligned}
& \bar{P}_{\alpha \beta}^{(k)}=+P_{\alpha \beta}^{(k)}\left(S_{\mathrm{ij}}^{\prime} \rightarrow \bar{S}_{\mathrm{ij}}^{\prime}\right) \quad \text { for } k=0,1,2,4,5,6, \\
& \bar{P}_{\alpha \beta}^{(3)}=-P_{\alpha \beta}^{(3)}\left(S_{\mathrm{ij}}^{\prime} \rightarrow \bar{S}_{\mathrm{ij}}^{\prime}\right),
\end{aligned}
$$

where $\bar{S}_{\mathrm{ij}}^{\prime}$ are the oscillation amplitudes in the propagation basis which are obtained from $S_{\mathrm{ij}}^{\prime}$ by reversing the sign of the matter potential $a(x)$,

$$
\bar{S}_{\mathrm{ij}}^{\prime}=S_{\mathrm{ij}}^{\prime}(a(x) \rightarrow-a(x))
$$

The relation (2.18) between the $\nu$ and $\bar{\nu}$ oscillation probabilities, $P_{\alpha \beta}$ and $\bar{P}_{\alpha \beta}$, respectively, is simplified significantly in the propagation basis where the matter dependence and the $\delta$ dependence of the oscillation amplitudes are factorized.

The parameter dependences of the oscillation probabilities $P_{\alpha \beta}$ and $\bar{P}_{\alpha \beta}$ are further simplified significantly when we take account of the smallness of the mass squared difference $\delta m_{\mathrm{s}}^{2}$ as compared to $\left|\delta m_{\mathrm{a}}^{2}\right|,(2.22 \mathrm{~b})$. We note in the propagation basis Hamiltonian (2.8) that if we set $\delta m_{\mathrm{s}}^{2} \equiv \delta m_{12}^{2}=0$, then the oscillation occurs only between $\nu_{1}^{\prime}$ and $\nu_{3}^{\prime}$, and hence the transitions between $\nu_{1}^{\prime}$ and $\nu_{2}^{\prime}$, and those between $\nu_{2}^{\prime}$ and $\nu_{3}^{\prime}$ should be suppressed,

$$
\left|S_{12}^{\prime}\right|, \quad\left|S_{23}^{\prime}\right|=\mathcal{O}\left(\frac{\delta m_{\mathrm{s}}^{2}}{\delta m_{\mathrm{a}}^{2}}\right)
$$


in the propagation basis. In our numerical study of the atmospheric neutrino oscillations in the energy range $2 \mathrm{GeV}<E_{\nu}<20 \mathrm{GeV}$, we find $\left|S_{12}^{\prime}\right|<0.15$, and $\left|S_{23}^{\prime}\right|<0.06$. We therefore obtain the following approximation by dropping all the terms of order $\left(\delta m_{\mathrm{s}}^{2} / \delta m_{\mathrm{a}}^{2}\right)^{2}$,

$$
\begin{aligned}
P_{\mathrm{ee}}= & \left|S_{11}^{\prime}\right|^{2} \\
P_{\mathrm{e} \mu}= & \frac{1-x_{\mathrm{a}}}{2}\left(1-\left|S_{11}^{\prime}\right|^{2}\right)+\left(\cos \delta^{\prime} \mathbb{R}+\sin \delta^{\prime} \mathbb{I}\right)\left(S_{12}^{\prime} S_{13}^{\prime *}\right)+\mathcal{O}\left(x_{\mathrm{a}}^{4},\left(\frac{\delta m_{\mathrm{s}}^{2}}{\delta m_{\mathrm{a}}^{2}}\right)^{2}\right), \\
P_{\mu \mathrm{e}}= & \frac{1-x_{\mathrm{a}}}{2}\left(1-\left|S_{11}^{\prime}\right|^{2}\right)+\left(\cos \delta^{\prime} \mathbb{R}-\sin \delta^{\prime} \mathbb{I}\right)\left(S_{12}^{\prime} S_{13}^{\prime *}\right)+\mathcal{O}\left(x_{\mathrm{a}}^{4},\left(\frac{\delta m_{\mathrm{s}}^{2}}{\delta m_{\mathrm{a}}^{2}}\right)^{2}\right), \\
P_{\mu \mu}= & \frac{1}{4}\left|S_{22}^{\prime}+S_{33}^{\prime}\right|^{2}+\frac{x_{\mathrm{a}}}{2}\left(1-\left|S_{11}^{\prime}\right|^{2}\right)+\frac{1}{4} x_{\mathrm{a}}^{2}\left|S_{22}^{\prime}-S_{33}^{\prime}\right|^{2} \\
& -\cos \delta^{\prime} \mathbb{R}\left(S_{12}^{\prime} S_{13}^{\prime *}\right)+x_{\mathrm{a}} \cos \delta^{\prime} \mathbb{R}\left[S_{23}^{\prime}\left(S_{22}^{\prime}-S_{33}^{\prime}\right)^{*}\right]+\mathcal{O}\left(x_{\mathrm{a}}^{4},\left(\frac{\delta m_{\mathrm{s}}^{2}}{\delta m_{\mathrm{a}}^{2}}\right)^{2}\right),
\end{aligned}
$$

and (2.27) is further simplified as follows,

\begin{tabular}{c|cccc} 
& $P_{\mathrm{ee}}^{(k)}$ & $P_{\mathrm{e} \mu}^{(k)}$ & $P_{\mu \mathrm{e}}^{(k)}$ & $P_{\mu \mu}^{(k)}$ \\
\hline$(k=0)$ & $\left|S_{11}^{\prime}\right|^{2}$ & $\frac{1}{2}\left(1-\left|S_{11}^{\prime}\right|^{2}\right)$ & $\frac{1}{2}\left(1-\left|S_{11}^{\prime}\right|^{2}\right)$ & $\frac{1}{4}\left|S_{22}^{\prime}+S_{33}^{\prime}\right|^{2}$ \\
$(k=1)$ & 0 & $-\frac{1}{2}\left(1-\left|S_{11}^{\prime}\right|^{2}\right)$ & $-\frac{1}{2}\left(1-\left|S_{11}^{\prime}\right|^{2}\right)$ & $\frac{1}{2}\left(1-\left|S_{11}^{\prime}\right|^{2}\right)$ \\
$(k=2)$ & 0 & $\mathbb{R}\left(S_{12}^{\prime} S_{13}^{\prime *}\right)$ & $\mathbb{R}\left(S_{12}^{\prime} S_{13}^{\prime *}\right)$ & $-\mathbb{R}\left(S_{12}^{\prime} S_{13}^{\prime *}\right)$ \\
$(k=3)$ & 0 & $\mathbb{I}\left(S_{12}^{\prime} S_{13}^{*}\right)$ & $-\mathbb{I}\left(S_{12}^{\prime} S_{13}^{*}\right)$ & 0 \\
$(k=4)$ & 0 & 0 & 0 & $\mathbb{R}\left[S_{23}^{\prime}\left(S_{22}^{\prime}-S_{33}^{\prime}\right)^{*}\right]$ \\
$(k=5)$ & 0 & 0 & 0 & $\frac{1}{4}\left|S_{22}^{\prime}-S_{33}^{\prime}\right|^{2}$ \\
$(k=6)$ & 0 & 0 & 0 & 0
\end{tabular}

In this approximation, there are only 6 independent oscillation factors in the propagation basis, of which $\left|S_{11}^{\prime}\right|^{2}$ determines the overall rates $P_{\mathrm{ee}}^{(0)}, P_{\mathrm{e} \mu}^{(0)}, P_{\mu \mathrm{e}}^{(0)}$, as well as all the coefficients of $x_{\mathrm{a}}, P_{\mathrm{e} \mu}^{(1)}, P_{\mu \mathrm{e}}^{(1)}$, and $P_{\mu \mu}^{(1)}$. The overall rate $P_{\mu \mu}^{(0)}$ is governed by $\left|S_{22}^{\prime}+S_{23}^{\prime}\right|^{2}$. The coefficients of all the $\sin \delta^{\prime}$ terms are $P_{\mathrm{e} \mu}^{(3)}=-P_{\mu \mathrm{e}}^{(3)}=\mathbb{I}\left(S_{12}^{\prime} S_{13}^{\prime *}\right)$, and the coefficients of the $\cos \delta^{\prime}$ terms are $P_{\mathrm{e} \mu}^{(2)}=P_{\mu \mathrm{e}}^{(2)}=-P_{\mu \mu}^{(2)}=\mathbb{R}\left(S_{12}^{\prime} S_{13}^{\prime *}\right)$. The remaining cross term $x_{\mathrm{a}} \cos \delta^{\prime}$ is governed by $\mathbb{R}\left[S_{23}^{\prime}\left(S_{22}^{\prime}-S_{33}^{\prime}\right)^{*}\right]$ in $P_{\mu \mu}^{(4)}$. For the $x_{\mathrm{a}}^{2}$ term, its coefficient $\left|S_{22}^{\prime}-S_{33}^{\prime}\right|^{2}$ is also independent. The coefficient $P_{\mu \mu}^{(6)}$ of $\cos ^{2} \delta^{\prime}$ in $(2.26)$ and $(2.27)$ is $\left(\delta m_{s}^{2} / \delta m_{a}^{2}\right)^{2}$ order, and hence is dropped in (2.31) and (2.32).

We examine the energy and zenith angle dependence of these 6 oscillation factors in figure 1 and figure 2. The amplitude matrix elements $S_{i j}^{\prime}$ in the propagation basis can be calculated numerically in the way described in section A.3. Shown in figure 1(a) and (b) are the $E_{\nu}$ dependence of the coefficients $P_{\mathrm{ee}}^{(0)}=\left|S_{11}^{\prime}\right|^{2}\left(\bar{P}_{\mathrm{ee}}^{(0)}=\left|\bar{S}_{11}^{\prime}\right|^{2}\right)$ and $P_{\mu \mu}^{(0)}=$ $\left|S_{22}^{\prime}+S_{33}^{\prime}\right|^{2} / 4\left(\bar{P}_{\mu \mu}^{(0)}=\left|\bar{S}_{22}^{\prime}+\bar{S}_{33}^{\prime}\right|^{2}\right)$, respectively, for the baseline along five zenith angles, $\cos \theta_{\mathrm{z}}=-1,-0.9,-0.8,-0.6$ and -0.4 . In each panel, the solid and dashed curves are for $\nu$ and $\bar{\nu}$ oscillations, respectively, shown by the thick lines for $\mathrm{NH}$ and by the thin lines for IH. It should be noted that the coefficient $\left|S_{11}^{\prime}\right|$ in figure 1 not only determines 

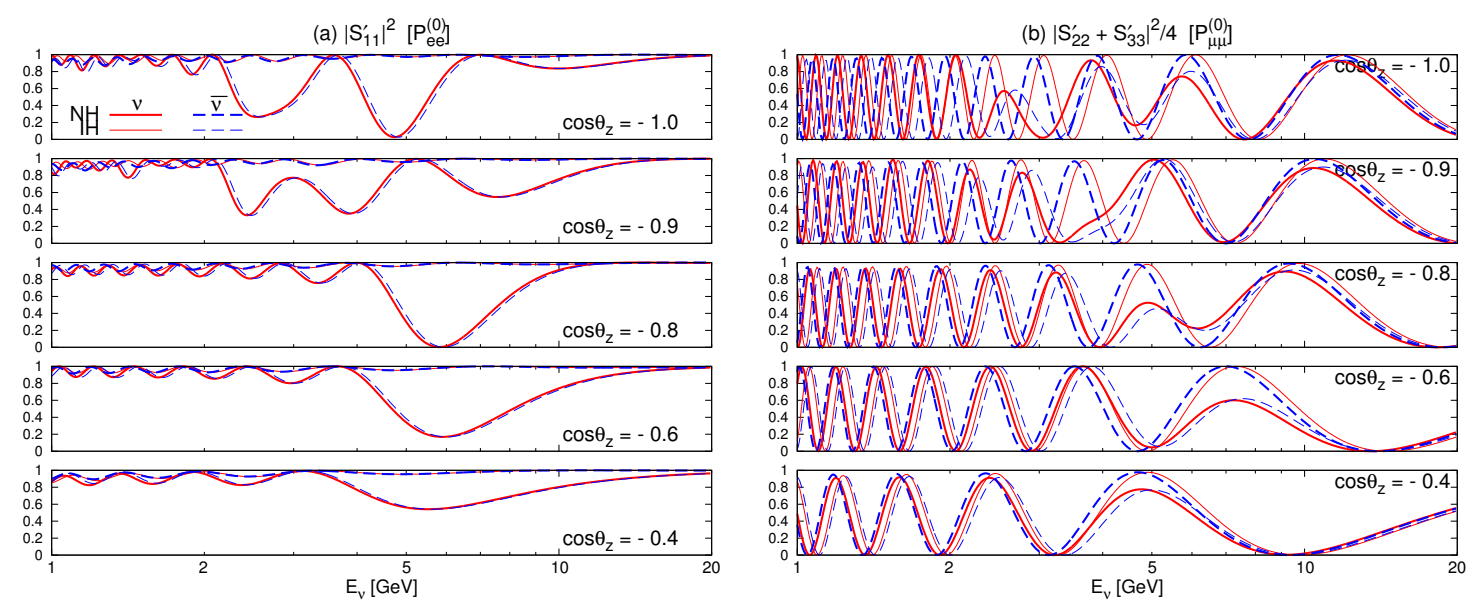

Figure 1. (a) The oscillation probabilities $P_{\mathrm{ee}}^{(0)}=\left|S_{11}^{\prime}\right|^{2}$ [solid lines], and $\bar{P}_{\mathrm{ee}}^{(0)}=\left|\bar{S}_{11}^{\prime}\right|^{2}$ [dashed lines] plotted against the $\nu$ and $\bar{\nu}$ energies, respectively, for the zenith angles $\cos \theta_{\mathrm{z}}=$ $-1,-0.9,-0.8,-0.6,-0.4$. The thick curves are for NH, while the thin curves are for IH. (b) The same as (a), but for the coefficient $P_{\mu \mu}^{(0)}(\nu)$ and $\bar{P}_{\mu \mu}^{(0)}(\bar{\nu})$.

$P_{\mathrm{ee}}^{(0)}$, as in $(2.16 \mathrm{a})$, but also governs all the coefficients of $x_{\mathrm{a}}, P_{\mathrm{e} \mu}^{(1)}=P_{\mu \mathrm{e}}^{(1)}=P_{\mu \mu}^{(1)}$ in the approximation (2.31).

We immediately notice in figure 1 (a) the absence of the significant oscillation in $P_{\mathrm{ee}}^{(0)}$ for the $\nu$ in $\mathrm{IH}$ [solid-thin lines] and for the $\bar{\nu}\left(\bar{P}_{\mathrm{ee}}^{(0)}\right)$ in $\mathrm{NH}$ [dashed-thick lines]. Likewise, in figure 1(b) for $P_{\mu \mu}^{(0)}(\mathrm{IH})$ and $\bar{P}_{\mu \mu}^{(0)}(\mathrm{NH})$, the oscillation curves for the same contributions, solid-thin and dashed-thick lines, show the vacuum-oscillation like pattern. They are consequences of the absence of the MSW resonance in these cases, as explained in section A.4. Conversely, the strong oscillation pattern for $P_{\text {ee }}(\mathrm{NH})$ and $\bar{P}_{\text {ee }}(\mathrm{IH})$ in figure $1(\mathrm{a})$ and the significant deviation from the vacuum oscillation pattern for $P_{\mu \mu}^{(0)}(\mathrm{NH})$ and $\bar{P}_{\mu \mu}^{(0)}(\mathrm{IH})$ in figure 1(b) are both consequences of the MSW resonance at $E_{\nu} \sim 6 \mathrm{GeV}$ for the earth matter density of $\rho \sim 5 \mathrm{~g} / \mathrm{cm}^{2}$ along the baseline with $\cos \theta_{\mathrm{z}}<-0.6$; see figure 12. More generally, we find,

$$
\begin{array}{ll}
\bar{P}_{\alpha \beta}^{(k)}(\mathrm{NH}) \approx+P_{\alpha \beta}^{(k)}(\mathrm{IH}) \quad \text { for } \quad k=0,1,3,5,6, \\
\bar{P}_{\alpha \beta}^{(k)}(\mathrm{NH}) \approx-P_{\alpha \beta}^{(k)}(\mathrm{IH}) \quad \text { for } \quad k=2,4,
\end{array}
$$

and vice versa for $\bar{P}_{\alpha \beta}^{(k)}(\mathrm{IH})$. The relative minus signs for $k=2,3,4$, as compared to the relations (2.28) within the same hierarchy, are consequences of the extra minus sign in $S_{13}^{\prime}$ and $S_{22}^{\prime}-S_{33}^{\prime}$, when both $\delta m_{\mathrm{a}}^{2}$ and $a(x)$ reverse signs in the limit of vanishing $\delta m_{\mathrm{s}}^{2}$.

Therefore, if we observe the presence or absence of the MSW resonance effects in $\nu$ and $\bar{\nu}$ oscillations, we can determine the neutrino mass hierarchy. However, as shown clearly in figures $1(\mathrm{a})$ and (b), the oscillation probabilities of $\nu$ in one mass hierarchy are very similar to those of $\bar{\nu}$ in the other mass hierarchy. Therefore, the capability of an atmospheric neutrino detector that cannot distinguish particle charges depend critically on the difference in the flux times cross section products of $\nu$ and $\bar{\nu}$, as shown in figure 8.

In figure 2 , we show the coefficients of $\cos \delta^{\prime}=\sqrt{1-x_{\mathrm{a}}^{2}} \cos \delta$ and $\sin \delta^{\prime}=\sqrt{1-x_{\mathrm{a}}^{2}} \sin \delta$, which determines the sensitivity of the neutrino oscillation among the $e$ and $\mu$ flavors on 

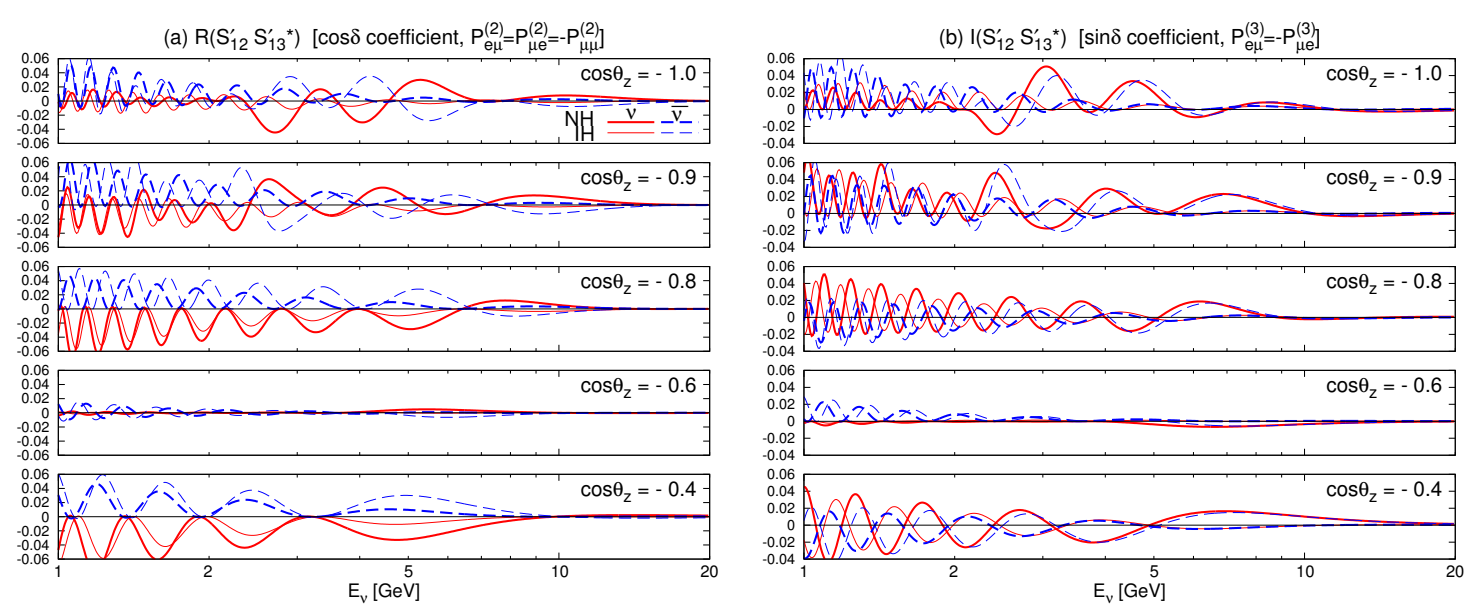

Figure 2. (a) The coefficients of $\cos \delta^{\prime}=\sqrt{1-x_{\mathrm{a}}^{2}} \cos \delta, \mathbb{R}\left(S_{12}^{\prime} S_{13}^{\prime *}\right)=P_{\mathrm{e} \mu}^{(2)}=P_{\mu \mathrm{e}}^{(2)}=-P_{\mu \mu}^{(2)}$ and $\mathbb{R}\left(\bar{S}_{12}^{\prime} \bar{S}_{13}^{\prime *}\right)=\bar{P}_{\mathrm{e} \mu}^{(2)}=\bar{P}_{\mu \mathrm{e}}^{(2)}=-\bar{P}_{\mu \mu}^{(2)}$, and (b) the coefficients of $\sin \delta^{\prime}=\sqrt{1-x_{\mathrm{a}}^{2}} \sin \delta, \mathbb{I}\left(S_{12}^{\prime} S_{13}^{\prime *}\right)=$ $P_{\mathrm{e} \mu}^{(3)}=-P_{\mu \mathrm{e}}^{(3)}$ and $\mathbb{I}\left(\bar{S}_{12}^{\prime} \bar{S}_{13}^{\prime *}\right)=-\bar{P}_{\mathrm{e} \mu}^{(3)}=\bar{P}_{\mu \mathrm{e}}^{(3)}$, are plotted against the neutrino energy $E_{\nu}$ for $\cos \theta_{\mathrm{z}}=-1,-0.9,-0.8,-0.6,-0.4$. The thick and thin lines are for $\mathrm{NH}$ and $\mathrm{IH}$, respectively, for $\nu$ [solid lines] and $\bar{\nu}$ [dashed lines] oscillations.

the CP phase $\delta$. The real part $\mathbb{R}\left(S_{12}^{\prime} S_{13}^{*}\right)$ shown in figure 2(a) governs all the coefficients of $\cos \delta^{\prime}$, see (2.32), whereas the imaginary part $\mathbb{I}\left(S_{12}^{\prime} S_{13}^{\prime *}\right)$ in figure $2(\mathrm{~b})$ dictates the $\sin \delta^{\prime}$ coefficients of $P_{\mathrm{e} \mu}^{(3)}=-P_{\mu \mathrm{e}}^{(3)}$ and $\bar{P}_{\mathrm{e} \mu}^{(3)}=-\bar{P}_{\mu \mathrm{e}}^{(3)}$. In all the cases, we confirm the vacuum oscillation like patterns for $\nu$ in $\mathrm{IH}$ [solid-thin lines] and for $\bar{\nu}$ in $\mathrm{NH}$ [dashed-thick lines], and significantly different patterns for $\nu$ in $\mathrm{NH}$ [solid-thick lines] and for $\bar{\nu}$ in $\mathrm{IH}$ [dashedthin lines]. The approximate relations (2.33) between the $\nu$ oscillation in $\mathrm{IH}$ and the $\bar{\nu}$ oscillation in $\mathrm{NH}$, and vice versa, between the $\nu$ oscillation in $\mathrm{NH}$ and the $\bar{\nu}$ oscillation in IH also holds rather well, despite small phase-shifts due to oscillations in $\delta m_{\mathrm{s}}^{2}$. In addition, we note the smallness of their magnitudes, typically at the level of $3 \%$ for $\cos \theta_{\mathrm{z}}<-0.8$, being terms of order $\delta m_{\mathrm{s}}^{2} / \delta m_{\mathrm{a}}^{2}$. Note that they are larger at lower energies, $E_{\nu} \lesssim 6 \mathrm{GeV}$. Consequently, the measurement of the CP phase $\delta$ may require sensitivity to the $\nu_{\mu} \leftrightarrow \nu_{\mathrm{e}}$ and $\bar{\nu}_{\mu} \leftrightarrow \bar{\nu}_{\mathrm{e}}$ oscillations at relatively low energies.

Finally, in figure 3(a) we show the coefficients of the cross term $x_{\mathrm{a}} \cos \delta^{\prime}$, which appears only in $P_{\mu \mu}^{(4)}$ and $\bar{P}_{\mu \mu}^{(4)}$, and in figure $3(\mathrm{~b})$ the coefficients $P_{\mu \mu}^{(5)}$ and $\bar{P}_{\mu \mu}^{(5)}$ of the quadratic term $x_{\mathrm{a}}^{2}$. We should note that $P_{\alpha \beta}^{(4)}$ is of the same order as $P_{\alpha \beta}^{(2)}$ and $P_{\alpha \beta}^{(3)}$, and also satisfy the same features described in the last paragraph. Generally speaking, the coefficient of the $x_{\mathrm{a}} \cos \delta$ mixing term is small in magnitude as compared to those of $x_{\mathrm{a}}, P_{\mathrm{e} \mu}^{(1)}=P_{\mu \mathrm{e}}^{(1)}=-P_{\mu \mu}^{(1)}=$ $-\frac{1}{2}\left(1-\left|S_{11}^{\prime}\right|^{2}\right)$ as can be inferred from the $\left|S_{11}^{\prime}\right|^{2}$ plots of figure 1(a), especially at high energies of $E_{\nu} \gtrsim 5 \mathrm{GeV}$. Therefore, we expect little $\delta$-dependence in the $x_{\mathrm{a}}$ measurement. On the other hand, the coefficients of $x_{\mathrm{a}}^{2}, P_{\mu \mu}^{(5)}$ and $\bar{P}_{\mu \mu}^{(5)}$ shown in figure $3(\mathrm{~b})$, are large in magnitudes and can dominate the terms linear in $x_{\mathrm{a}}$, even for $x_{\mathrm{a}}^{2} \sim 0.04$, especially for the neutrino oscillation in $\mathrm{IH}$ and the antineutrino oscillation in NH where $\frac{1}{2}\left(1-\left|S_{11}^{\prime}\right|^{2}\right)$ has very small magnitude at $E_{\nu} \gtrsim 4 \mathrm{GeV}$, see the thin-solid and thick-dashed curves in figure 1(a). Consequently, their contributions can be significant in the measurement of $x_{a}$. 

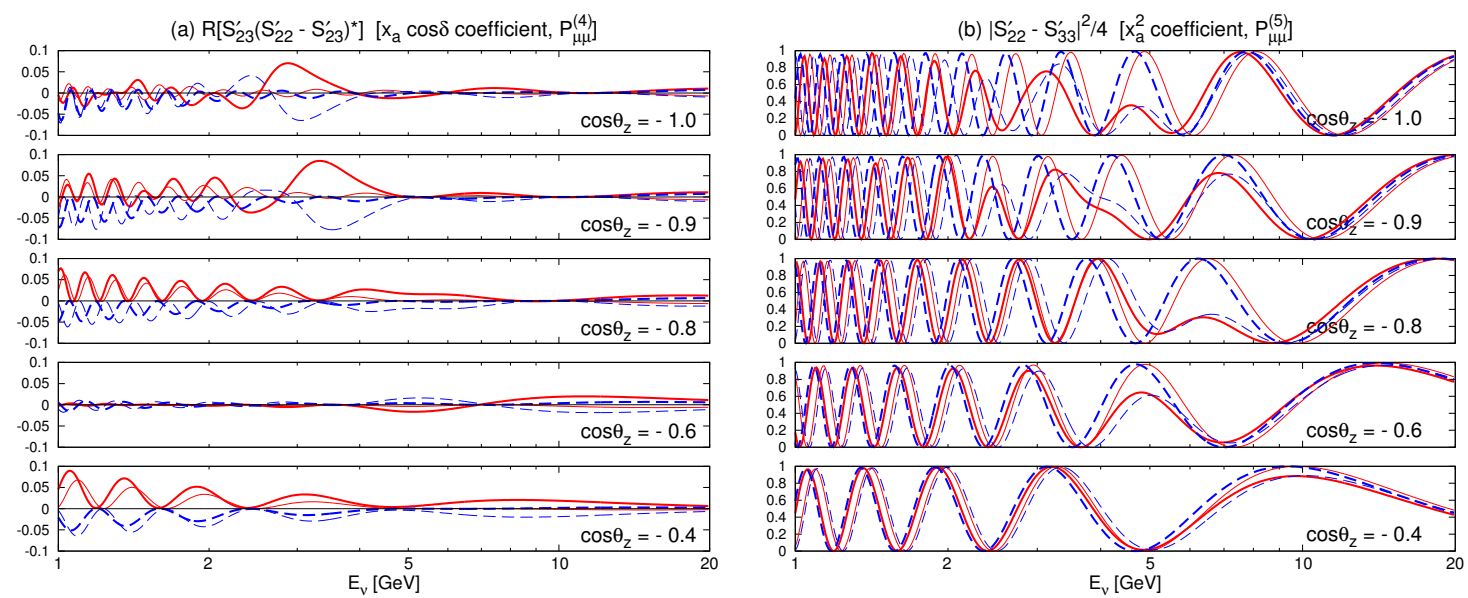

Figure 3. (a) The coefficients $\mathbb{R}\left[S_{23}^{\prime}\left(S_{22}^{\prime}-S_{33}^{\prime}\right)^{*}\right]=P_{\mu \mu}^{(4)}$ and $\mathbb{R}\left[\bar{S}_{23}^{\prime}\left(\bar{S}_{22}^{\prime}-\bar{S}_{33}^{\prime}\right)^{*}\right]=\bar{P}_{\mu \mu}^{(4)}$ of the cross term $x_{\mathrm{a}} \cos \delta^{\prime}=x_{\mathrm{a}} \sqrt{1-x_{\mathrm{a}}^{2}} \cos \delta$, and (b) the coefficients $\frac{1}{4}\left|S_{22}^{\prime}-S_{33}^{\prime}\right|^{2}=P_{\mu \mu}^{(5)}$ and $\frac{1}{4}\left|\bar{S}_{22}^{\prime}-\bar{S}_{33}^{\prime}\right|^{2}=\bar{P}_{\mu \mu}^{(5)}$ of the quadratic term $x_{\mathrm{a}}^{2}$, are plotted against the neutrino energy $E_{\nu}$ for $\cos \theta_{\mathrm{z}}=-1,-0.9,-0.8,-0.6,-0.4$. The thick and thin lines are for $\mathrm{NH}$ and $\mathrm{IH}$, respectively, for $\nu$ [solid lines] and $\bar{\nu}$ [dashed lines] oscillations.

\section{Event rates at a charge-blind detector}

We are now ready to study systematically the event rate distributions of atmospheric neutrino observation at PINGU. It cannot distinguish particle charges, but has the capability of resolving high energy $\mu^{ \pm}$tracks from $e^{ \pm}$and/or hadronic showers [53-55].

Out from the four components in the atmospheric neutrino flux, namely the fluxes of electron- and muon-neutrino/antineutrino, as shown in figure 8, the PINGU detector [57] is assumed to observe both electron-like and muon-like events [58],

$$
\begin{aligned}
& \frac{d N_{\mathrm{e}}}{d E_{\nu} d \cos \theta_{\mathrm{z}}}= \\
& \quad\left\{\left[\phi_{\nu_{\mathrm{e}}}\left(E_{\nu}, \cos \theta_{\mathrm{z}}\right) P_{\mathrm{ee}}\left(E_{\nu}, \cos \theta_{\mathrm{z}}\right)+\phi_{\nu_{\mu}}\left(E_{\nu}, \cos \theta_{\mathrm{z}}\right) P_{\mu \mathrm{e}}\left(E_{\nu}, \cos \theta_{\mathrm{z}}\right)\right] \sigma_{\nu_{\mathrm{e}}}\left(E_{\nu}\right)\right. \\
& \left.\quad+\left[\phi_{\bar{\nu}_{\mathrm{e}}}\left(E_{\nu}, \cos \theta_{\mathrm{z}}\right) \bar{P}_{\mathrm{ee}}\left(E_{\nu}, \cos \theta_{\mathrm{z}}\right)+\phi_{\bar{\nu}_{\mu}}\left(E_{\nu}, \cos \theta_{\mathrm{z}}\right) \bar{P}_{\mu \mathrm{e}}\left(E_{\nu}, \cos \theta_{\mathrm{z}}\right)\right] \sigma_{\bar{\nu}_{\mathrm{e}}}\left(E_{\nu}\right)\right\} \rho V_{\mathrm{eff}}\left(E_{\nu}\right), \\
& \quad d N_{\mu} \\
& \quad \frac{d E_{\nu} d \cos \theta_{\mathrm{z}}}{\quad\{} \\
& \quad+\left[\phi_{\nu_{\mathrm{e}}}\left(E_{\nu}, \cos \theta_{\mathrm{z}}\right) P_{\mathrm{e} \mu}\left(E_{\nu}, \cos \theta_{\mathrm{z}}\right)+\phi_{\nu_{\mu}}\left(E_{\nu}, \cos \theta_{\mathrm{z}}\right) P_{\mu \mu}\left(E_{\nu}, \cos \theta_{\mathrm{z}}\right)\right] \sigma_{\nu_{\mu}}\left(E_{\nu}\right) \\
& \left.\quad+\left[\phi_{\bar{\nu}_{\mathrm{e}}}\left(E_{\nu}, \cos \theta_{\mathrm{z}}\right) \bar{P}_{\mathrm{e} \mu}\left(E_{\nu}, \cos \theta_{\mathrm{z}}\right)+\phi_{\bar{\nu}_{\mu}}\left(E_{\nu}, \cos \theta_{\mathrm{z}}\right) \bar{P}_{\mu \mu}\left(E_{\nu}, \cos \theta_{\mathrm{z}}\right)\right] \sigma_{\bar{\nu}_{\mu}}\left(E_{\nu}\right)\right\} \rho V_{\mathrm{eff}}\left(E_{\nu}\right), \\
& \quad
\end{aligned}
$$

by summing over contributions from charged-current $(\mathrm{CC}) e^{ \pm}$and $\mu^{ \pm}$production events. Since the fiducial volume is universal for both $e^{ \pm}$and $\mu^{ \pm}$channels, as explained in section A.1, it serves as an overall factor. For each flavor, neutrino and antineutrino contribute with the corresponding $\mathrm{CC}$ cross sections. It should be noted that we neglect the contributions from tau-neutrino/antineutrino since the tau-neutrino flux is very small [102] and also because the charged-current $\tau^{ \pm}$production events followed by their pure-leptonic decays contribute mainly to events with low observable energies, which may not contribute much 
due to the smaller fiducial volume. In addition, the tau neutrino cross section is small compared to the CC electron and muon neutrino cross sections [103]. Contributions from the charged-current $\tau^{ \pm}$production events as well as neutral-current events will be studied elsewhere.

Since the number of signal events depends on the oscillation probabilities linearly, which have been decomposed into six terms in (2.26), the event rates can also be decomposed accordingly,

$\frac{d N_{\alpha}}{d E_{\nu} d \cos \theta_{\mathrm{z}}} \equiv N_{\alpha}^{(0)}+N_{\alpha}^{(1)} x_{\mathrm{a}}+N_{\alpha}^{(2)} \cos \delta^{\prime}+N_{\alpha}^{(3)} \sin \delta^{\prime}+N_{\alpha}^{(4)} x_{\mathrm{a}} \cos \delta^{\prime}+N_{\alpha}^{(5)} x_{\mathrm{a}}^{2}+N_{\alpha}^{(6)} \cos ^{2} \delta^{\prime}$.

By combining with the explicit expressions of the decomposed oscillation probabilities in (2.27), the coefficients for electron-like event number rates are,

$$
\begin{aligned}
N_{\mathrm{e}}^{(0)} & =\left\{\left[\phi_{\nu_{\mathrm{e}}}\left|S_{11}^{\prime}\right|^{2}+\phi_{\nu_{\mu}} \frac{1}{2}\left(1-\left|S_{11}^{\prime}\right|^{2}\right)\right] \sigma_{\nu_{\mathrm{e}}}+\left[\phi_{\bar{\nu}_{\mathrm{e}}}\left|\bar{S}_{11}^{\prime}\right|^{2}+\phi_{\bar{\nu}_{\mu}} \frac{1}{2}\left(1-\left|\bar{S}_{11}^{\prime}\right|^{2}\right)\right] \sigma_{\bar{\nu}_{\mathrm{e}}}\right\} \rho V_{\mathrm{eff}} \\
N_{\mathrm{e}}^{(1)} & =\left\{-\phi_{\nu_{\mu}} \frac{1}{2}\left(1-\left|S_{11}^{\prime}\right|^{2}\right) \sigma_{\nu_{\mathrm{e}}}-\phi_{\bar{\nu}_{\mu}} \frac{1}{2}\left(1-\left|\bar{S}_{11}^{\prime}\right|^{2}\right) \sigma_{\bar{\nu}_{\mathrm{e}}}\right\} \rho V_{\mathrm{eff}} \\
N_{\mathrm{e}}^{(2)} & =\left[+\phi_{\nu_{\mu}} \mathbb{R}\left(S_{12}^{\prime} S_{13}^{\prime *}\right) \sigma_{\nu_{\mathrm{e}}}+\phi_{\bar{\nu}_{\mu}} \mathbb{R}\left(\bar{S}_{12}^{\prime} \bar{S}_{13}^{\prime *}\right) \sigma_{\bar{\nu}_{\mathrm{e}}}\right] \rho V_{\mathrm{eff}} \\
N_{\mathrm{e}}^{(3)} & =\left[-\phi_{\nu_{\mu}} \mathbb{I}\left(S_{12}^{\prime} S_{13}^{\prime *}\right) \sigma_{\nu_{\mathrm{e}}}+\phi_{\bar{\nu}_{\mu}} \mathbb{I}\left(\bar{S}_{12}^{\prime} \bar{S}_{13}^{\prime *}\right) \sigma_{\bar{\nu}_{\mathrm{e}}}\right] \rho V_{\mathrm{eff}} \\
N_{\mathrm{e}}^{(4)} & =N_{\mathrm{e}}^{(5)}=N_{\mathrm{e}}^{(6)}=0
\end{aligned}
$$

For brevity, the arguments $E_{\nu}$ and $\cos \theta_{\mathrm{z}}$ have been omitted. Note that there is no term with $x_{\mathrm{a}} \cos \delta^{\prime}, x_{\mathrm{a}}^{2}$ or $\cos ^{2} \delta^{\prime}$ dependence for electron-like events since $P_{\mathrm{ee}}^{(4)}=P_{\mu \mathrm{e}}^{(4)}=P_{\mathrm{ee}}^{(5)}=$ $P_{\mu \mathrm{e}}^{(5)}=P_{\mathrm{ee}}^{(6)}=P_{\mu \mathrm{e}}^{(6)}=0$ and the same for antineutrinos as shown in (2.27) and (2.32). In other words, the atmospheric angle $\theta_{\mathrm{a}}$ and the $\mathrm{CP}$ phase $\delta$ are naturally disentangled in the electron-like events, which depend on $\theta_{\mathrm{a}}$ through $N_{\mathrm{e}}^{(1)}$ while the dependence on the CP phase $\delta$ comes from $N_{\mathrm{e}}^{(2)}$ and $N_{\mathrm{e}}^{(3)}$.

For the muon-like events, we find,

$$
\begin{aligned}
N_{\mu}^{(0)}= & \left\{\left[\phi_{\nu_{\mathrm{e}}} \frac{1}{2}\left(1-\left|S_{11}^{\prime}\right|^{2}\right)+\phi_{\nu_{\mu}} \frac{1}{4}\left|S_{22}^{\prime}+S_{33}^{\prime}\right|^{2}\right] \sigma_{\nu_{\mu}}\right. \\
& \left.+\left[\phi_{\bar{\nu}_{\mathrm{e}}} \frac{1}{2}\left(1-\left|\bar{S}_{11}^{\prime}\right|^{2}\right)+\phi_{\bar{\nu}_{\mu}} \frac{1}{4}\left|\bar{S}_{22}^{\prime}+\bar{S}_{33}^{\prime}\right|^{2}\right] \sigma_{\bar{\nu}_{\mu}}\right\} \rho V_{\mathrm{eff}}, \\
N_{\mu}^{(1)}= & \left\{\left(\phi_{\nu_{\mu}}-\phi_{\nu_{\mathrm{e}}}\right) \frac{1}{2}\left(1-\left|S_{11}^{\prime}\right|^{2}\right) \sigma_{\nu_{\mu}}+\left(\phi_{\bar{\nu}_{\mu}}-\phi_{\bar{\nu}_{\mathrm{e}}}\right) \frac{1}{2}\left(1-\left|\bar{S}_{11}^{\prime}\right|^{2}\right) \sigma_{\bar{\nu}_{\mu}}\right\} \rho V_{\mathrm{eff}}, \\
N_{\mu}^{(2)}= & \left\{\left(\phi_{\nu_{\mathrm{e}}}-\phi_{\nu_{\mu}}\right) \mathbb{R}\left(S_{12}^{\prime} S_{13}^{\prime *}\right) \sigma_{\nu_{\mu}}+\left(\phi_{\bar{\nu}_{\mathrm{e}}}-\phi_{\bar{\nu}_{\mu}}\right) \mathbb{R}\left(\bar{S}_{12}^{\prime} \bar{S}_{13}^{\prime *}\right) \sigma_{\bar{\nu}_{\mu}}\right\} \rho V_{\mathrm{eff}}, \\
N_{\mu}^{(3)}= & \left\{\phi_{\nu_{\mathrm{e}}} \mathbb{I}\left(S_{12}^{\prime} S_{13}^{\prime *}\right) \sigma_{\nu_{\mu}}-\phi_{\bar{\nu}_{\mathrm{e}}} \mathbb{I}\left(\bar{S}_{12}^{\prime} \bar{S}_{13}^{\prime *}\right) \sigma_{\bar{\nu}_{\mu}}\right\} \rho V_{\mathrm{eff}}, \\
N_{\mu}^{(4)}= & \left\{\phi_{\nu_{\mu}} \mathbb{R}\left[S_{23}^{\prime}\left(S_{22}^{\prime}-S_{33}^{\prime}\right)^{*}\right] \sigma_{\nu_{\mu}}+\phi_{\bar{\nu}_{\mu}} \mathbb{R}\left[\bar{S}_{23}^{\prime}\left(\bar{S}_{22}^{\prime}-\bar{S}_{33}^{\prime}\right)^{*}\right] \sigma_{\bar{\nu}_{\mu}}\right\} \rho V_{\mathrm{eff}}, \\
N_{\mu}^{(5)}= & \left\{\phi_{\nu_{\mu}} \frac{1}{4}\left|S_{22}^{\prime}-S_{33}^{\prime}\right|^{2} \sigma_{\nu_{\mu}}+\phi_{\bar{\nu}_{\mu}} \frac{1}{4}\left|\bar{S}_{22}^{\prime}-\bar{S}_{33}^{\prime}\right|^{2} \sigma_{\bar{\nu}_{\mu}}\right\} \rho V_{\mathrm{eff}}, \\
N_{\mu}^{(6)=} & \left\{\phi_{\nu_{\mu}}\left|S_{23}^{\prime}\right|^{2} \sigma_{\nu_{\mu}}+\phi_{\bar{\nu}_{\mu}}\left|\bar{S}_{23}^{\prime}\right|^{2} \sigma_{\bar{\nu}_{\mu}}\right\} \rho V_{\mathrm{eff}} .
\end{aligned}
$$



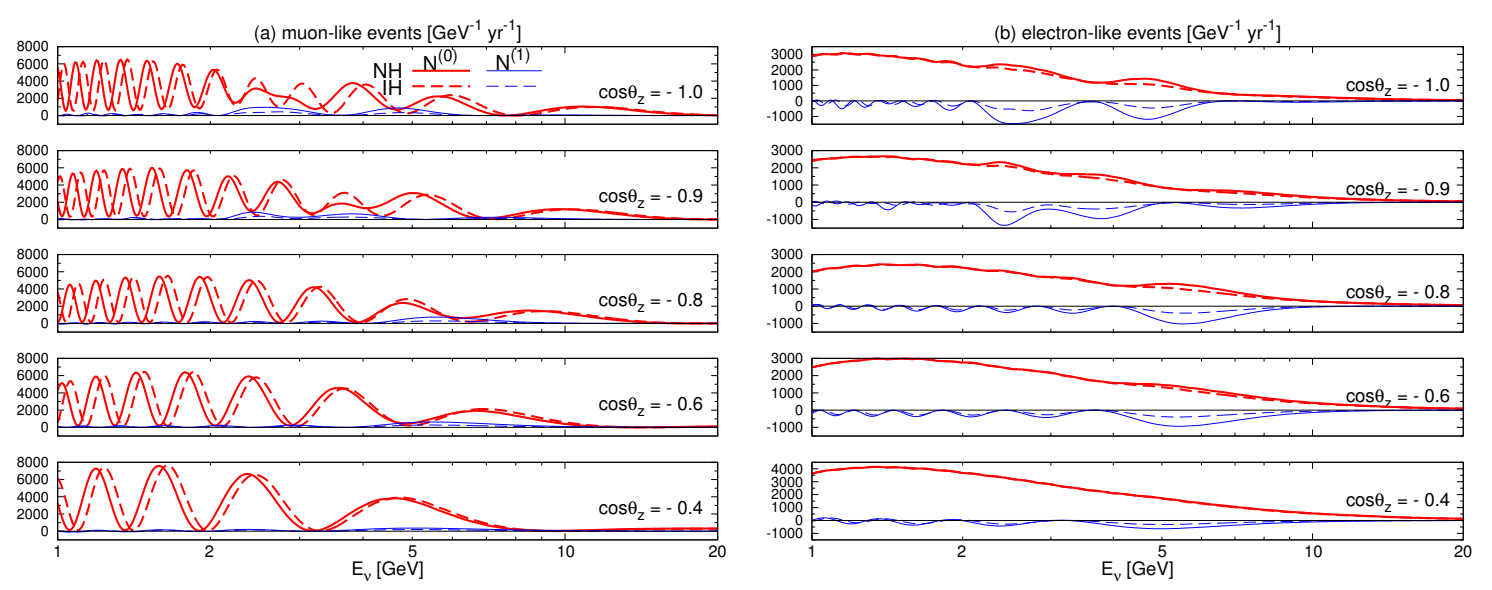

Figure 4. The overall rates $N_{\alpha}^{(0)}$ [red-thick lines] and the coefficients $N_{\alpha}^{(1)}$ of $x_{\mathrm{a}}$ [blue-thin lines] are plotted against $E_{\nu}$ at $\cos \theta_{\mathrm{z}}=-1,-0.9,-0.8,-0.6,-0.4$, for the muon-like $[\alpha=\mu]$ events (a) and the electron-like $[\alpha=e]$ events (b). The solid curves are for NH, while the dashed curves are for IH. The vertical scale gives the number of events per $\mathrm{GeV}$ in one year.

Note that for muon-like events, the crossing term $x_{\mathrm{a}} \cos \delta^{\prime}$ has nonvanishing coefficient $N_{\mu}^{(4)}$. Consequently, with muon-like events included, we should observe some correlation between the measurements of the atmospheric mixing angle $x_{\mathrm{a}}$ and the $\mathrm{CP}$ phase $\delta$, as will be described in section 4 . In addition, a nonzero $N_{\mu}^{(6)}$, which is one further order of magnitude smaller than $N_{\mu}^{(4)}$, is kept according to (2.27) just to show its magnitude.

By combining everything together, the atmospheric neutrino flux, cross section and effective fiducial volume of figure 8 in section A.1, the earth matter profile in section A.2, and the oscillation probabilities discussed in section 2 together with the numerical method described in section A.3, the energy and the zenith angle dependences of the coefficients for muon- and electron-like event rates are shown in figure 4, figure 5, figure 6 , and figure 7 . Note the different scales of the plots, which are adjusted to show the structure of the coefficients.

In figure 4, we show the overall rates $N_{\alpha}^{(0)}$ in red-thick lines and the coefficients $N_{\alpha}^{(1)}$ of $x_{\mathrm{a}}$ in blue-thin lines, as functions of $E_{\nu}$ at several $\cos \theta_{\mathrm{z}}$, for muon-like $[\alpha=\mu]$ events (a) and for electron-like $[\alpha=e]$ events (b). The solid curves are for the normal hierarchy $(\mathrm{NH})$, while the dashed curves are for the inverted hierarchy (IH). The muon-like event rates $N_{\mu}^{(0)}$ in the left panel figure 4(a) show significant oscillatory behavior for both NH (thick-solid lines) and IH (thick-dashed lines). However, the huge hierarchy dependences in the $\nu_{\mu} \rightarrow \nu_{\mu}$ oscillation (MSW [73-76] resonance only for NH) and in the $\bar{\nu}_{\mu} \rightarrow \bar{\nu}_{\mu}$ oscillation (MSW resonance only for IH) as shown in figure 1(b) diminish significantly because of the cancellation between the $\nu_{\mu}$ and $\bar{\nu}_{\mu}$ contributions. Because the flux times cross section for $\nu_{\mu}$ is a factor of about three larger than that for $\bar{\nu}_{\mu}$ as shown in figure 8(b), the hierarchy dependence of the $\nu_{\mu} \rightarrow \nu_{\mu}$ oscillation survives, resulting in the smaller rate for IH at the MSW resonant energy of $\sim 6 \mathrm{GeV}$ at $\cos \theta_{\mathrm{z}}-0.8$. Especially at $\cos \theta_{\mathrm{z}} \lesssim-0.9$, shown in the top two panels of figure 4(a), the nearly maximal resonant oscillation of $\nu_{\mu} \rightarrow \nu_{\mu}$ for $\mathrm{NH}$ at $E_{\nu} \sim 4 \mathrm{GeV}$ shown by the thick-red curves in the top two panels of figure 1 (b) gives rise to the significant difference in the muon-like event rate in the $3 \sim 5 \mathrm{GeV}$ region due to the so- 

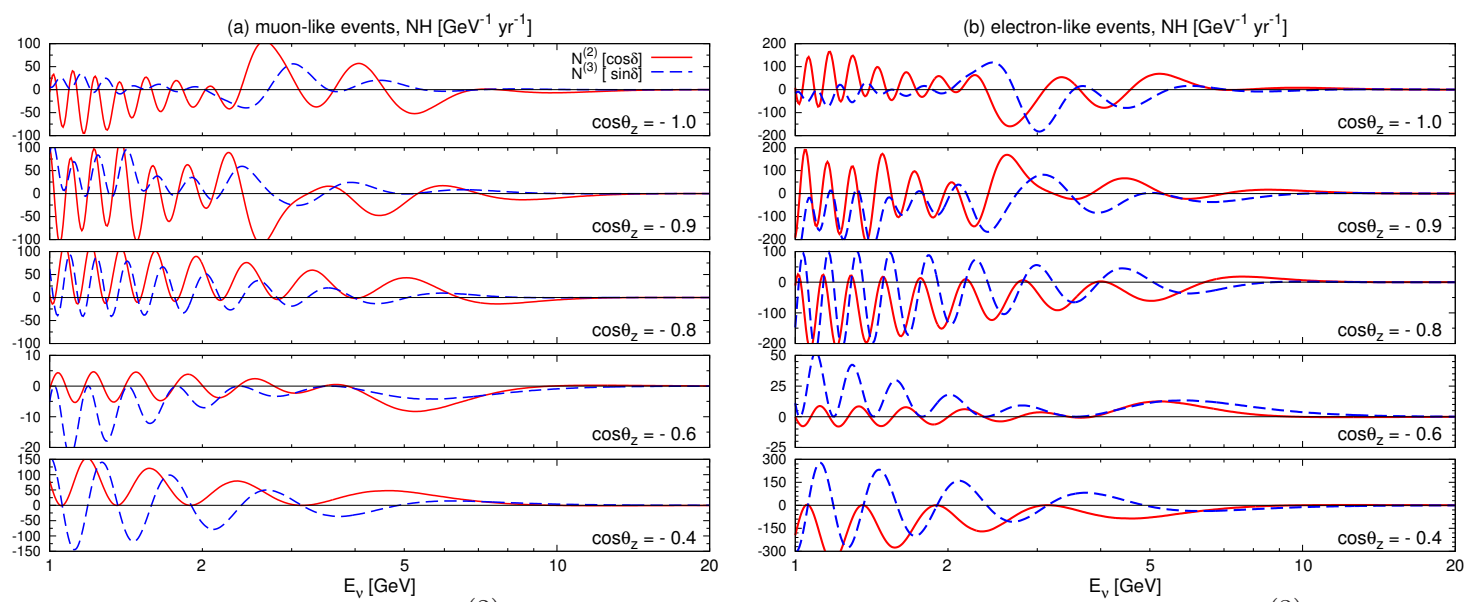

Figure 5. The coefficients $N_{\alpha}^{(2)}$ of $\cos \delta^{\prime}=\sqrt{1-x_{\mathrm{a}}^{2}} \cos \delta$ [red-solid lines] and $N_{\alpha}^{(3)}$ of $\sin \delta^{\prime}=$ $\sqrt{1-x_{\mathrm{a}}^{2}} \sin \delta$ [blue-dashed lines] for the muon-like $(\alpha=\mu)$ events (a) and electron-like events (b) for $\mathrm{NH}$.

called parametric resonance [61-63, 86-95]. Because of the large event numbers, there is a possibility that these differences can be identified in experiments and that the neutrino mass hierarchy is determined. We note, however, that the finite energy and angular resolution of real experiments may make it difficult to identify differences which depend strongly on the energy, such as those in the oscillation phase observed at $E_{\nu} \lesssim 4 \mathrm{GeV}$ at all $\cos \theta_{\mathrm{z}}$. On the other hand, the hierarchy dependence of the electron-like event rate $N_{\mathrm{e}}^{(0)}$, shown also by thick-red lines in figure 4(b), has little dependence on $\cos \theta_{\mathrm{z}}$ and does not oscillate in $E_{\nu}$. Although both the overall rate and the difference is small, the event is consistently higher for $\mathrm{NH}$ than $\mathrm{IH}$ in the broad energy range of $2 \sim 10 \mathrm{GeV}$, reflecting the MSW and parametric enhancements of the $\nu_{\mu} \rightarrow \nu_{\mathrm{e}}$ oscillation, $P_{\mu \mathrm{e}}^{(0)}=\frac{1}{2}\left(1-\left|S_{11}^{\prime}\right|^{2}\right)$, for NH; see $(2.32)$ and figure $1(\mathrm{a})$. Such moderate $E_{\nu}$ and $\cos \theta_{\mathrm{z}}$ dependences of the electron-like event rate on the mass hierarchy may allow actual experiments to identify the difference.

Let us now examine the coefficients $N_{\mu}^{(1)}$ and $N_{\mathrm{e}}^{(1)}$ of $x_{\mathrm{a}}$, which are shown by thin-blue lines in figure 4(a) and (b), respectively, also in solid for $\mathrm{NH}$ and in dashed for IH. Note that $N_{\mu}^{(1)}$ is positive definite while $N_{\mathrm{e}}^{(1)}$ tends to be negative at high energies $\left(E_{\nu} \gtrsim 2 \mathrm{GeV}\right)$. The coefficients $N_{\mu}^{(1)}$ and $N_{\mathrm{e}}^{(1)}$ are both proportional to $\frac{1}{2}\left(1-\left|S_{11}^{\prime}\right|^{2}\right)$ in the approximation of (2.32), and hence the energy-angular dependences are mild especially at high energy region of $4 \sim 10 \mathrm{GeV}$, just like the electron-like event rate $N_{\mathrm{e}}^{(0)}$. This will help experiments to measure $x_{\mathrm{a}}$. Because of the positive sign of $N_{\mu}^{(1)}$, the mass hierarchy determination by using only the muon-like events should be easier for $x_{\mathrm{a}}<0\left(\sin ^{2} \theta_{\mathrm{a}}<0.5\right)$ than for $x_{\mathrm{a}}>0\left(\sin ^{2} \theta_{\mathrm{a}}>0.5\right)$. The trend can be reversed when the electron-like events are also included because $N_{\mathrm{e}}^{(1)}$ has negative sign and has relatively larger magnitude. Likewise, $x_{\mathrm{a}}$ will be measured more accurately for $\mathrm{NH}$ than for $\mathrm{IH}$ when only the muon-like events are studied, whereas the measurement for IH can be significantly improved by including the electron-like events in the analysis.

The dependence on the CP phase $\delta$ is shown in figure 5 and figure 6 , respectively, for $\mathrm{NH}$ and IH. In both figures, the $N_{\alpha}^{(2)}$ [red-solid lines] coefficient of $\sqrt{1-x_{\mathrm{a}}^{2}} \cos \delta$ and $N_{\alpha}^{(3)}$ [blue-dashed lines] of $\sqrt{1-x_{\mathrm{a}}^{2}} \sin \delta$ are shown for the muon-like events in the left (a) and 

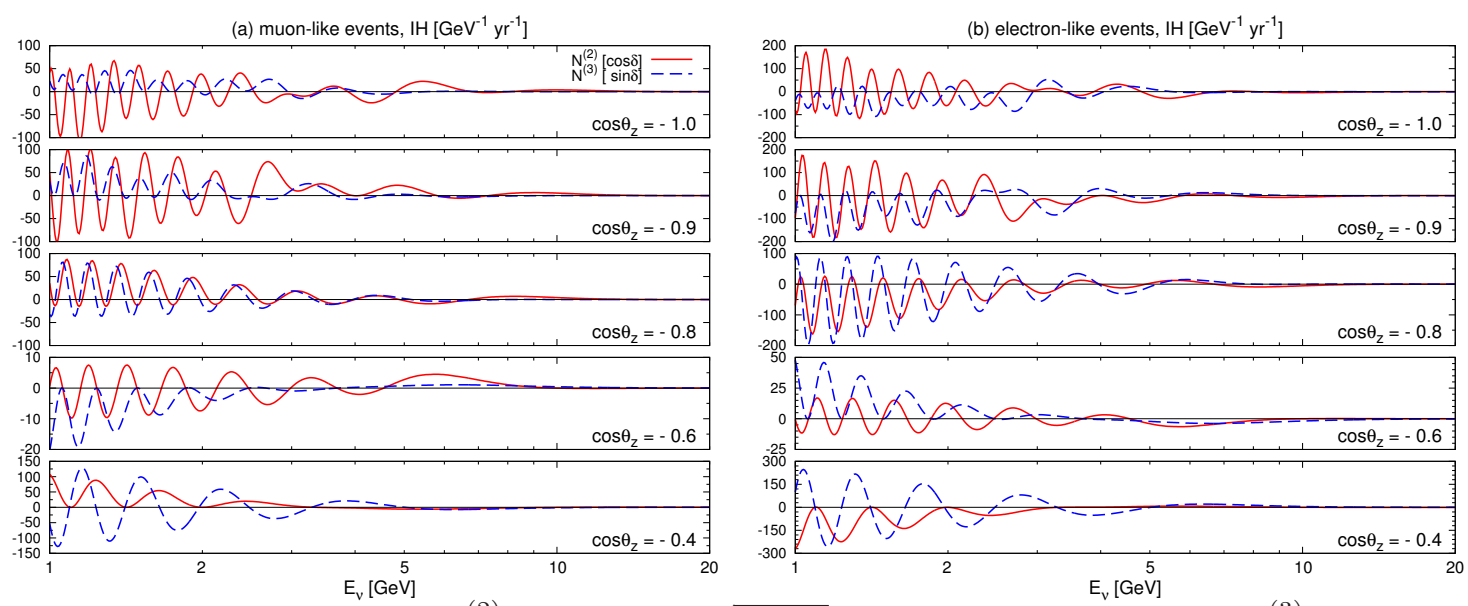

Figure 6. The coefficients $N_{\alpha}^{(2)}$ of $\cos \delta^{\prime}=\sqrt{1-x_{\mathrm{a}}^{2}} \cos \delta$ [solid-red lines] and $N_{\alpha}^{(3)}$ of $\sin \delta^{\prime}=$ $\sqrt{1-x_{\mathrm{a}}^{2}} \sin \delta$ [dashed-blue lines] for the muon-like $(\alpha=\mu)$ events (a) and electron-like events (b) for IH.

for the electron-like events in the right (b) panels. Let us first examine the $\delta$-dependence in the $\mathrm{NH}$ case as shown in figure 5 . We first note the significantly smaller magnitudes of the coefficients $N_{\alpha}^{(2)}$ and $N_{\alpha}^{(3)}$, which are typically $100 / \mathrm{GeV}$, as compared to $N_{\alpha}^{(0)}$ and $N_{\alpha}^{(1)}$ which are measured in unit of $1000 / \mathrm{GeV}$ as shown in figure 4 . If we restrict our attention to the higher energy region of $E_{\nu}>4 \mathrm{GeV}$ which is less sensitive to the experimental energyangular smearing effects, the electron-like events in figure 5(b) have higher sensitivity to both $\cos \delta$ [red-solid lines] and $\sin \delta$ [blue-dashed lines] than the muon-like events in figure 5(a). All the four coefficients $N_{\alpha}^{(2)}$ and $N_{\alpha}^{(3)}$ for $\alpha=\mu$ and $\alpha=e$ have larger magnitudes at lower energies, $E_{\nu} \lesssim 3 \mathrm{GeV}$, although they oscillate rapidly with $E_{\nu}$. The expected energy resolution of the PINGU detector may smear out those rapid oscillation. However, in a certain $\cos \theta_{\mathrm{z}}$ region the coefficients tend to have a definite sign which may survive after the energy smearing. For instance, let us examine the $\sin \delta^{\prime}$ measurement by using the coefficients $N_{\alpha}^{(3)}$ shown by blue-dashed lines in figure 5 . The average of $N_{\mu}^{(3)}$ at $\cos \theta_{\mathrm{z}}=-0.9$ is clearly positive in the whole energy range shown in the figures, whereas that of $N_{\mathrm{e}}^{(3)}$ tends to be negative in the whole region. They tend to oscillate about zero at $\cos \theta_{\mathrm{z}}=-1.0$, and the sign reverses at $\cos \theta_{\mathrm{z}}=-0.6$. Therefore, if the angular resolution of experiments can resolve $\cos \theta_{\mathrm{z}}=-0.9\left(\theta_{\mathrm{z}} \sim 154^{\circ}\right)$ from $\cos \theta_{\mathrm{z}}=-0.6\left(\theta_{\mathrm{z}} \sim 127^{\circ}\right)$, then it might be possible to measure $\sin \delta^{\prime}$ by using the total number of events including the low energy region. The same applies for the $\cos \delta^{\prime}$ measurements, for which the coefficient $N_{\mu}^{(2)}$ [red-solid lines] tends to be positive at around $\cos \theta_{\mathrm{z}}=-0.8$ and at -0.4 , while the opposite trend is expected for $N_{\mathrm{e}}^{(2)}$. Although a quantitative study with realistic event simulation is beyond the scope of the present paper, probability of using the low energy data for measuring $\delta$ may worth serious studies.

In case of IH, the coefficients $N_{\alpha}^{(2)}$ and $N_{\alpha}^{(3)}$ behave as in figure 6(a) and (b), respectively, for muon-like $(\alpha=\mu)$ and electron-like $(\alpha=e)$ events. Although the general trend of the oscillation patterns looks very similar between figure 5 for $\mathrm{NH}$ and figure 6 for $\mathrm{IH}$, the magnitude of the coefficients at high energies $\left(E_{\nu}>4 \mathrm{GeV}\right)$ are significantly smaller for IH than those for $\mathrm{NH}$. We should therefore expect that the $\delta$ measurement is more 

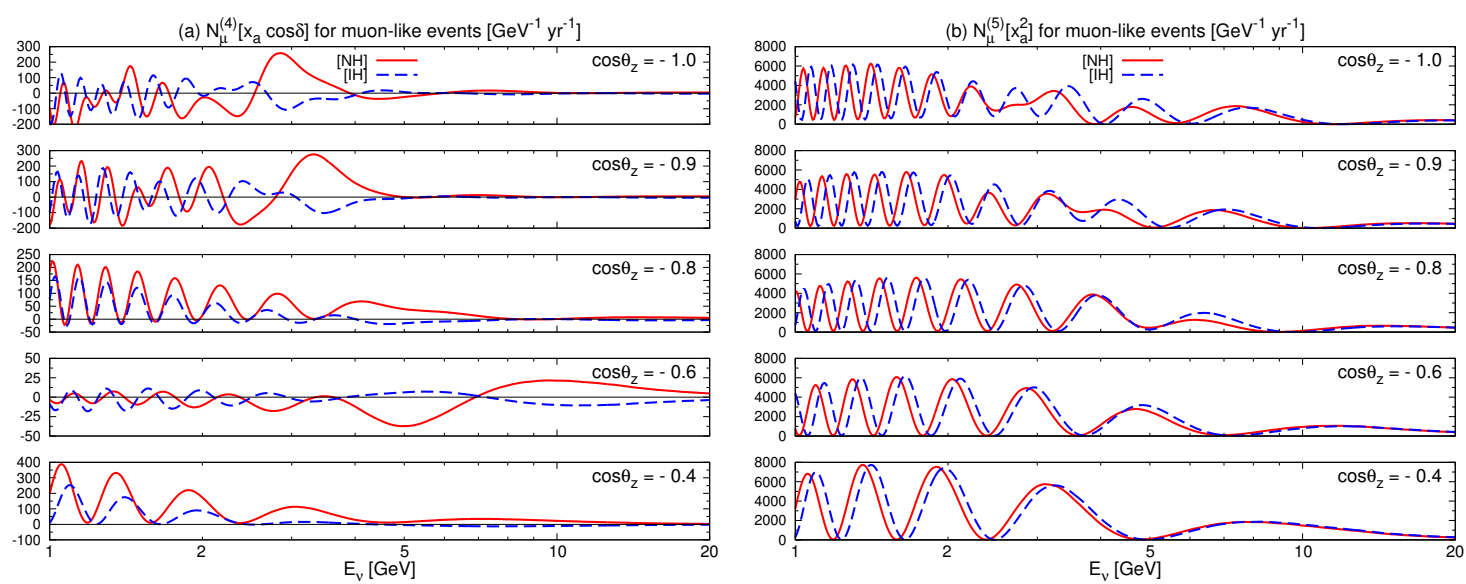

Figure 7. (a) The coefficients $N_{\mu}^{(4)}$ of $x_{\mathrm{a}} \cos \delta^{\prime}=x_{\mathrm{a}} \sqrt{1-x_{\mathrm{a}}^{2}} \cos ^{2} \delta$, and (b) $N_{\mu}^{(5)}$ of $x_{\mathrm{a}}^{2}$ for both $\mathrm{NH}$ [solid lines] and IH [dashed lines].

difficult for IH than for NH, when higher energy data are used. On the other hand, the $\cos \theta_{z}$ dependence of the sign and the magnitude of the coefficients integrated over the low energy region below $4 \mathrm{GeV}$ are similar to those of $\mathrm{NH}$ for $\cos \theta_{\mathrm{z}} \gtrsim-0.6$. Dedicated studies with realistic energy and angular resolution may reveal the possibility of measuring $\delta$ even in the IH case.

We show the coefficient $N_{\mu}^{(4)}$ of the cross term $x_{\mathrm{a}} \cos \delta^{\prime}=x_{\mathrm{a}} \sqrt{1-x_{\mathrm{a}}^{2}} \cos \delta$ and the coefficient $N_{\mu}^{(5)}$ of the quadratic term $x_{\mathrm{a}}^{2}$ in figure $7(\mathrm{a})$ and (b), respectively, for NH [redsolid lines] and IH [blue-dashed lines]. The magnitude of the coefficient $N_{\mu}^{(4)}$ is similar to those of $N_{\alpha}^{(2)}$ and $N_{\alpha}^{(3)}$ for $\cos \delta$ and $\sin \delta$ in figure 5 and figure 6 , as expected from an order of magnitude estimates in (2.30). Also, its energy dependence is similar to that of $\cos \delta$ coefficient $N_{\mu}^{(2)}$. We therefore expect that the uncertainty of the $\cos \delta$ measurement depends on the sign of $x_{\mathrm{a}}$ when the muon-like events are used in the analysis.

The magnitude of the coefficient $N_{\mu}^{(5)}$ of $x_{\mathrm{a}}^{2}$ in figure $7(\mathrm{~b})$ is of the same order of magnitude as that of $N_{\alpha}^{(1)}$ for the linear term in figure 4 . Note that the maximum value of the coefficient $N_{\mu}^{(1)}$ of $x_{\mathrm{a}}$ in figure $4(\mathrm{a})$ is around $140,250,690$, and 570 for $\cos \theta_{\mathrm{z}}=-1$, $-0.9,-0.8$, and -0.6 , respectively, for $\mathrm{NH}$, whereas those of the $\mathrm{IH}$ are smaller. On the other hand, the maximal values of the $x_{\mathrm{a}}^{2}$ coefficient $N_{\mu}^{(5)}$ in figure $7(\mathrm{~b})$ are 1800, 1870, 1264 , and 1050, for the same region. Around the peak of $N_{\mu}^{(5)}$, its magnitude is typically more than one order of magnitude larger than that of $N_{\mu}^{(1)}$, the term $N_{\mu}^{(5)} x_{\mathrm{a}}^{2}$ can be as sensitive to $x_{\mathrm{a}}$ as the term $N_{\mu}^{(1)} x_{\mathrm{a}}$ even when $x_{\mathrm{a}} \sim 0.2$.

\section{A simple $\chi^{2}$ analysis}

In this section, we examine the potential sensitivity of the PINGU experiment to the three unknown neutrino oscillation parameters at the neutrino event level. In other words, we assume that both the neutrino energy $\left(E_{\nu}\right)$ and its momentum direction $\left(\cos \theta_{\mathrm{z}}\right)$ are measured exactly for each event, and by ignoring uncertainties in the neutrino flux, cross sections and effective fiducial volume, the probability of misidentifying $\mu^{ \pm}$and $e^{ \pm}$events, as well as backgrounds from $\tau$-decay and neutral current events. Although these assumptions are 
far from reality, the results are still useful in identifying the maximum information hidden in the data, motivating and directing studies with full detector simulations. Especially, it can demonstrate that our decomposition method in the propagation basis is extremely powerful to reveal the hidden patterns behind the neutrino oscillogram.

\section{$4.1 \chi^{2}$ function}

We introduce a conventional $\chi^{2}$ technique to investigate experimental sensitivities on the neutrino mass hierarchy, the atmospheric mixing angle $\theta_{\mathrm{a}}$ and its octant, as well as the CP phase $\delta$. Note that the result of this method, $\chi_{\min }^{2}$ corresponds to the so-called "average experiment" [116] or "Asimov data set" [117]. The uncertainty from statistical fluctuation can be easily estimated to be $\Delta\left(\chi_{\min }^{2}\right) \approx 2 \sqrt{\Delta\left(\chi_{\min }^{2}\right)}[118-120]$. It not only applies to the case of discrete variables such as the neutrino mass hierarchy and the octant, but actually applies generally as long as the binned event number is large enough such that statistical fluctuation can be approximated by Gaussian distribution. Based on these two key parameters, $\chi_{\min }^{2}$ and its own variation $\Delta\left(\chi_{\min }^{2}\right)$, statistical interpretation can be made.

The $\chi^{2}$ function receives contributions from the statistical uncertainty of the event numbers as functions of the neutrino energy $E_{\nu}$ and its momentum direction $\cos \theta_{\mathrm{z}}$,

$$
\chi^{2} \equiv \sum_{\alpha} \int d E_{\nu} d \cos \theta_{\mathrm{z}}\left[\frac{\left(\frac{d N_{\alpha}}{d E_{\nu} d \cos \theta_{\mathrm{z}}}\right)^{\mathrm{th}}-\left(\frac{d N_{\alpha}}{d E_{\nu} d \cos \theta_{\mathrm{z}}}\right)^{\mathrm{obs}}}{\sqrt{\left(\frac{d N_{\alpha}}{d E_{\nu} d \cos \theta_{\mathrm{z}}}\right)^{\mathrm{obs}}}}\right]^{2}+\chi_{\text {para }}^{2}
$$

as well as external constraint on neutrino oscillation parameters which has been denoted as $\chi_{\text {para }}^{2}$,

$$
\begin{aligned}
\chi_{\text {para }}^{2}= & {\left[\frac{\left(\delta m_{\mathrm{a}}^{2}\right)^{\mathrm{fit}}-\overline{\delta m_{\mathrm{a}}^{2}}}{\Delta \delta m_{\mathrm{a}}^{2}}\right]^{2}+\left[\frac{\left(\delta m_{\mathrm{s}}^{2}\right)^{\mathrm{fit}}-\overline{\delta m_{\mathrm{s}}^{2}}}{\Delta \delta m_{\mathrm{s}}^{2}}\right]^{2} } \\
& +\left[\frac{\left(\sin ^{2} 2 \theta_{\mathrm{r}}\right)^{\mathrm{fit}}-\overline{\sin ^{2} 2 \theta_{\mathrm{r}}}}{\Delta \sin ^{2} 2 \theta_{\mathrm{r}}}\right]^{2}+\left[\frac{\left(\sin ^{2} 2 \theta_{\mathrm{s}}\right)^{\mathrm{fit}}-\overline{\sin ^{2} 2 \theta_{\mathrm{s}}}}{\Delta \sin ^{2} 2 \theta_{\mathrm{s}}}\right]^{2}+\left[\frac{\left(\sin ^{2} 2 \theta_{\mathrm{a}}\right)^{\mathrm{fit}}-\overline{\sin ^{2} 2 \theta_{\mathrm{a}}}}{\Delta \sin ^{2} 2 \theta_{\mathrm{a}}}\right]^{2},
\end{aligned}
$$

with the current central values and expected uncertainties in the near future [109, 111-115],

$$
\begin{array}{ll}
\delta m_{\mathrm{a}}^{2}=2.35 \pm 0.1 \times 10^{-3} \mathrm{eV}^{2}, & \sin ^{2} 2 \theta_{\mathrm{s}}=0.857 \pm 0.024 \\
\delta m_{\mathrm{s}}^{2}=7.50 \pm 0.2 \times 10^{-5} \mathrm{eV}^{2}, & \sin ^{2} 2 \theta_{\mathrm{r}}=0.098 \pm 0.005 \\
& \sin ^{2} 2 \theta_{\mathrm{a}}=0.957 \pm 0.030
\end{array}
$$

We generate events with the mean values of the parameters in (4.3) for one of the mass hierarchies, except for $x_{\mathrm{a}}=\cos ^{2} \theta_{\mathrm{a}}-\sin ^{2} \theta_{\mathrm{a}}$, for which we examine three input values \pm 0.2 and 0 , which are consistent with the present constraints. As for the CP phase $\delta$, we examine four cases, $0, \pi$, and $\pm \pi / 2$. We then use MINUIT [121] to find the minimum of the $\chi^{2}$ function by varying all the six parameters, $\delta m_{\mathrm{a}}^{2}, \delta m_{\mathrm{s}}^{2}, \sin ^{2} 2 \theta_{\mathrm{s}}, \sin ^{2} 2 \theta_{\mathrm{r}}, x_{\mathrm{a}}$, and $\delta$. The dependence on the true values of the neutrino oscillation parameters is consistent with those in the previous studies [38, 39, 45, 46, 69, 70]. 


\begin{tabular}{|c|l|ccc|ccc|}
\hline \multicolumn{2}{|c|}{$\Delta \chi_{\mathrm{MH}}^{2}$} & \multicolumn{3}{c|}{$\mathrm{NH}$} & \multicolumn{3}{c|}{$\mathrm{IH}$} \\
\hline \multirow{4}{*}{$\mu$-like } & $\bar{x}_{\mathrm{a}}=-0.2$ & $\bar{x}_{\mathrm{a}}=0.0$ & $\bar{x}_{\mathrm{a}}=+0.2$ & $\bar{x}_{\mathrm{a}}=-0.2$ & $\bar{x}_{\mathrm{a}}=0.0$ & $\bar{x}_{\mathrm{a}}=+0.2$ \\
& $\bar{\delta}=0^{\circ}$ & 163.0 & 174.9 & 141.8 & 100.7 & 109.7 & 96.7 \\
& $\bar{\delta}=90^{\circ}$ & 170.3 & 183.3 & 151.5 & 99.9 & 110.2 & 97.4 \\
& $\bar{\delta}=180^{\circ}$ & 168.5 & 179.7 & 152.1 & 98.0 & 108.1 & 96.5 \\
& $\bar{\delta}=270^{\circ}$ & 160.0 & 171.0 & $\mathbf{1 4 1 . 5}$ & 98.6 & 107.8 & $\mathbf{9 5 . 6}$ \\
\hline \multirow{4}{*}{$\mu+$ e-like } & $\bar{\delta}=0^{\circ}$ & 252.9 & 215.3 & 168.9 & 143.5 & 140.7 & 120.1 \\
& $\bar{\delta}=90^{\circ}$ & 255.9 & 219.2 & 172.0 & 141.0 & 140.2 & 119.7 \\
& $\bar{\delta}=180^{\circ}$ & 256.6 & 218.2 & 171.5 & 136.4 & 135.9 & $\mathbf{1 1 5 . 6}$ \\
& $\bar{\delta}=270^{\circ}$ & 252.8 & 213.4 & $\mathbf{1 6 6 . 8}$ & 139.0 & 136.9 & 116.8 \\
\hline
\end{tabular}

Table 1. The dependence of hierarchy sensitivity $\Delta \chi_{\mathrm{MH}}^{2}$ on the input values of the atmospheric angle's deviation from $45^{\circ}, \bar{x}_{\mathrm{a}}$, and the CP phase, $\bar{\delta}$, with 1-year running of PINGU. The cases of both $\mathrm{NH}$ and $\mathrm{IH}$, muon- and electron-like events have been considered with event cut $E_{\nu}>4 \mathrm{GeV}$ and $\cos \theta_{\mathrm{z}}<-0.4$.

Not all the information in atmospheric neutrino mixing pattern can be retrieved after reconstructing the events. The mixing pattern with low energy and small $\left|\cos \theta_{z}\right|$ will be lost due to smearing and detector resolution. To see how this would affect the result, we will apply simple event cuts on neutrino energy $E_{\nu}$ and the zenith angle $\theta_{z}$ when presenting the results below.

\subsection{The mass hierarchy}

As shown in figure 1, the neutrino mass hierarchy can be determined by observing the MSW resonances due to the Earth matter effect which occurs only for NH in neutrino oscillations and for IH in antineutrino oscillations. Although the differences are partially cancelled for a detector like PINGU which is incapable of distinguishing neutrino from antineutrino, it is still possible to determine the neutrino mass hierarchy with atmospheric neutrino oscillation because of incomplete cancellation. The hierarchy can be defined as,

$$
\Delta \chi_{\mathrm{MH}}^{2} \equiv\left|\chi_{\min }^{2}(\mathrm{NH})-\chi_{\min }^{2}(\mathrm{IH})\right|,
$$

where the $\chi^{2}$ minimum, $\chi_{\min }^{2}(\mathrm{NH})$ or $\chi_{\min }^{2}(\mathrm{IH})$, is obtained by setting the neutrino mass hierarchy to be normal $(\mathrm{NH})$ or inverted $(\mathrm{IH})$.

Since the CP phase $\delta$ and the atmospheric mixing angle $\theta_{\mathrm{a}}$ have not been pinned down yet, their values would affect the distinguishability of the mass hierarchy. The dependence of the hierarchy sensitivity $\Delta \chi_{\mathrm{MH}}^{2}$ on the input values of $\delta$ and the parameter $x_{\mathrm{a}}=\cos ^{2} \theta_{\mathrm{a}}-$ $\sin ^{2} \theta_{\mathrm{a}}$ is summarized in table 1 . Four typical cases of $\bar{\delta}=\frac{n}{2} \pi$ with $n=0,1,2,3$ respectively and three possibilities for $\bar{x}_{\mathrm{a}}= \pm 0.2,0.0$ have been shown for both $\mathrm{NH}$ in the left and IH in the right. Since muon-like events are easier to be measured, we first show the results with only muon-like events in the upper part and then also include the electron-like events in the lower part.

The results in table 1 are obtained with the event cuts $E_{\nu}>4 \mathrm{GeV}$ and $\cos \theta_{\mathrm{z}}<-0.4$. Even with this limited parameter space, the hierarchy sensitivity $\Delta \chi_{\mathrm{MH}}^{2}$ is sizable, being 
larger than 141 for $\mathrm{NH}$ and 98 for $\mathrm{IH}$ for all cases of input values for $\delta$ and $\theta_{\mathrm{a}}$, under the assumption of a perfect detector. The smallest value of $\Delta \chi_{\mathrm{MH}}^{2}$ in each block has been marked as bold numbers. For most cases, the hierarchy sensitivity is larger for NH than IH. This is because the muon-like event rate with $\mathrm{NH}$ is smaller than the one with IH in the considered energy range, as shown in figure 4 . The largest contribution to the muon-like event rate comes from the $\nu_{\mu}$ flux $\phi_{\nu_{\mu}}$ as shown in figure 8. For NH, neutrinos experience resonances, significantly reducing the $\mu$-like event rate. This trend remains, even after including the electron-like event rate which is larger for $\mathrm{NH}$.

The dependence on $x_{\mathrm{a}}$ is a little more complicated due to the presence of both the linear and quadratic terms. Let us first compare the results at $\bar{x}_{\mathrm{a}}=+0.2$ and $\bar{x}_{\mathrm{a}}=-0.2$ which have the same contribution from the quadratic term. So the difference between them is caused by the linear term. For NH, the hierarchy sensitivity is larger for negative $\bar{x}_{\mathrm{a}}$. This is because in most part of the energy and zenith angle range under consideration, especially in the regions $\cos \theta_{\mathrm{z}} \gtrsim-0.7$ and $\cos \theta_{\mathrm{z}} \lesssim-0.9$, negative $x_{\mathrm{a}}$ makes the difference between $\mathrm{NH}$ and IH larger. In other words, the sensitivity increases with $\sin ^{2} \theta_{23}$ and decreases with $\bar{x}_{\mathrm{a}}$ for both muon- and electron-like events. For IH, the linear term coefficients are much smaller, as shown in figure 4 . Consequently, the difference between $\bar{x}_{\mathrm{a}}=+0.2$ and $\bar{x}_{\mathrm{a}}=-0.2$ is small. If only linear term of $\bar{x}_{\mathrm{a}}$ is present, the dependence should be monotonically decreasing with $\bar{x}_{\mathrm{a}}$, rendering the hierarchy sensitivity at $\bar{x}_{\mathrm{a}}=0$ to be between the values at $\bar{x}_{\mathrm{a}}=-0.2$ and $\bar{x}_{\mathrm{a}}=-0.2$. This monotonic trend receives correction from the quadratic term $\bar{x}_{\mathrm{a}}^{2}$. The coefficient $N_{\mu}^{(5)}$ of the quadratic term is always positive and its hierarchy dependence is the opposite to that of $N_{\mu}^{(0)}$, leading to a negative contribution to the hierarchy sensitivity. As we mentioned earlier, the main contribution comes from the minimum in the energy range of $6 \mathrm{GeV} \lesssim E_{\nu} \lesssim 10 \mathrm{GeV}$ where the quadratic term can dominate. So the hierarchy sensitivity at $\bar{x}_{\mathrm{a}}= \pm 0.2$ is suppressed by the quadratic term. In other words, the sensitivity at $\bar{x}_{\mathrm{a}}=0$ is effectively lifted. This contribution from the quadratic term can be strong enough to make the hierarchy sensitivity to be the largest at $\bar{x}_{\mathrm{a}}=0$. Note that this only happens for the case with only muon-like events since there is no quadratic term for electron-like events. When the later is also included, the dependence on $x_{\mathrm{a}}$ becomes monotonically decreasing with $x_{\mathrm{a}}$, in other words, increasing with $\theta_{\mathrm{a}}$.

The dependence on the CP phase $\delta$ is much smaller, because the corresponding coefficients $N_{\alpha}^{(2)}$ of $\cos \delta^{\prime}$ and $N_{\alpha}^{(3)}$ of $\sin \delta^{\prime}$ are only around $3 \%$ of $N_{\alpha}^{(0)}, N_{\alpha}^{(1)}$, and $N_{\mu}^{(5)}$, as shown in figure 4 , figure 5 , figure 6 , and figure 7 . For muon-like events with $\mathrm{NH}$, the contribution from $N_{\mu}^{(2)}$ is the opposite to and the one from $N_{\mu}^{(3)}$ is the same as $N_{\mu}^{(0)}$, as shown in figure 4 and figure 5, rendering smaller hierarchy sensitivity for positive $\cos \delta$ and negative $\sin \delta$ as recorded in table 1 . Note that this trend is reversed for IH and the dependence is much smaller.

In the upper-left block of table 1 for $\mathrm{NH}$ with only muon-like events, the dependence on $\delta$ is smaller than that on $x_{\mathrm{a}}$. For each row with fixed input value of $\delta$, the variation is around $16 \sim 21$, while for each column with fixed input value of $x_{\mathrm{a}}$, it is around $10 \sim 12$. This property applies for all the other three blocks. In the upper-right block for IH with only muon-like events, the variant in rows is around $12 \sim 13$, but the variant in columns is much smaller, being around $2 \sim 3$. Such trends are expected since the magnitude of the 
observable coefficients of $x_{\mathrm{a}}$, namely $N_{\mu}^{(1)}$, is much larger than those of $\cos \delta^{\prime}$ and $\sin \delta^{\prime}$, $N_{\mu}^{(2)}$ and $N_{\mu}^{(3)}$, respectively. The variation in $x_{\text {a }}$ further increases after electron-like events are included since the ratio of coefficients $N_{\mathrm{e}}^{(1)} / N_{\mathrm{e}}^{(0)}$ is more significant than $N_{\mu}^{(1)} / N_{\mu}^{(0)}$ as shown in figure 4 . The $x_{\mathrm{a}}$-dependence of $\chi_{\mathrm{MH}}^{2}$ is consistently larger for $\mathrm{NH}$ than for $\mathrm{IH}$, because the coefficient $N_{\alpha}^{(1)}$ of $x_{\mathrm{a}}$ is larger for $\mathrm{NH}$ than for IH, as shown in figure 4. It is remarkable that when electron-like events are included in the analysis, the hierarchy distinguishing power increases significantly for $x_{\mathrm{a}}=-0.2$, but not much for $x_{\mathrm{a}}=+0.2$. This is because of the negative sign of $N_{\mathrm{e}}^{(1)}$, shown in figure 4(b), which enlarges the hierarchy dependence of the event rate for negative $x_{\mathrm{a}}$.

Although the absolute magnitude of $\Delta \chi_{\mathrm{MH}}^{2}$ in table 1 for a perfect detector without systematic uncertainty do not have much significance, the relative importance of electronlike events and possible impacts of the $x_{\mathrm{a}}$ value in the hierarchy determination may want further studies. Note that the neutrino mass hierarchy can be resolved no matter what true values of the atmospheric angle and the $\mathrm{CP}$ phase can be, in contrast to the $\mathrm{CP}$-hierarchy and octant-hierarchy degeneracies from which accelerator based neutrino experiments suffer $[122-124]$.

Those events at low energy and/or small $\left|\cos \theta_{z}\right|$ will be largely smeared out, and oscillating features may be averaged out. This is because the energy smearing mainly comes from the inexact energy reconstruction procedure, which is expected to scale as a linear function $\delta E \propto E$, and statistical fluctuation, which scales as $\delta E \propto \sqrt{E}$. On the other hand, the neutrino oscillation period shrinks quickly at low energy, approximately as a quadratic function $\Delta E \propto E^{2}$. In other words, the energy resolution becomes larger than the oscillation period when the neutrino energy is low enough. No oscillation signal can be expected to survive below some energy threshold. Given neutrino energy, the angular resolution, $\delta \theta_{\mathrm{z}}$, is constant in the neutrino frame, no matter where the neutrino comes from. When converted to the earth frame, the resolution, $\delta\left(\cos \theta_{\mathrm{z}}\right)=\sin \theta_{\mathrm{z}} \delta \theta_{\mathrm{z}}$, is much larger for horizontal events, $\sin \theta_{\mathrm{z}} \approx 1$. Hence, these regions may not contribute after smearing which can be approximated by simply applying event selection cuts $E_{\nu}>E_{\nu}^{\text {cut }}$ and $\cos \theta_{\mathrm{z}}<\cos \theta_{\mathrm{z}}^{\text {cut }}$.

The dependences on $E_{\nu}^{\text {cut }}$ and $\cos \theta_{\mathrm{z}}^{\text {cut }}$ are shown in table 2 where the input values of $\delta$ and $x_{\mathrm{a}}$ are chosen corresponding to the smallest value of $\Delta \chi_{\mathrm{MH}}^{2}$ in each block of table 1 respectively. We observe that the results depend strongly on the range of $E_{\nu}$ and $\cos \theta_{z}$. For instance, when $E_{\nu}^{\text {cut }}$ is raised from $6 \mathrm{GeV}$ to $8 \mathrm{GeV}, \Delta \chi_{\mathrm{MH}}^{2}$ drops by nearly a factor of $3 \sim 7$, while for $E_{\nu}>8 \mathrm{GeV}$, changing of the zenith angle coverage from $\cos \theta_{\mathrm{z}}<-0.4$ to $\cos \theta_{\mathrm{z}}<-0.6$ reduces $\Delta \chi_{\mathrm{MH}}^{2}$ by further factor of 2 . It is therefore very important to have low energy threshold of the detector and to study the smearing effects in detail.

\subsection{The atmospheric angle and its octant}

Once the mass hierarchy is determined, $\chi^{2}$ fit can be performed with the correct mass hierarchy as an input, and the same $\chi^{2}$ function (4.1) can be used to measure the atmospheric mixing angle and its octant discussed here, as well as the CP phase $\delta$ which will be discussed in section 4.4. In order to make the global minimum to be at the input value 


\begin{tabular}{|c|l|ccc|ccc|}
\hline \multicolumn{2}{|c|}{$\Delta \chi_{\mathrm{MH}}^{2}$} & \multicolumn{3}{|c|}{$\mathrm{NH}$} & \multicolumn{3}{c|}{$\mathrm{IH}$} \\
\hline \multirow{5}{*}{$\mu$-like } & $\cos \theta_{\mathrm{z}}<-0.2$ & $\cos \theta_{\mathrm{z}}<-0.4$ & $\cos \theta_{\mathrm{z}}<-0.6$ & $\cos \theta_{\mathrm{z}}<-0.2$ & $\cos \theta_{\mathrm{z}}<-0.4$ & $\cos \theta_{\mathrm{z}}<-0.6$ \\
& $E_{\nu}>2 \mathrm{GeV}$ & 260.0 & 249.2 & 234.6 & 131.2 & 129.7 & 123.6 \\
& $E_{\nu}>4 \mathrm{GeV}$ & 143.2 & $\mathbf{1 4 1 . 5}$ & 125.0 & 98.9 & $\mathbf{9 5 . 6}$ & 91.4 \\
& $E_{\nu}>6 \mathrm{GeV}$ & 39.5 & 36.4 & 29.9 & 50.0 & 48.3 & 41.0 \\
& $E_{\nu}>8 \mathrm{GeV}$ & 9.9 & 9.0 & 4.8 & 15.6 & 13.4 & 6.3 \\
\hline \multirow{5}{*}{$\mu+\mathrm{e}-$-like } & $E_{\nu}>2 \mathrm{GeV}$ & 281.4 & 271.8 & 254.6 & 142.7 & 139.3 & 136.2 \\
& $E_{\nu}>4 \mathrm{GeV}$ & 169.5 & $\mathbf{1 6 6 . 8}$ & 148.3 & 117.6 & $\mathbf{1 1 5 . 6}$ & 112.7 \\
& $E_{\nu}>6 \mathrm{GeV}$ & 51.5 & 45.6 & 36.3 & 61.2 & 58.6 & 53.2 \\
& $E_{\nu}>8 \mathrm{GeV}$ & 12.0 & 10.6 & 5.7 & 21.0 & 19.1 & 13.6 \\
\hline
\end{tabular}

Table 2. The dependence of the hierarchy sensitivity $\Delta \chi_{\mathrm{MH}}^{2}$ on event selection cuts for 1-year running of PINGU. The cases of NH and IH, muon- and electron-like events, respectively, have been considered. The input values of $x_{\mathrm{a}}$ and $\delta$ are taken as those in table 1 that produces the smallest value of $\Delta \chi_{\mathrm{MH}}^{2}$ within the corresponding block, which have been labeled with bold numbers.

of $x_{\mathrm{a}}=\bar{x}_{\mathrm{a}}=x_{\mathrm{a}}^{\text {input }}$, we modify the last term in $\chi_{\text {para }}^{2}(4.2)$ as follows,

$$
\left[\frac{\left(\sin ^{2} 2 \theta_{\mathrm{a}}\right)^{\mathrm{fit}}-\overline{\sin ^{2} 2 \theta_{\mathrm{a}}}}{\Delta \sin ^{2} 2 \theta_{\mathrm{a}}}\right]^{2} \rightarrow\left[\frac{x_{\mathrm{a}}^{2}-\bar{x}_{\mathrm{a}}^{2}}{0.03}\right]^{2},
$$

which keeps the uncertainty in $\sin ^{2} 2 \theta_{\text {a }}$ the same as in (4.3) while shifting the mean value to the input, $\sin ^{2} 2 \theta_{\mathrm{a}}=1-\bar{x}_{\mathrm{a}}^{2}$. This avoids small but inessential dependence of the $x_{\mathrm{a}}$ measurement on the input values of $x_{\mathrm{a}}$ in the region $\left|x_{\mathrm{a}}\right|<0.2$.

\subsubsection{The atmospheric angle}

We compute the minimum of the $\chi^{2}$ function as a function of the parameter $x_{\mathrm{a}}$, by varying all the other 5 parameters with MINUIT2 [121], to obtain the region, $\chi_{\min }^{2}\left(x_{\mathrm{a}}\right) \leq 1$, whose half-width is defined as the expected uncertainty of the $x_{\mathrm{a}}$ measurement, $\Delta\left(x_{\mathrm{a}}\right)$ or $\Delta\left(x_{\mathrm{a}}^{2}\right)$. The results for $\mathrm{NH}$ and $\mathrm{IH}$ are listed in the left and right panels of table 3 , respectively. For each hierarchy, four input values $\bar{\delta}=0^{\circ}, 90^{\circ}, 180^{\circ}, 270^{\circ}$ of the CP phase $\delta$ and three input values $\bar{x}_{\mathrm{a}}=0.0, \pm 0.2$ for the atmospheric angle $\theta_{\mathrm{a}}$ have been tested. Both the uncertainty of $x_{\mathrm{a}}, \Delta\left(x_{\mathrm{a}}\right)$, and that of $x_{\mathrm{a}}^{2}, \Delta\left(x_{\mathrm{a}}^{2}\right)$, are given in table 3 .

Note that the uncertainty $\Delta\left(x_{\mathrm{a}}\right)$ is larger if the atmospheric mixing angle is maximal, or when $\bar{x}_{\mathrm{a}}=0\left(\sin ^{2} 2 \theta_{\mathrm{a}}=1\right)$. This is a consequence of relatively large coefficient $N_{\mu}^{(5)}$ of $x_{\mathrm{a}}^{2}$, shown in figure $7(\mathrm{~b})$, as compared to the coefficients $N_{\mu}^{(1)}$ and $N_{\mathrm{e}}^{(1)}$ of $x_{\mathrm{a}}$, shown in figure $4(\mathrm{a})$ and (b), respectively. The $x_{\mathrm{a}}$-dependence of the muon-like events are expected to be,

$$
x_{\mathrm{a}} N_{\mu}^{(1)}+x_{\mathrm{a}}^{2} N_{\mu}^{(5)}+\cdots,
$$

ignoring the other small terms. The variation of the number of events at $x_{\mathrm{a}}=\bar{x}_{\mathrm{a}}$ is then,

$$
\left(N_{\mu}^{(1)}+2 \bar{x}_{\mathrm{a}} N_{\mu}^{(5)}\right) \delta x_{\mathrm{a}}+\cdots .
$$

Since the $N_{\mu}^{(5)}$ term can dominate over $N_{\mu}^{(1)}$ and $N_{\mathrm{e}}^{(1)}$ for $\left|\bar{x}_{\mathrm{a}}\right|=0.2$, and it vanishes for $\bar{x}_{\mathrm{a}}=0$, the combined effective coefficient of $\delta x_{\mathrm{a}}$ has larger magnitude for nonzero $\bar{x}_{\mathrm{a}}$. This 


\begin{tabular}{|c|l|ccc|ccc|}
\hline \multicolumn{2}{|c|}{$\mu+\mathrm{e}$-like } & \multicolumn{3}{c|}{$\mathrm{NH}$} & \multicolumn{3}{c|}{$\mathrm{IH}$} \\
\hline \multirow{4}{*}{$\Delta\left(x_{\mathrm{a}}\right)$} & $\bar{x}_{\mathrm{a}}=-0.2$ & $\bar{x}_{\mathrm{a}}=0.0$ & $\bar{x}_{\mathrm{a}}=+0.2$ & $\bar{x}_{\mathrm{a}}=-0.2$ & $\bar{x}_{\mathrm{a}}=0.0$ & $\bar{x}_{\mathrm{a}}=+0.2$ \\
\hline & $\bar{\delta}=0^{\circ}$ & 0.012 & 0.025 & 0.010 & 0.012 & 0.035 & 0.011 \\
& $\bar{\delta}=90^{\circ}$ & 0.013 & 0.025 & 0.011 & 0.013 & 0.035 & 0.012 \\
& $\bar{\delta}=180^{\circ}$ & 0.012 & 0.026 & 0.010 & 0.012 & 0.035 & 0.011 \\
& $\bar{\delta}=270^{\circ}$ & 0.013 & 0.025 & 0.011 & 0.013 & 0.035 & 0.012 \\
\hline \multirow{4}{*}{$\Delta\left(x_{\mathrm{a}}^{2}\right)$} & $\bar{\delta}=0^{\circ}$ & 0.0049 & 0.00061 & 0.0041 & 0.0048 & 0.0012 & 0.0044 \\
& $\bar{\delta}=90^{\circ}$ & 0.0052 & 0.00065 & 0.0044 & 0.0052 & 0.0012 & 0.0046 \\
& $\bar{\delta}=180^{\circ}$ & 0.0048 & 0.00066 & 0.0042 & 0.0048 & 0.0013 & 0.0043 \\
& $\bar{\delta}=270^{\circ}$ & 0.0052 & 0.00065 & 0.0044 & 0.0052 & 0.0012 & 0.0046 \\
\hline
\end{tabular}

Table 3. The dependence of the uncertainty of $x_{\mathrm{a}}$ and $x_{\mathrm{a}}^{2}$, corresponding to $\chi_{\min }^{2}\left(x_{\mathrm{a}}\right) \leq 1$ and $\chi_{\min }^{2}\left(x_{\mathrm{a}}^{2}\right) \leq 1$, respectively, on the input values $\bar{x}_{\mathrm{a}}$ and $\bar{\delta}$ after 1 -year running of PINGU. The cases of both muon- and electron-like events for both $\mathrm{NH}$ and IH have been considered with event cuts $E_{\nu}>4 \mathrm{GeV}$ and $\cos \theta_{\mathrm{z}}<-0.4$.

explains the reduced uncertainty $\Delta\left(x_{\mathrm{a}}\right)$ for $x_{\mathrm{a}}= \pm 0.2$. The slight difference between the two mirror cases $\bar{x}_{\mathrm{a}}=-0.2$ and $\bar{x}_{\mathrm{a}}=0.2$ comes from the first term. Since $N_{\mu}^{(1)}$ and $N_{\mu}^{(5)}$ are positive, cancellation happens in the combined effective coefficient when $\bar{x}_{\mathrm{a}}$ is negative, leading to systematically larger $\Delta\left(x_{\mathrm{a}}\right)$. There is some slight dependence on the input value $\bar{\delta}$ of the CP phase, but not sizable.

To make a direct comparison with the external constraint (4.5), the resolution $\Delta\left(x_{\mathrm{a}}^{2}\right)$ is also shown in table 3 . Since $\sin ^{2} 2 \theta_{\mathrm{a}} \equiv 1-x_{\mathrm{a}}^{2}$, the uncertainty $\Delta\left(\sin ^{2} 2 \theta_{\mathrm{a}}\right)$ is exactly $\Delta\left(x_{\mathrm{a}}^{2}\right)$. For $x_{\mathrm{a}} \approx|0.2|$, it can be roughly estimated as $\Delta\left(x_{\mathrm{a}}^{2}\right) \approx\left(2 x_{\mathrm{a}}\right) \Delta\left(x_{\mathrm{a}}\right) \approx 0.4 \Delta\left(x_{\mathrm{a}}\right)$ which is typically a factor of $5 \sim 6$ smaller than the uncertainty of 0.03 given in (4.3) and (4.5). If the true value of $x_{\mathrm{a}}$ vanishes, its uncertainty should be estimated as $\Delta\left(x_{\mathrm{a}}^{2}\right) \approx\left[\Delta\left(x_{\mathrm{a}}\right)\right]^{2}$ which is roughly 0.0006 for $\mathrm{NH}$, which is smaller than the current uncertainty by a factor of 50. The uncertainties $\Delta\left(x_{\mathrm{a}}\right)$ and $\Delta\left(x_{\mathrm{a}}^{2}\right)$ are slightly larger for IH due to the smaller coefficients $N_{\alpha}^{(1)}$ as shown in figure 4 .

Summing up, a neutrino telescope like PINGU has a potential to reduce the uncertainty of $\sin ^{2} 2 \theta_{\mathrm{a}}$ by a factor of 5 to 50 within one year of running. Although our simulation does not take account of energy and zenith angle resolutions, we expect this high potential to be confirmed in more realistic simulations because the coefficients $N_{\mu}^{(1)}$ and $N_{\mathrm{e}}^{(1)}$ do not oscillate much with $E_{\nu}$ or $\cos \theta_{\mathrm{z}}$, consequently, can survive the smearing effects.

\subsubsection{Octant sensitivity}

If the linear terms $N_{\alpha}^{(1)}$ of $x_{\mathrm{a}}$ vanish, no difference would be observed when $x_{\mathrm{a}}$ switches its sign. Fortunately, the nonzero $N_{\mu}^{(1)}$ and $N_{\mathrm{e}}^{(1)}$ provide us the possibility of determining the octant of the atmospheric mixing angle. The octant sensitivity can be defined as,

$$
\Delta \chi_{\text {octant }}^{2} \equiv\left|\chi_{\min }^{2}\left(x_{\mathrm{a}}>0\right)-\chi_{\min }^{2}\left(x_{\mathrm{a}}<0\right)\right|,
$$

where the two $\chi_{\min }^{2}$ are obtained by restricting the atmospheric mixing angle in the higher or lower octant, respectively. The results with different input values, $\bar{x}_{\mathrm{a}}= \pm 0.2, \pm 0.1$ and $\bar{\delta}=0^{\circ}, 90^{\circ}, 180^{\circ}, 270^{\circ}$, have been shown in table 4 for both $\mathrm{NH}$ and $\mathrm{IH}$. 


\begin{tabular}{|c|c|c|c|c|c|c|c|c|c|}
\hline \multirow{2}{*}{\multicolumn{2}{|c|}{$\frac{\Delta \chi_{\text {octant }}^{2}}{\bar{x}_{\mathrm{a}}}$}} & \multicolumn{4}{|c|}{$\mathrm{NH}$} & \multicolumn{4}{|c|}{$\mathrm{IH}$} \\
\hline & & -0.2 & -0.1 & +0.1 & +0.2 & -0.2 & -0.1 & +0.1 & +0.2 \\
\hline \multirow{4}{*}{$\mu$-like } & $\bar{\delta}=0^{\circ}$ & 41.5 & 6.7 & 20.6 & 64.1 & 10.3 & 2.9 & 4.1 & 12.9 \\
\hline & $\bar{\delta}=90^{\circ}$ & 44.2 & 8.4 & 18.8 & 62.3 & 9.9 & 2.7 & 3.8 & 12.0 \\
\hline & $\bar{\delta}=180^{\circ}$ & 49.3 & 11.5 & 16.6 & 59.9 & 9.2 & 2.4 & 4.2 & 11.8 \\
\hline & $\bar{\delta}=270^{\circ}$ & 46.2 & 9.0 & 17.9 & 60.7 & 9.5 & 2.5 & 4.1 & 12.4 \\
\hline \multirow{4}{*}{$\mu+\mathrm{e}$-like } & $\bar{\delta}=0^{\circ}$ & 84.8 & 15.1 & 29.3 & 163 & 32.5 & 8.0 & 12.0 & 46.0 \\
\hline & $\bar{\delta}=90^{\circ}$ & 90.1 & 16.1 & 27.2 & 155 & 32.4 & 7.5 & 11.5 & 44.0 \\
\hline & $\bar{\delta}=180^{\circ}$ & 108 & 28.1 & 24.8 & 146 & 31.6 & 7.0 & 12.4 & 44.4 \\
\hline & $\bar{\delta}=270^{\circ}$ & 97.0 & 22.1 & 28.3 & 153 & 31.5 & 9.4 & 12.9 & 46.2 \\
\hline
\end{tabular}

Table 4. The dependence of the octant sensitivity on the input values $\bar{x}_{\mathrm{a}}$ and $\bar{\delta}$ after 1-year running of PINGU. The cases of both muon- and electron-like events for both $\mathrm{NH}$ and IH have been considered with event cuts $E_{\nu}>4 \mathrm{GeV}$ and $\cos \theta_{\mathrm{z}}<-0.4$.

We can see that the octant sensitivity for $\bar{x}_{\mathrm{a}}= \pm 0.1$ is much smaller than the one for $\bar{x}_{\mathrm{a}}= \pm 0.2$, by a factor around 4 , as expected. With smaller distance between the two mirrors, the difference due to the linear term is much smaller. And we can estimate the significance to scale roughly as $\Delta \chi_{\text {octant }}^{2} \propto x_{\mathrm{a}}^{2}$, according to (4.1), as verified by the results in table 4 .

Between the two mirrors, the octant sensitivity is always larger for positive $x_{\mathrm{a}}$ due to the same sign between $N_{\mu}^{(1)}$ and $N_{\mu}^{(5)}$. This makes the effective linear term coefficient in (4.7) larger with positive $\bar{x}_{\mathrm{a}}$, hence enhances the octant sensitivity. This trend is further enhanced by including the electron-like events which has only a negative linear term coefficient $N_{\mathrm{e}}^{(1)}$ but no quadratic term. With positive $\bar{x}_{\mathrm{a}}$, the event rates become smaller, explaining the further enhancement.

The dependence on the neutrino mass hierarchy is much easier to be understood. For NH, the octant sensitivity is much larger than that for IH, because the linear term coefficients $N_{\mu}^{(1)}$ and $N_{\mathrm{e}}^{(1)}$ have much larger magnitude for $\mathrm{NH}$.

There is small dependence on the CP phase. It comes from the cross term $x_{\mathrm{a}} \cos \delta^{\prime}$ whose coefficient $N_{\mu}^{(4)}$ is mainly positive for $\mathrm{NH}$ and negative for $\mathrm{IH}$ in the considered energy range $E_{\nu}>6 \mathrm{GeV}$, especially around $\cos \theta_{\mathrm{z}} \approx-0.6$, as shown in figure 7 (a). The effective linear term in (4.7) becomes,

$$
\left(N_{\mu}^{(1)}+\cos \bar{\delta}^{\prime} N_{\mu}^{(4)}+2 \bar{x}_{\mathrm{a}} N_{\mu}^{(5)}\right) \delta x_{\mathrm{a}}+\cdots .
$$

With nonzero $\bar{x}_{\mathrm{a}}$, especially when $\bar{x}_{\mathrm{a}} \approx \pm 0.2$, the quadratic term coefficient $N_{\mu}^{(5)}$ dominates. Cancellation between $\cos \bar{\delta}^{\prime} N_{\mu}^{(4)}$ and $2 \bar{x}_{\mathrm{a}} N_{\mu}^{(5)}$ happens if they have opposite signs, leading to smaller octant sensitivity as shown in table 4 . For $\mathrm{NH}$, the octant sensitivity is larger for $\cos \bar{\delta} \lesssim 0$ when $\bar{x}_{\mathrm{a}}$ is negative and $\cos \bar{\delta} \gtrsim 0$ when $\bar{x}_{\mathrm{a}}$ is positive. It is the opposite for IH due to the fact that $N_{\mu}^{(4)}$ receives an extra sign when the neutrino mass hierarchy switches. This trend remains for $\mathrm{NH}$ when the electron-like events are also included in the analysis. Note that the cross term does not have sizable effect on the uncertainty of measuring the atmospheric mixing angle but manifests itself in the octant sensitivity. 


\begin{tabular}{|c|l|ccc|ccc|}
\hline \multicolumn{2}{|c|}{} & \multicolumn{3}{|c|}{$\mathrm{NH}$} & \multicolumn{3}{c|}{$\mathrm{IH}$} \\
\hline \multirow{3}{*}{$E_{\nu}>6 \mathrm{GeV}$} & $\bar{x}_{\mathrm{a}}=-0.2$ & $\bar{x}_{\mathrm{a}}=0.0$ & $\bar{x}_{\mathrm{a}}=+0.2$ & $\bar{x}_{\mathrm{a}}=-0.2$ & $\bar{x}_{\mathrm{a}}=0.0$ & $\bar{x}_{\mathrm{a}}=+0.2$ \\
\hline & $\bar{\delta}=0^{\circ}$ & $23^{\circ}$ & $23^{\circ}$ & $22^{\circ}$ & $49^{\circ}$ & $48^{\circ}$ & $48^{\circ}$ \\
& $\bar{\delta}=90^{\circ}$ & $20^{\circ}$ & $20^{\circ}$ & $18^{\circ}$ & $41^{\circ}$ & $41^{\circ}$ & $38^{\circ}$ \\
& $\bar{\delta}=180^{\circ}$ & $23^{\circ}$ & $23^{\circ}$ & $21^{\circ}$ & $49^{\circ}$ & $48^{\circ}$ & $48^{\circ}$ \\
& $\bar{\delta}=270^{\circ}$ & $20^{\circ}$ & $20^{\circ}$ & $18^{\circ}$ & $41^{\circ}$ & $41^{\circ}$ & $38^{\circ}$ \\
\hline \multirow{3}{*}{$E_{\nu}>4 \mathrm{GeV}$} & $\bar{\delta}=0^{\circ}$ & $15^{\circ}$ & $14^{\circ}$ & $14^{\circ}$ & $32^{\circ}$ & $31^{\circ}$ & $32^{\circ}$ \\
& $\bar{\delta}=90^{\circ}$ & $13^{\circ}$ & $12^{\circ}$ & $11^{\circ}$ & $29^{\circ}$ & $29^{\circ}$ & $28^{\circ}$ \\
& $\bar{\delta}=180^{\circ}$ & $15^{\circ}$ & $14^{\circ}$ & $14^{\circ}$ & $32^{\circ}$ & $31^{\circ}$ & $32^{\circ}$ \\
& $\bar{\delta}=270^{\circ}$ & $13^{\circ}$ & $12^{\circ}$ & $11^{\circ}$ & $29^{\circ}$ & $29^{\circ}$ & $28^{\circ}$ \\
\hline
\end{tabular}

Table 5. Dependence of the uncertainty of measuring the CP phase $\delta$ on the input values $\bar{x}_{\mathrm{a}}$ and $\bar{\delta}$, after 10-years running of PINGU, for the NH (left 3 columns) and for the IH (right 3 columns). Both muon- and electron-like events in the region of $\cos \theta_{\mathrm{z}}<-0.4$ are used for $E_{\nu}>6 \mathrm{GeV}$ (upper 4 rows) and for $E_{\nu}>4 \mathrm{GeV}$ (lower 4 rows).

\subsection{Uncertainty of the CP phase}

In this section, we will show the capability of PINGU in measuring the CP phase $\delta$ in terms of $\chi_{\min }^{2}(\delta)$. The dependence of $\chi_{\min }^{2}(\delta)$ on $\delta$ comes from fixing $\delta$ and fitting the other five parameters to find the minimum. The uncertainty $\Delta(\delta)$ are then obtained according to the condition $\chi_{\min }^{2}(\delta)<1$ for each case of the input values, $\bar{x}_{\mathrm{a}}=0, \pm 0.2$, and $\bar{\delta}=0, \pm \pi / 2, \pi$, as well as both NH and IH. Since the $\delta$-dependent terms have tiny coefficients, $N_{\alpha}^{(2)}$ and $N_{\alpha}^{(3)}$, as shown in figure 5 and figure 6 , it is very challenging to determine the CP phase and can only be possible with a much longer time. We check the results after 10-years running of PINGU with both muon- and electron-like events.

As explained in section 2 and section 3 , the event rates depend on the CP phase $\delta$ only through the terms proportional to $\cos \delta$ and $\sin \delta$, since the coefficient $N_{\mu}^{(6)}$ of $\cos ^{2} \delta$ in the expansion (3.2) is negligibly small. Therefore, the $\delta$-dependence of $\chi_{\min }^{2}(\delta)$ can be approximated as a quadratic function of $\cos \delta$ and $\sin \delta$,

$$
\begin{aligned}
& \chi_{\min }^{2}(\delta)=\left(\begin{array}{ll}
\cos \delta-\cos \bar{\delta} & \sin \delta-\sin \bar{\delta}
\end{array}\right) V^{-1}\left(\begin{array}{c}
\cos \delta-\cos \bar{\delta} \\
\sin \delta-\sin \bar{\delta}
\end{array}\right)+\mathcal{O}\left((\delta-\bar{\delta})^{3}\right) \\
& =(-\sin \bar{\delta} \cos \bar{\delta}) V^{-1}\left(\begin{array}{c}
-\sin \bar{\delta} \\
\cos \bar{\delta}
\end{array}\right)(\delta-\bar{\delta})^{2}+\mathcal{O}\left((\delta-\bar{\delta})^{3}\right) \\
& =\left[\frac{\delta-\bar{\delta}}{\Delta(\delta)}\right]^{2}+\mathcal{O}\left((\delta-\bar{\delta})^{3}\right),
\end{aligned}
$$

where the covariance matrix $V$ is a $2 \times 2$ real symmetric matrix, and $\Delta(\delta)$ is the uncertainty on the $\mathrm{CP}$ phase. For various inputs, the results from exact $\chi^{2}$ minimization have been shown in table 5 .

Since the coefficients $N_{\alpha}^{(2)}$ and $N_{\alpha}^{(3)}$ are much smaller for IH, as shown in figure 5 and figure 6 , the resultant $\Delta(\delta)$ in table 5 is expected to be larger. Besides, there is very slight dependence on the input values of the atmospheric mixing angle and the $\mathrm{CP}$ phase, in contrast to the case at accelerator based neutrino experiments [122-124]. Since 
the $\delta$-dependence of the event rates occurs only through terms of $\cos \delta$ and $\sin \delta$, the $\chi_{\min }^{2}(\delta)$ function is a periodic function of $\delta$. It vanishes at $\delta=\bar{\delta}$ and has only one period of oscillation in the range of $[0,2 \pi]$ with peak around $|\delta-\bar{\delta}| \approx \pi$. With energy cut $E_{\nu}>6 \mathrm{GeV}$, the maximum value of $\chi_{\min }^{2}(\delta)$ is around $25 \sim 40$ for $\mathrm{NH}$, and just $6 \sim 8$ for $\mathrm{IH}$, depending on the input values of $\bar{x}_{\mathrm{a}}$ and $\bar{\delta}$. The value increases to around $12 \sim 16$ for IH if we include events down to $E_{\nu}=4 \mathrm{GeV}$. The function $\chi_{\min }^{2}(\delta)$ can be approximated by a quadratic function (4.10) of $\delta-\bar{\delta}$ up to more than $3 \sigma$ for all the results quoted in table 5 with the only exception of $E_{\nu}>6 \mathrm{GeV}$ for IH where it holds up only to around $2.5 \sigma$.

There is some slight $\bar{x}_{\mathrm{a}}$-dependence of the uncertainty $\Delta(\delta)$, which can then be read off directly by expressing the covariance matrix in terms of the decomposition coefficients, $N_{\alpha}^{(2)}+\bar{x}_{\mathrm{a}} N_{\alpha}^{(4)}$ and $N_{\alpha}^{(3)}$, for an input value of $x_{\mathrm{a}}=\bar{x}_{\mathrm{a}}$ as follows,

$$
\begin{aligned}
V^{-1} & =V_{\mu}^{-1}+V_{\mathrm{e}}^{-1}, \\
V_{\alpha}^{-1} & =\int \frac{d E_{\nu} d \cos \theta_{\mathrm{z}}}{N_{\alpha}^{(0)}+N_{\alpha}^{(1)} \bar{x}_{\mathrm{a}}+N_{\alpha}^{(5)} \bar{x}_{\mathrm{a}}^{2}}\left(1-\bar{x}_{\mathrm{a}}^{2}\right)\left(\begin{array}{cc}
{\left[N_{\alpha}^{(2)}+N_{\alpha}^{(4)} \bar{x}_{\mathrm{a}}\right]^{2}} & N_{\alpha}^{(3)}\left[N_{\alpha}^{(2)}+N_{\alpha}^{(4)} \bar{x}_{\mathrm{a}}\right] \\
N_{\alpha}^{(3)}\left[N_{\alpha}^{(2)}+N_{\alpha}^{(4)} \bar{x}_{\mathrm{a}}\right] & {\left[N_{\alpha}^{(3)}\right]^{2}}
\end{array}\right),
\end{aligned}
$$

where we neglect the small $\delta$-dependent terms in the denominator of (4.11b). Since $N_{\mu}^{(2)}$ and $N_{\mu}^{(4)}$ share the same sign in the considered energy range of $E_{\nu}>6 \mathrm{GeV}$, especially around $\cos \theta_{\mathrm{z}} \approx-0.8 \sim-0.6$, as shown in figure 5 , figure 6 , and figure 7 , the combination $N_{\mu}^{(2)}+$ $N_{\mu}^{(4)} \bar{x}_{\mathrm{a}}$ has larger magnitude when $\bar{x}_{\mathrm{a}}$ is positive. This explains the reduced uncertainty at $\bar{x}_{\mathrm{a}}=+0.2$. Note that there is an overall factor $1-\bar{x}_{\mathrm{a}}^{2}$ due to the modulation $\cos \delta^{\prime}=$ $\sqrt{1-x_{\mathrm{a}}^{2}} \cos \delta$ and $\sin \delta^{\prime}=\sqrt{1-x_{\mathrm{a}}^{2}} \sin \delta$. It can increase the CP phase uncertainty $\Delta(\delta)$ if the atmospheric mixing angle is not maximal, namely $1-x_{\mathrm{a}}^{2}<1$.

The $\bar{\delta}$ dependence of $\Delta(\delta)$ can be expressed as,

$$
\Delta(\delta)=\left[\left(V^{-1}\right)_{11} \sin ^{2} \bar{\delta}-2\left(V^{-1}\right)_{12} \sin \bar{\delta} \cos \bar{\delta}+\left(V^{-1}\right)_{22} \cos ^{2} \bar{\delta}\right]^{-1 / 2} .
$$

If the elements $\left(V^{-1}\right)_{11},\left(V^{-1}\right)_{12}$, and $\left(V^{-1}\right)_{22}$ have same size, the uncertainty on the $\mathrm{CP}$ phase, $\Delta(\delta)$, becomes $\bar{\delta}$-independent. Otherwise, $\Delta(\delta)$ would receive some variation. We can treat $\cos \delta$ and $\sin \delta$ as independent functions with nominal uncertainties $\Delta(\cos \delta)=\sqrt{V_{11}}, \Delta(\sin \delta)=\sqrt{V_{22}}$, and $V_{12}=\sqrt{V_{11}} \sqrt{V_{22}} \rho$. The above approximation for the covariance matrices gives, for $\bar{x}_{\mathrm{a}}=0$,

$$
\begin{array}{llll}
\Delta(\cos \delta)=0.65, & \Delta(\sin \delta)=0.97, & \rho=-0.29 & \text { for NH }(\mu \text {-like }), \\
\Delta(\cos \delta)=0.38, & \Delta(\sin \delta)=0.40, & \rho=-0.21 & \text { for NH }(\text { e-like }), \\
\Delta(\cos \delta)=0.33, & \Delta(\sin \delta)=0.37, & \rho=-0.23 & \text { for NH }(\mu+\mathrm{e}-\text { like }),
\end{array}
$$

and,

$$
\begin{array}{llll}
\Delta(\cos \delta)=1.43, & \Delta(\sin \delta)=1.79, & \rho=-0.27 & \text { for IH }(\mu \text {-like }), \\
\Delta(\cos \delta)=0.78, & \Delta(\sin \delta)=0.87, & \rho=-0.23 & \text { for IH }(\text { e-like }), \\
\Delta(\cos \delta)=0.69, & \Delta(\sin \delta)=0.78, & \rho=-0.24 & \text { for IH }(\mu+\text { e-like }),
\end{array}
$$


for the integration region of $6 \mathrm{GeV}<E_{\nu}<20 \mathrm{GeV}$ and $\cos \theta_{\mathrm{z}}<-0.4$ with 10 years running of PINGU.

From the results of (4.13) and (4.14), we find that the uncertainties $\Delta(\sin \delta)$ and $\Delta(\cos \delta)$ are rather large when only the muon-like events of $E_{\nu}>6 \mathrm{GeV}$ are used in the analysis. This is especially the case of $\mathrm{IH}$, for which the uncertainties are larger than unity even after 10 -years of running. In other words, the uncertainty $\Delta(\delta)$ can be rather broad. Therefore, the electron-like events are essential to measure the CP phase.

Generally speaking, $\Delta(\cos \delta)$ is slightly smaller than $\Delta(\sin \delta)$ due to larger magnitude of $N_{\alpha}^{(2)}$, resulting in slightly smaller uncertainties $\Delta(\delta)$ at $\bar{\delta}= \pm 90^{\circ}$ than those at $\bar{\delta}=0^{\circ}, 180^{\circ}$. In addition, the correlation turns out to be small $|\rho| \sim 0.2$ and negative for all the cases. From the combined results in (4.13) and (4.14), we can estimate the smallest and the largest uncertainty of $\Delta(\delta)$ as a function of $\bar{\delta}$,

$$
\begin{array}{lll}
\Delta(\delta)_{\max }=23^{\circ} & @ \quad \bar{\delta}=32^{\circ}, 212^{\circ} & \text { for } \mathrm{NH}(\mu+\mathrm{e}-\text { like }), \\
\Delta(\delta)_{\min }=17^{\circ} & @ \quad \bar{\delta}=122^{\circ}, 302^{\circ} \text { for } \mathrm{NH}(\mu+\mathrm{e}-\text { like }),
\end{array}
$$

for $\mathrm{NH}$, and

$$
\begin{array}{lll}
\Delta(\delta)_{\max }=48^{\circ} \quad @ \quad & \bar{\delta}=32^{\circ}, 212^{\circ} & \text { for IH }(\mu+\mathrm{e}-\text { like }), \\
\Delta(\delta)_{\min }=36^{\circ} \quad @ \quad \bar{\delta}=122^{\circ}, 302^{\circ} & \text { for IH }(\mu+\mathrm{e}-\text { like }),
\end{array}
$$

for IH. All the results quoted in table 5 lie within the above range. The largest differences are found to be about a few degrees for the $\mathrm{IH}$ case with $E_{\nu}>6 \mathrm{GeV}$.

\section{Conclusions and outlook}

In this work, we develop a general decomposition formalism in the propagation basis, which is extremely useful for the phenomenological study of neutrino oscillation. It can analytically separate the contributions of the three unknown parameters, namely, the neutrino mass hierarchy, the atmospheric mixing angle, and the CP phase. In this way, the pattern behind $\chi^{2}$ minimization can be revealed clearly, especially for atmospheric neutrino which experiences very complicated Earth matter profile. Hence, it can serve as a complementary tool to the neutrino oscillogram. The latter is designed for the overall pattern, especially the resonance behaviors, in the atmospheric neutrino oscillations, while our decomposition method can unveil more hidden structures behind the oscillogram. In addition, the decomposition method can apply generally to any type of neutrino oscillation experiment.

To illustrate the powerfulness of this decomposition formalism, we study in detail the ability of PINGU in determining the neutrino mass hierarchy, the atmospheric angle $\theta_{\mathrm{a}}$ and its octant, as well as the $\mathrm{CP}$ phase $\delta$ by measuring the oscillation pattern of atmospheric neutrinos. Both muon- and electron-like events have been considered. Our results suggest that PINGU has the potential to determine the mass hierarchy and the octant of the atmospheric mixing angle $\theta_{\mathrm{a}}$ within one year of operation if the neutrino energy and the zenith angle can be measured accurately in the region $E_{\nu}=4 \sim 20 \mathrm{GeV}$ and $\cos \theta_{\mathrm{z}}<-0.4$. The uncertainty of measuring the value of $\theta_{\mathrm{a}}$ can be reduced by a factor of $5 \sim 30$, while 
the determination of the $\mathrm{CP}$ phase $\delta$ is significantly more challenging. The dependence on the input values of the neutrino mass hierarchy, the atmospheric mixing angle, and the CP phase can be understood in our decomposition formalism. Our findings merit a serious investigation of the physics potential of PINGU with realistic detector response which we expect to be underway within the IceCube/PINGU Collaboration.

\section{Acknowledgments}

We thank Naotoshi Okamura for stimulating discussions in the early stage of our investigation. SFG is grateful to Hong-Jian He for kind support and Center for High Energy Physics of Tsinghua University (TUHEP), where part of this work is done, for hospitality. JSPS has provided SFG a generous postdoc fellowship, which is deeply appreciated, to continue the study at KEK. This work is supported in part by Grant-in-Aid for Scientific research (No. 25400287) from JSPS. CR is grateful for support from the Center for Cosmology and AstroParticle Physics (CCAPP) at The Ohio State University, where part of this work was performed. This work is supported in part by the Basic Science Research Program through the National Research Foundation of Korea funded by the Ministry of Education, Science and Technology (NRF-2013R1A1A1007068).

\section{A Atmospheric neutrino oscillation}

In this appendix, we introduce the input and method that we used to evaluate the atmospheric neutrino oscillation in this study. They include the atmospheric neutrino fluxes, interaction cross sections, and an energy-dependent effective detector volume, the Earth matter density profile and the numerical procedure.

\section{A.1 Atmospheric neutrino flux, cross sections and effective volume}

The atmospheric neutrino flux depends on many factors. First, it varies with the geographic location, mainly due to the earth magnetic field at the source regions. In addition, it dependents on the neutrino momentum direction, the zenith and the azimuth angles. Seasonal effects can also modulate the neutrino flux. For our study, we use an annual and azimuth angle averaged neutrino flux computed for the South Pole [102]. The earth magnetic field effect, which introduces the largest modification on neutrino fluxes, depending on the position, are mostly relevant for neutrino energies below the one considered in this study; therefore our results can be easily transferred to detectors at other geographic locations. With these factors taken into consideration, the neutrino flux is a function of neutrino energy and the zenith angle.

The energy dependence of the atmospheric neutrino flux is shown in figure 8(a). It can be seen that $\nu_{\mu}$ and $\bar{\nu}_{\mu}$ fluxes dominate over the $\nu_{\mathrm{e}}$ and $\bar{\nu}_{\mathrm{e}}$ fluxes. They drop very quickly with increasing energy, decreasing by four orders of magnitude from $E_{\nu}=1 \mathrm{GeV}$ to $E_{\nu}=20 \mathrm{GeV}$. For both flavors, the antineutrino flux is slightly smaller than the neutrino flux due to an asymmetry between the $\pi^{+}$and $\pi^{-}$production spectra in cosmic ray airshowers [102]. 
(a) Flux $\phi\left[\left(\mathrm{m}^{2} \text { sec sr GeV }\right)^{-1}\right]$

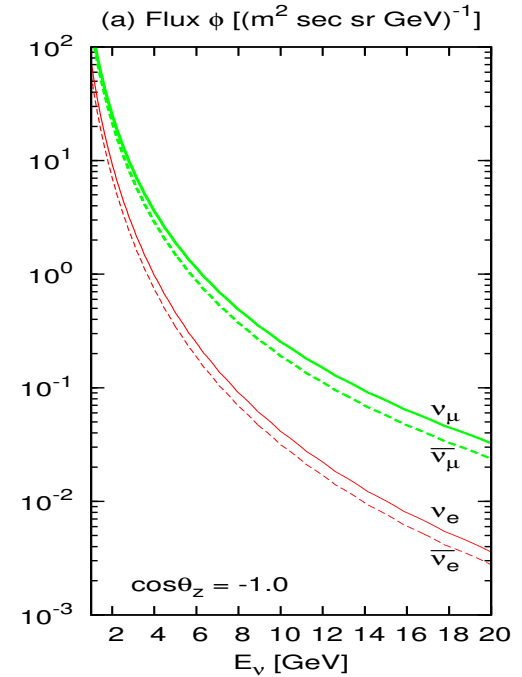

(b) $\phi \times \sigma\left[10^{-38}(\mathrm{sec} s r \mathrm{GeV})^{-1}\right]$

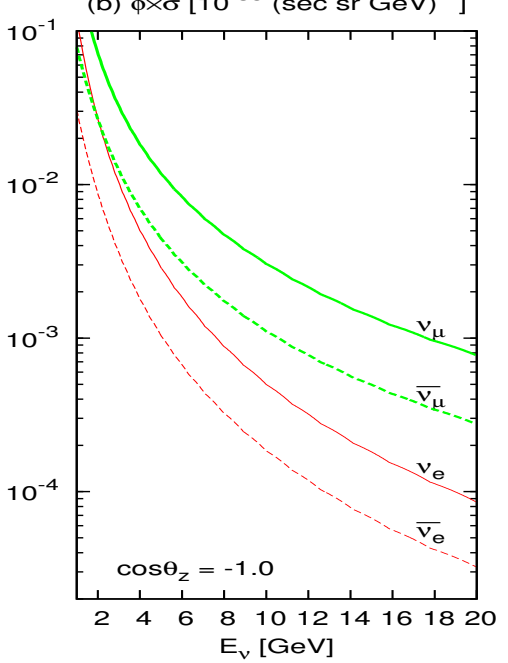

(c) $\phi \times \sigma \times \rho \times V_{\text {eff }}\left[(\text { year sr GeV) })^{-1}\right]$

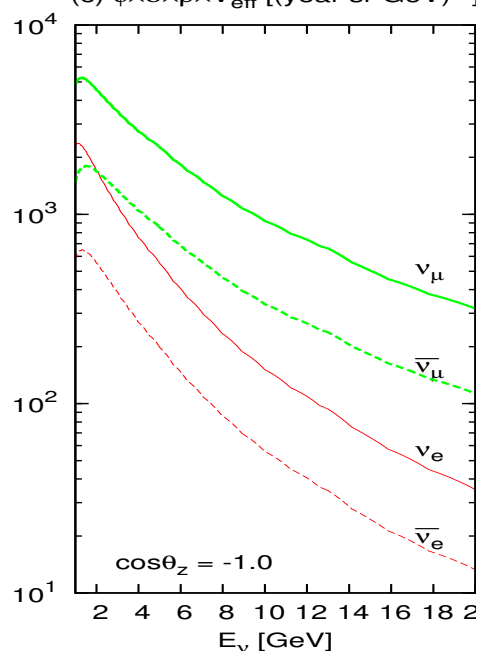

Figure 8. Energy dependence of (a) atmospheric neutrino fluxes $\phi$, (b) products of flux and cross section $\phi \times \sigma$, (c) products of flux, cross section and effective fiducial volume $\phi \times \sigma \times V_{\text {eff }}$, all at $\cos \theta_{\mathrm{z}}=-1.0$.

The atmospheric neutrino flux will be modulated by the neutrino interaction cross section with nuclei. In this study, we use the charged current (CC) cross sections generated by NEUGEN3 [104]. Since the cross sections increase linearly as function of neutrino energy $E_{\nu}$, higher energy event rates can be enhanced, by roughly an order of magnitude. In addition, the neutrino cross section is a factor of 2-3 larger than that of the antineutrino. The difference between the neutrino and antineutrino rates becomes even more significant when the atmospheric neutrino flux in figure $8(\mathrm{a})$ is multiplied with cross sections, as shown in figure $8(\mathrm{~b})$. This difference between the neutrino and antineutrino event rates is critical for the mass hierarchy determination with a detector incapable of telling leptons ( $\mu$ and $e$ ) from antileptons $(\bar{\mu}$ and $\bar{e})$, as will be made explicit in section A.4.

The PINGU effective fiducial volume used for our study assume a geometry of 20 additional strings within a radius of $75 \mathrm{~m}$ inside the DeepCore volume [105] with an interstring spacing of approximately $26 \mathrm{~m}$. It was required that at least 20 optical sensors would register a Cherenkov photon. At this level, events are assumed to be reconstructable. At the relevant energies in this study, the effective fiducial volume for electron and muon neutrinos is approximately equal [77]. Under this assumption, the fiducial volume is universal and will not affect the relative amount of electron and muon neutrinos/antineutrino event rates. The fiducial volume increases with energy, further increasing the event rates at higher energies. However, due to the much larger neutrino flux at lower energies, the highest event rates are expected to be detected at lower energies as can be seen in figure 8(c).

In figure 9, we compare the effective fiducial volume reported by the PINGU Collaboration [105], that we adopt in this study [see figure 8], and the one adopted in the pioneering work of Akhmedov et al. [66, 68]. In the left panel, the absolute values of both PINGU (solid curve) and the Akhmedov et al.'s paper (dashed curve) are shown while the ratio between the one used in Akhmedov et al.'s work and the PINGU curve is presented 

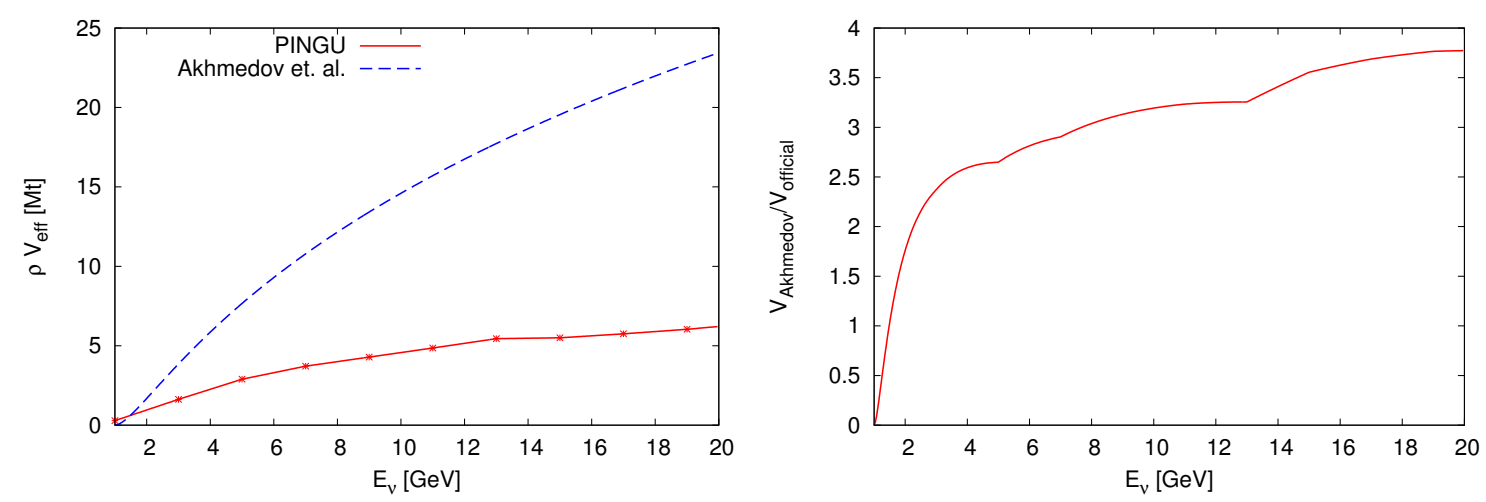

Figure 9. Comparison of the effective fiducial volume reported by the PINGU Collaboration [105], and the one adopted in the work of Akhmedov et al. [66, 68].

in the right panel. We can see that in most of the regions, the one implemented in the Akhmedov et al.'s paper is larger than the one from the PINGU Collaboration by a factor of around 3. It is only in the low energy end that this ratio decreases.

\section{A.2 Earth matter profile}

We use the preliminary Earth reference model (PREM) [98] to describe the Earth's matter density distribution. It represents the standard framework of interpreting the seismological data to determine the Earth matter density, assuming a spherically symmetric Earth without describing the chemical composition. The Earth radius varies from $6353 \mathrm{~km}$ to $6384 \mathrm{~km}$ due to rotational flattening, which distorts Earth's shape from that of an ideal sphere. The variation is relatively small $(0.5 \%)$ and hence it is a reasonable assumption to use a spherically symmetric Earth with radius $R=6371 \mathrm{~km}$. As the matter potential felt by neutrino depends on the electron density, we need to make assumptions about the chemical composition.

The matter potential felt by neutrino is proportional to the electron density,

$$
V(x)=\sqrt{2} G_{F} N_{\mathrm{e}}(x),
$$

while $V(x)$ receives an extra minus sign for antineutrino. The parameter $G_{F}$ is the Fermi constant and $N_{\mathrm{e}}(x)$ is the electron number density as a function of position, $x \equiv d / L\left(\theta_{\mathrm{z}}\right)$ with $d$ being the distance traversed by neutrino and $L\left(\theta_{z}\right)$ denoting the path length corresponding to the neutrino zenith angle $\theta_{\mathrm{z}}$. The electron number density $N_{\mathrm{e}}$ depends on the chemical composition of the Earth, which cannot be measured directly. It is approximated as a linear function of the matter density $\rho$ as $N_{\mathrm{e}}=Y \rho / m_{\mathrm{p}}$, where $m_{\mathrm{p}}$ is the nucleon mass. The coefficient $Y$ is the ratio between electron and nucleon number densities, given by $n_{\mathrm{p}} /\left(n_{\mathrm{p}}+n_{\mathrm{n}}\right)$ where $n_{\mathrm{p}}$ is the number density for proton and $n_{\mathrm{n}}$ the number density for neutron. Its value can vary significantly from light elements $Y \simeq 0.5$ to heavier elements, which increasingly have more neutrons per protons (for example $Y=0.466$ for $\mathrm{Fe}$ ). The core is expected to be dominated by iron, which takes an $85 \%$ share, rendering a smaller $Y_{\text {core }}=0.468$. For mantle, Oxygen $(Y=0.5)$, Magnesium $(Y=0.494)$, and 


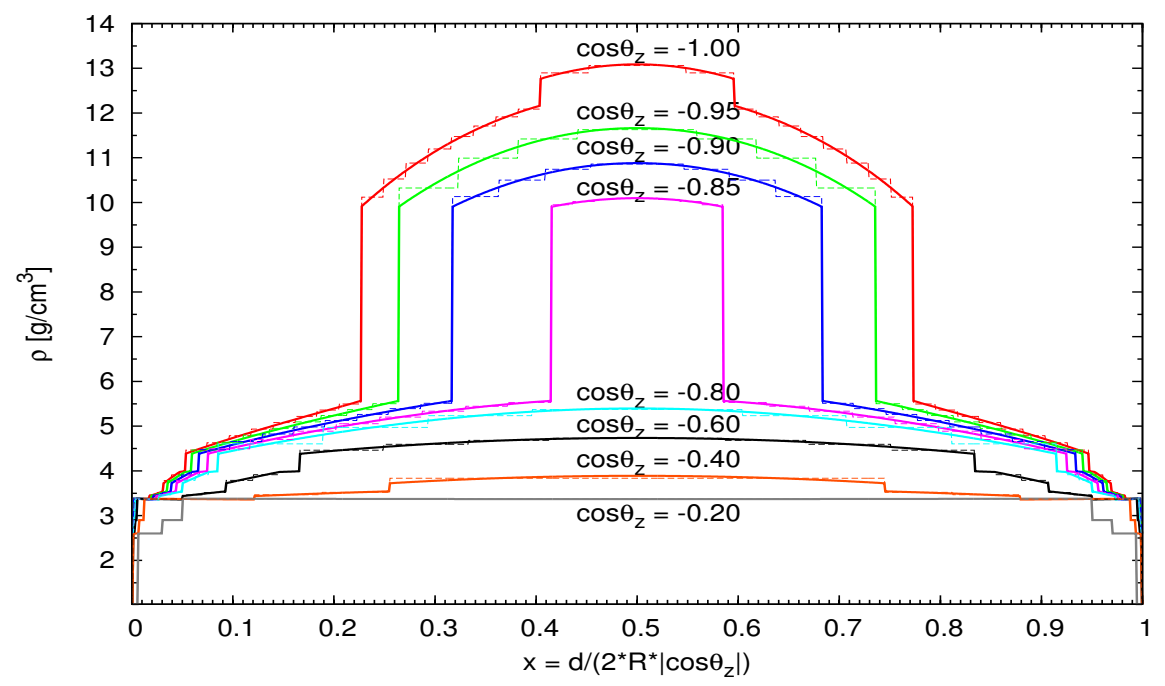

Figure 10. Realistic vs. sectioned PREM matter density profiles along paths with solid and dashed lines respectively. The Earth's radius is denoted as $R$ while $d$ is the path length of the neutrino traversing the Earth. For the sectioned profile, ten sublayers, from the inside to the outside, have been sliced into $n=2^{2,3,3,0,1,1,0,0,0,0}$ equal steps along the path. See appendix A.3 for details.

Silicon $(Y=0.498)$ take $44 \%, 23 \%$, and $21 \%$ of the total weight respectively, leading to a larger $Y_{\text {mantle }}=0.497$ [101]. The overall uncertainty in the averaged $Y_{\text {core }}$ and $Y_{\text {mantle }}$ is expected to be small, even if element abundances carry uncertainties of about $10 \%$ [101]. This is because the uncertainty in the averaged $Y$ 's is approximately a product of the $10 \%$ variation in the element abundances and the variation in $Y$ for individual element which also has around $10 \%$ variation. Hence, the estimated matter potential $V$ would receive an uncertainty around $1 \%$. In the current study, we omit this small uncertainty since it would not affect the potential of the atmospheric neutrino experiments at a qualitative level.

Figure 10 shows the matter density along different paths, denoted by the neutrino zenith angle $\theta_{\mathrm{z}}$. For $\cos \theta_{\mathrm{z}}=-1$, the path runs through the Earth center, corresponding to vertically up-going neutrino, with horizontal neutrinos denoted by $\cos \theta_{\mathrm{z}}=0$. The path length is related to the zenith angle as $L\left(\theta_{\mathrm{z}}\right)=2 R\left|\cos \theta_{\mathrm{z}}\right|$ and is symmetric with respect to the central point. For convenience, the horizontal axis in figure 10 is defined as $x \equiv d / L\left(\theta_{\mathrm{z}}\right)$ to make comparison between different paths. It should be noted that there is a big discontinuity at the boundary between the core and mantle. For $\cos \theta_{\mathrm{z}}=-1$, this boundary rests around $|x-0.5| \approx 0.27$ since the core's size $\left(R_{\text {core }}=3480 \mathrm{~km}\right)$ is almost half of the Earth's radius, $R=6371 \mathrm{~km}$. In addition, the core can be divided into the inner core and the outer one which are separated by a boundary at $r=1221.5 \mathrm{~km}$. The inner/outer core and mantle boundaries are very well known and have an uncertainty of less than $10 \mathrm{~km}[99,100]$. For the mantle, there are eight sublayers with boundaries at $r=(5701,5771,5971,6151,6346,6356,6368) \mathrm{km}$ respectively. Of these eight sublayers, the three outermost layers have constant matter density. Depending on the zenith angle $\theta_{z}$, neutrinos pass these various layers sequentially. 


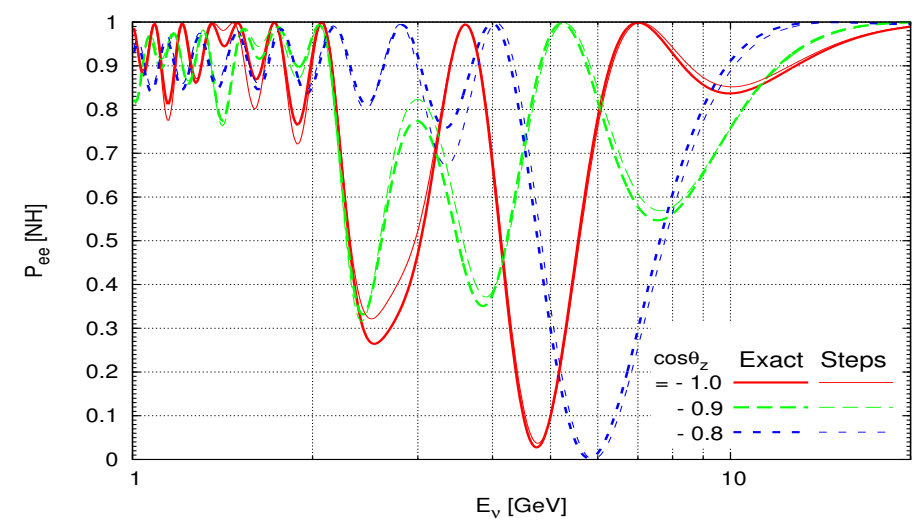

Figure 11. The oscillation probabilities $P_{\text {ee }}$ obtained with exact solution [thick lines] vs. averaged PREM steps for normal hierarchy. The results along three different paths $\cos \theta_{\mathrm{z}}=-1.0,-0.9,-0.8$ are shown as solid, dashed and dotted lines, respectively.

\section{A.3 Numerical method for oscillation amplitude matrix $S^{\prime}$ in the propagation basis}

Although the dependences of the oscillation probabilities on the atmospheric angle $\theta_{\mathrm{a}}$ and the CP phase $\delta$ can be expressed analytically, as elaborated in section 2 , the other oscillation parameters are still entangled with the matter effect inside the oscillation amplitudes $S_{i j}^{\prime}$ in the propagation basis, as explicitly shown in (2.8). Since the matter profile has a very complicated structure, depending on the neutrino zenith angle $\theta_{\mathrm{z}}$, it is necessary to find an accurate and efficient numerical method to evaluate $S_{\mathrm{ij}}^{\prime}$.

As an approximation, we first replace the PREM profile within each sublayer, as shown in figure 10, by a constant density averaged along the path. Within each constant potential, the oscillation amplitude matrix $A_{i}$ for three-neutrino oscillation can be evaluated exactly [125]. The full amplitude matrix is a sequential matrix product of these individual ones. In this way, we can keep the discontinuity between sublayers, especially the periodic mantle-core-mantle structure, which is important for parametric resonances. But the slowly varying behavior within each sublayer is averaged out. As an illustration, the resultant oscillation probabilities of the $\nu_{\mathrm{e}} \rightarrow \nu_{\mathrm{e}}$ channel for neutrino zenith angle $\cos \theta_{\mathrm{z}}=-1.0,-0.9,-0.8$, respectively, are shown in figure 11 with thin lines, in contrast to the exact solutions with thick lines. We can observe that this simple approximation shows very good agreements with the exact solution, especially that the peaks and troughs appear at almost exactly the same energies. In other words, the resonance features are maintained. But their amplitude can differ up to $10 \%$ which cannot be ignored. A more precise method is needed for a precision analysis such as $\chi^{2}$ minimization to obtain the physics potential of atmospheric neutrino oscillation experiments.

To account for the finer structure, we further divide each sublayer into several sections, within each the matter density is approximated by the averaged value along the path. Since the matter density has different slopes within different PREM layers, the number of sections is chosen accordingly as $n=2^{i}$ with $i=(2,3,3,0,1,1,0)$ for the density-varying 

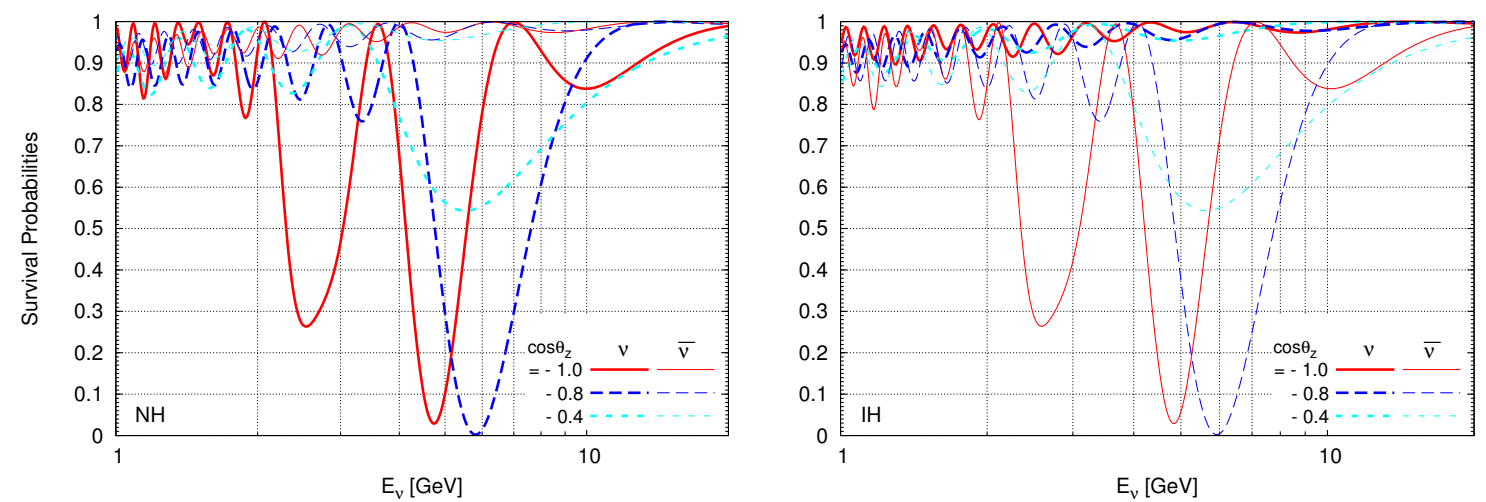

Figure 12. Neutrino oscillation probabilities $P_{\mathrm{ee}}$ (thick) and $P_{\bar{e} \bar{e}}$ (thin) vs. $E_{\nu}$ for NH (left) and $\mathrm{IH}$ (right), along the trajectories $\cos \theta_{\mathrm{z}}=-1,-0.8$ and -0.4 .

sublayers from the inner core to the outer crust. In this way, accuracy and efficiency can be balanced leading to an optimized program. In principle, with sublayers divided into more number of sections, the result would be closer to the exact solution. This is verified for linear potential by comparing with exact solution $[126,127]$ at various distances. For all the paths $\left(\cos \theta_{\mathrm{z}}\right)$ along the PREM matter distribution, we confirm that slicing the sublayers into finer sections gives no visible effects on the oscillation probabilities as we check by doubling the number of divisions with $n=2^{i+1}$. Hence, we call the solution with $n=(4,8,8,1,2,2,1)$ as the exact solution in this study. The difference in the probabilities is found to be in the order of $10^{-3}$, which can be safely ignored. The oscillation probabilities $P_{\text {ee }}$ of the exact solution are shown in figure 11 with thick lines.

\section{A.4 Normal hierarchy vs. inverted hierarchy}

The significant discontinuity in the matter density between the Earth's mantle and the core leads to an interesting pattern in the atmospheric neutrino oscillation probabilities. Along trajectories with $\cos \theta_{\mathrm{z}}<-0.84$, neutrinos experience a large jump in the potential causing parametric resonance [86-92] which is also known as oscillation length resonance [61-63, 9395]. With periodic matter density profile, the oscillation probability is largely enhanced. In addition, there is MSW resonance in the wide range of $\cos \theta_{\mathrm{z}}$ and $E_{\nu}[73-76]$ when neutrino crosses the mantle region. This makes the oscillation pattern of atmospheric neutrinos very sensitive to the neutrino mass hierarchy.

We briefly discuss the difference between $\mathrm{NH}$ and IH here. In figure 12, the electron neutrino (antineutrino) survival probabilities, $P_{\mathrm{ee}}\left(P_{\bar{e} \bar{e}}\right)$, along the paths with neutrino zenith angle $\cos \theta_{\mathrm{z}}=-1,-0.8$ and -0.4 are plotted for both $\mathrm{NH}$ in the left and IH in the right panel. For NH, strong oscillation patterns are found in the oscillation probability $P_{\mathrm{ee}}$ (thick curves) of neutrino, while the oscillation probability $P_{\bar{e} \bar{e}}$ (thin curves) of antineutrino has much smaller variation. This significant difference is because of the MSW resonance effect which can be demonstrated in the much simpler two-neutrino oscillation case. Under this simplified circumstance, the effective mixing angle can be analytically expressed as, $\sin 2 \widetilde{\theta}=\sin 2 \theta / \sqrt{\sin ^{2} 2 \theta+\left(\cos 2 \theta-2 E V / \delta m^{2}\right)^{2}}$, where $\theta$ and $\delta m^{2}$ are the true mixing 
angle and the true mass squared difference. The resonance happens at $\cos 2 \theta=2 E V / \delta m^{2}$, leading to a maximal effective mixing angle $\sin 2 \tilde{\theta}=1$. With a full treatment of threeneutrino oscillation, the probability resonates around $E=\cos 2 \theta_{\mathrm{ij}} \delta m_{\mathrm{ij}}^{2} / 2 V$, where $\theta_{\mathrm{ij}}$ and $\delta m_{\mathrm{ij}}^{2}$ are the relevant true mixing angle and the true mass squared difference. For the solar mass squared difference $\delta m_{12}^{2} \equiv m_{2}^{2}-m_{1}^{2}$, the resonance energy is around $100 \mathrm{MeV}$, with the typical matter potential in the mantle region, which is below the energy region considered in our studies. For the atmospheric mass squared difference $\delta m_{13}^{2} \equiv m_{3}^{2}-m_{1}^{2}$, instead, the resonance occurs around $4-6 \mathrm{GeV}$, which is within the accessible region of the investigated atmospheric neutrino oscillation in this study. It should be noted that the MSW resonance only occurs with $\delta m_{13}^{2}>0(\mathrm{NH})$ for neutrinos $(V>0)$, and with $\delta m_{13}^{2}<0$ (IH) for antineutrinos $(V<0)$, since $\cos 2 \theta_{13}=1-2 \sin ^{2} \theta_{13}>0$.

It is much simpler to determine the neutrino mass hierarchy if the detector is capable of distinguishing neutrinos from antineutrinos. With the MSW resonance around $2 \sim 7 \mathrm{GeV}$ observed in neutrinos rather than antineutrinos, then the mass hierarchy must be normal and vice versa. In other words, the existence or the absence of the MSW resonance can serve as a solid discriminator of the neutrino mass hierarchy. For a detector without the capability to distinguish neutrinos from antineutrinos, the MSW resonance could still be used to determine the mass hierarchy. This is made possible by the differences in the neutrino and antineutrino fluxes as well as their charged current cross sections discussed in section A.1. The hierarchy sensitivity obtained from the residual difference between NH and IH can still be sizable, if large enough event rates are collected by a huge underground detector such as PINGU.

Open Access. This article is distributed under the terms of the Creative Commons Attribution License (CC-BY 4.0), which permits any use, distribution and reproduction in any medium, provided the original author(s) and source are credited.

\section{References}

[1] T2K collaboration, K. Abe et al., Indication of electron neutrino appearance from an accelerator-produced off-axis muon neutrino beam, Phys. Rev. Lett. 107 (2011) 041801 [arXiv:1106.2822] [INSPIRE].

[2] MINOS collaboration, P. Adamson et al., Improved search for muon-neutrino to electron-neutrino oscillations in MINOS, Phys. Rev. Lett. 107 (2011) 181802 [arXiv:1108.0015] [INSPIRE].

[3] DOUBLE-CHOOZ collaboration, Y. Abe et al., Indication for the disappearance of reactor electron antineutrinos in the Double CHOOZ experiment, Phys. Rev. Lett. 108 (2012) 131801 [arXiv:1112.6353] [INSPIRE].

[4] DAYA BAY collaboration, F.P. An et al., Observation of electron-antineutrino disappearance at Daya Bay, Phys. Rev. Lett. 108 (2012) 171803 [arXiv:1203.1669] [InSPIRE].

[5] RENO collaboration, J.K. Ahn et al., Observation of reactor electron antineutrino disappearance in the RENO experiment, Phys. Rev. Lett. 108 (2012) 191802 [arXiv:1204.0626] [INSPIRE]. 
[6] DaYa Bay collaboration, F.P. An et al., Improved measurement of electron antineutrino disappearance at Daya Bay, Chin. Phys. C 37 (2013) 011001 [arXiv:1210.6327] [InSPIRE].

[7] H. Minakata, Phenomenology of future neutrino experiments with large $\theta_{13}$, Nucl. Phys. Proc. Suppl. 235-236 (2013) 173 [arXiv:1209.1690] [INSPIRE].

[8] S.T. Petcov and M. Piai, The LMA MSW solution of the solar neutrino problem, inverted neutrino mass hierarchy and reactor neutrino experiments, Phys. Lett. B 533 (2002) 94 [hep-ph/0112074] [INSPIRE].

[9] S. Choubey, S.T. Petcov and M. Piai, Precision neutrino oscillation physics with an intermediate baseline reactor neutrino experiment, Phys. Rev. D 68 (2003) 113006 [hep-ph/0306017] [INSPIRE].

[10] J. Learned, S.T. Dye, S. Pakvasa and R.C. Svoboda, Determination of neutrino mass hierarchy and $\theta_{13}$ with a remote detector of reactor antineutrinos, Phys. Rev. D 78 (2008) 071302 [hep-ex/0612022] [INSPIRE].

[11] L. Zhan, Y. Wang, J. Cao and L. Wen, Determination of the neutrino mass hierarchy at an intermediate baseline, Phys. Rev. D 78 (2008) 111103 [arXiv:0807.3203] [INSPIRE].

[12] M. Batygov et al., Prospects of neutrino oscillation measurements in the detection of reactor antineutrinos with a medium-baseline experiment, arXiv:0810.2580 [INSPIRE].

[13] L. Zhan, Y. Wang, J. Cao and L. Wen, Experimental requirements to determine the neutrino mass hierarchy using reactor neutrinos, Phys. Rev. D 79 (2009) 073007 [arXiv:0901.2976] [INSPIRE].

[14] P. Ghoshal and S.T. Petcov, Neutrino mass hierarchy determination using reactor antineutrinos, JHEP 03 (2011) 058 [arXiv:1011.1646] [INSPIRE].

[15] E. Ciuffoli, J. Evslin and X. Zhang, The neutrino mass hierarchy at reactor experiments now that $\theta_{13}$ is large, JHEP 03 (2013) 016 [arXiv:1208.1991] [INSPIRE].

[16] E. Ciuffoli, J. Evslin and X. Zhang, Mass hierarchy determination using neutrinos from multiple reactors, JHEP 12 (2012) 004 [arXiv:1209.2227] [INSPIRE].

[17] X. Qian et al., Mass hierarchy resolution in reactor anti-neutrino experiments: parameter degeneracies and detector energy response, Phys. Rev. D 87 (2013) 033005 [arXiv:1208.1551] [INSPIRE].

[18] P. Ghoshal and S.T. Petcov, Addendum: Neutrino mass hierarchy determination using reactor antineutrinos, JHEP 09 (2012) 115 [arXiv:1208.6473] [INSPIRE].

[19] X. Qian et al., Statistical evaluation of experimental determinations of neutrino mass hierarchy, Phys. Rev. D 86 (2012) 113011 [arXiv:1210.3651] [INSPIRE].

[20] Y.-F. Li, J. Cao, Y. Wang and L. Zhan, Unambiguous determination of the neutrino mass hierarchy using reactor neutrinos, Phys. Rev. D 88 (2013) 013008 [arXiv:1303.6733] [INSPIRE].

[21] S.-F. Ge, K. Hagiwara, N. Okamura and Y. Takaesu, Determination of mass hierarchy with medium baseline reactor neutrino experiments, JHEP 05 (2013) 131 [arXiv:1210.8141] [INSPIRE].

[22] H. Minakata and H. Nunokawa, Exploring neutrino mixing with low-energy superbeams, JHEP 10 (2001) 001 [hep-ph/0108085] [INSPIRE].

[23] V. Barger, D. Marfatia and K. Whisnant, Breaking eight fold degeneracies in neutrino CP-violation, mixing and mass hierarchy, Phys. Rev. D 65 (2002) 073023 [hep-ph/0112119] [INSPIRE]. 
[24] P. Huber, M. Lindner and W. Winter, Superbeams versus neutrino factories, Nucl. Phys. B 645 (2002) 3 [hep-ph/0204352] [INSPIRE].

[25] H. Minakata, H. Nunokawa and S.J. Parke, The complementarity of eastern and western hemisphere long baseline neutrino oscillation experiments, Phys. Rev. D 68 (2003) 013010 [hep-ph/0301210] [INSPIRE].

[26] VLBL Study Group H2B-1 collaboration, H.-s. Chen et al., Prospect of a very long baseline neutrino oscillation experiment: HIPA to Beijing, hep-ph/0104266 [INSPIRE].

[27] VLBL Study Group H2B-4 collaboration, Y.F. Wang, K. Whisnant, Z.-h. Xiong, J.M. Yang and B.-L. Young, Probing neutrino oscillations jointly in long and very long baseline experiments, Phys. Rev. D 65 (2002) 073021 [hep-ph/0111317] [INSPIRE].

[28] M. Aoki et al., Prospects of very long baseline neutrino oscillation experiments with the KEK/JAERI high intensity proton accelerator, Phys. Rev. D 67 (2003) 093004 [hep-ph/0112338] [INSPIRE].

[29] M. Ishitsuka, T. Kajita, H. Minakata and H. Nunokawa, Resolving neutrino mass hierarchy and $C P$ degeneracy by two identical detectors with different baselines, Phys. Rev. D 72 (2005) 033003 [hep-ph/0504026] [INSPIRE].

[30] K. Hagiwara, N. Okamura and K.-i. Senda, Solving the neutrino parameter degeneracy by measuring the T2K off-axis beam in Korea, Phys. Lett. B 637 (2006) 266 [Erratum ibid. B 641 (2006) 491] [hep-ph/0504061] [INSPIRE].

[31] K. Hagiwara, N. Okamura and K.-i. Senda, Physics potential of T2KK: an extension of the T2K neutrino oscillation experiment with a far detector in Korea, Phys. Rev. D 76 (2007) 093002 [hep-ph/0607255] [INSPIRE].

[32] T. Kajita, H. Minakata, S. Nakayama and H. Nunokawa, Resolving eight-fold neutrino parameter degeneracy by two identical detectors with different baselines, Phys. Rev. D 75 (2007) 013006 [hep-ph/0609286] [INSPIRE].

[33] K. Hagiwara and N. Okamura, Solving the degeneracy of the lepton-flavor mixing angle $\theta_{\mathrm{ATM}}$ by the T2KK two detector neutrino oscillation experiment, JHEP 01 (2008) 022 [hep-ph/0611058] [INSPIRE].

[34] K. Hagiwara and N. Okamura, Re-evaluation of the T2KK physics potential with simulations including backgrounds, JHEP 07 (2009) 031 [arXiv: 0901.1517] [INSPIRE].

[35] S. Prakash, S.K. Raut and S.U. Sankar, Getting the best out of T2K and NOvA, Phys. Rev. D 86 (2012) 033012 [arXiv:1201.6485] [INSPIRE].

[36] K. Hagiwara, T. Kiwanami, N. Okamura and K.-i. Senda, Physics potential of neutrino oscillation experiment with a far detector in Oki Island along the T2K baseline, JHEP 06 (2013) 036 [arXiv:1209.2763] [INSPIRE].

[37] S. Dusini et al., CP violation and mass hierarchy at medium baselines in the large $\theta_{13}$ era, Eur. Phys. J. C 73 (2013) 2392 [arXiv:1209.5010] [INSPIRE].

[38] K. Abe et al., Letter of intent: the Hyper-Kamiokande experiment - detector design and physics potential, arXiv:1109.3262 [INSPIRE].

[39] Hyper-Kamiokande Working Group collaboration, E. Kearns et al., Hyper-Kamiokande physics opportunities, arXiv:1309.0184 [INSPIRE].

[40] A. Rubbia, Experiments for CP-violation: a giant liquid argon scintillation, Cerenkov and charge imaging experiment?, hep-ph/0402110 [INSPIRE]. 
[41] A. Ereditato and A. Rubbia, Conceptual design of a scalable multi-kton superconducting magnetized liquid argon TPC, Nucl. Phys. Proc. Suppl. 155 (2006) 233 [hep-ph/0510131] [INSPIRE].

[42] G. Battistoni, A. Ferrari, C. Rubbia, P.R. Sala and F. Vissani, Atmospheric neutrinos in a large liquid argon detector, hep-ph/0604182 [INSPIRE].

[43] A. Bueno et al., Nucleon decay searches with large liquid argon TPC detectors at shallow depths: atmospheric neutrinos and cosmogenic backgrounds, JHEP 04 (2007) 041 [hep-ph/0701101] [INSPIRE].

[44] M. Blennow and T. Schwetz, Identifying the neutrino mass ordering with INO and NOvA, JHEP 08 (2012) 058 [Erratum ibid. 11 (2012) 098] [arXiv:1203. 3388] [INSPIRE].

[45] A. Ghosh, T. Thakore and S. Choubey, Determining the neutrino mass hierarchy with INO, T2K, NOvA and reactor experiments, JHEP 04 (2013) 009 [arXiv:1212.1305] [INSPIRE].

[46] T. Thakore, A. Ghosh, S. Choubey and A. Dighe, The reach of INO for atmospheric neutrino oscillation parameters, JHEP 05 (2013) 058 [arXiv:1303.2534] [INSPIRE].

[47] O. Mena, I. Mocioiu and S. Razzaque, Neutrino mass hierarchy extraction using atmospheric neutrinos in ice, Phys. Rev. D 78 (2008) 093003 [arXiv:0803.3044] [INSPIRE].

[48] A. Samanta, Exceptional sensitivities of neutrino mixing parameters with atmospheric neutrinos, Phys. Rev. D 80 (2009) 113003 [arXiv:0812.4639] [INSPIRE].

[49] A. Samanta, Discrimination of mass hierarchy with atmospheric neutrinos at a magnetized muon detector, Phys. Rev. D 81 (2010) 037302 [arXiv:0907.3540] [INSPIRE].

[50] E. Fernandez-Martinez, G. Giordano, O. Mena and I. Mocioiu, Atmospheric neutrinos in ice and measurement of neutrino oscillation parameters, Phys. Rev. D 82 (2010) 093011 [arXiv: 1008.4783] [INSPIRE].

[51] V. Barger et al., Neutrino mass hierarchy and octant determination with atmospheric neutrinos, Phys. Rev. Lett. 109 (2012) 091801 [arXiv:1203.6012] [INSPIRE].

[52] IceCube collaboration, R. Abbasi et al., The design and performance of IceCube DeepCore, Astropart. Phys. 35 (2012) 615 [arXiv:1109.6096] [inSPIRE].

[53] ICECuBE collaboration, M.G. Aartsen et al., Measurement of atmospheric neutrino oscillations with IceCube, Phys. Rev. Lett. 111 (2013) 081801 [arXiv:1305.3909] [INSPIRE].

[54] ICECube collaboration, C.H. Ha, Detection of cascades induced by atmospheric neutrinos in the 79-string IceCube detector, arXiv:1209.0698 [INSPIRE].

[55] ICECuBE collaboration, M.G. Aartsen et al., Measurement of the atmospheric $\nu_{e}$ flux in IceCube, Phys. Rev. Lett. 110 (2013) 151105 [arXiv:1212.4760] [INSPIRE].

[56] IcECube collaboration, A. Achterberg et al., First year performance of the IceCube Neutrino Telescope, Astropart. Phys. 26 (2006) 155 [astro-ph/0604450] [INSPIRE].

[57] D.J. Koskinen, IceCube-DeepCore-PINGU: fundamental neutrino and dark matter physics at the South Pole, Mod. Phys. Lett. A 26 (2011) 2899 [INSPIRE].

[58] PINGU collaboration, D. Cowen, PINGU and the neutrino mass hierarchy. Fundamental particle physics in the ice with atmospheric neutrinos, talk given at the P5 Workshop on the Future of High Energy Physics, SLAC U.S.A., 2-4 Dec 2013, https://indico.bnl.gov/ getFile.py $/$ access?contribId $=6 \&$ sessionId $=1 \&$ resId $=0 \&$ materialId $=$ slides\&confId $=688$. 
[59] S.T. Petcov, New enhancement mechanism of the transitions in the Earth of the solar and atmospheric neutrinos crossing the Earth core, Nucl. Phys. Proc. Suppl. 77 (1999) 93 [hep-ph/9809587] [INSPIRE].

[60] S.T. Petcov, The oscillation length resonance in the transitions of solar and atmospheric neutrinos crossing the Earth core, hep-ph/9811205 [INSPIRE].

[61] M. Chizhov, M. Maris and S.T. Petcov, On the oscillation length resonance in the transitions of solar and atmospheric neutrinos crossing the Earth core, hep-ph/9810501 [INSPIRE].

[62] M.V. Chizhov and S.T. Petcov, New conditions for a total neutrino conversion in a medium, Phys. Rev. Lett. 83 (1999) 1096 [hep-ph/9903399] [INSPIRE].

[63] M.V. Chizhov and S.T. Petcov, Enhancing mechanisms of neutrino transitions in a medium of nonperiodic constant density layers and in the Earth, Phys. Rev. D 63 (2001) 073003 [hep-ph/9903424] [INSPIRE].

[64] E.K. Akhmedov, M. Maltoni and A.Y. Smirnov, 1-3 leptonic mixing and the neutrino oscillograms of the Earth, JHEP 05 (2007) 077 [hep-ph/0612285] [INSPIRE].

[65] E.K. Akhmedov, M. Maltoni and A.Y. Smirnov, Neutrino oscillograms of the Earth: effects of 1-2 mixing and CP-violation, JHEP 06 (2008) 072 [arXiv:0804.1466] [INSPIRE].

[66] E.K. Akhmedov, S. Razzaque and A.Y. Smirnov, Mass hierarchy, 2-3 mixing and CP-phase with huge atmospheric neutrino detectors, JHEP 02 (2013) 082 [Erratum ibid. 07 (2013) 026] [arXiv: 1205.7071] [INSPIRE].

[67] S.K. Agarwalla, T. Li, O. Mena and S. Palomares-Ruiz, Exploring the Earth matter effect with atmospheric neutrinos in ice, arXiv:1212.2238 [INSPIRE].

[68] M. Ribordy and A.Y. Smirnov, Improving the neutrino mass hierarchy identification with inelasticity measurement in PINGU and ORCA, Phys. Rev. D 87 (2013) 113007 [arXiv: 1303.0758] [INSPIRE].

[69] W. Winter, Neutrino mass hierarchy determination with IceCube-PINGU, Phys. Rev. D 88 (2013) 013013 [arXiv:1305.5539] [INSPIRE].

[70] M. Blennow and T. Schwetz, Determination of the neutrino mass ordering by combining PINGU and Daya Bay II, JHEP 09 (2013) 089 [arXiv:1306.3988] [INSPIRE].

[71] A. Blake, J. Chapman and M. Thomson, A Bayesian technique for improving the sensitivity of the atmospheric neutrino L/E analysis, Nucl. Instrum. Meth. A 707 (2013) 127 [arXiv: 1208.2899] [INSPIRE].

[72] D. Franco et al., Mass hierarchy discrimination with atmospheric neutrinos in large volume ice/water Cherenkov detectors, JHEP 04 (2013) 008 [arXiv:1301.4332] [INSPIRE].

[73] L. Wolfenstein, Neutrino oscillations in matter, Phys. Rev. D 17 (1978) 2369 [INSPIRE].

[74] L. Wolfenstein, Neutrino oscillations and stellar collapse, Phys. Rev. D 20 (1979) 2634 [INSPIRE].

[75] S.P. Mikheev and A.Y. Smirnov, Resonance amplification of oscillations in matter and spectroscopy of solar neutrinos, Sov. J. Nucl. Phys. 42 (1985) 913

[Yad. Fiz. 42 (1985) 1441] [INSPIRE].

[76] S.P. Mikheev and A.Y. Smirnov, Neutrino oscillations in a variable density medium and neutrino bursts due to the gravitational collapse of stars, Sov. Phys. JETP 64 (1986) 4 [Zh. Eksp. Teor. Fiz. 91 (1986) 7] [arXiv:0706.0454] [INSPIRE]. 
[77] J. Tang and W. Winter, Requirements for a new detector at the South Pole receiving an accelerator neutrino beam, JHEP 02 (2012) 028 [arXiv:1110.5908] [INSPIRE].

[78] IceCube collaboration, T. DeYoung, Particle physics in ice with IceCube DeepCore, Nucl. Instrum. Meth. A 692 (2012) 180 [arXiv:1112.1053] [InSPIRE].

[79] R. Allahverdi and K. Richardson, Distinguishing among dark matter annihilation channels with neutrino telescopes, Phys. Rev. D 85 (2012) 113012 [arXiv:1201.6603] [INSPIRE].

[80] J.M. Cornell and S. Profumo, Earthly probes of the smallest dark matter halos, JCAP 06 (2012) 011 [arXiv:1203.1100] [INSPIRE].

[81] J.L. Hewett et al., Fundamental physics at the intensity frontier, arXiv:1205.2671 [INSPIRE].

[82] A. Karle, Neutrino astronomy - a review of future experiments, Nucl. Phys. Proc. Suppl. 235-236 (2013) 364 [arXiv:1210.2058] [INSPIRE].

[83] C. Rott, Review of indirect WIMP search experiments, Nucl. Phys. Proc. Suppl. 235-236 (2013) 413 [arXiv:1210.4161] [INSPIRE].

[84] IceCube and PINGU collaborations, M.G. Aartsen et al., PINGU sensitivity to the neutrino mass hierarchy, arXiv:1306.5846 [INSPIRE].

[85] Km3Net collaboration, P. Coyle, ORCA: Oscillation Research with Cosmics in the Abyss, contribution to the European Strategy Preparatory Group Open Symposium, Krakow Poland, 10-12 Sep 2012.

[86] E.K. Akhmedov, Neutrino oscillations in inhomogeneous matter (in Russian), Sov. J. Nucl. Phys. 47 (1988) 301 [Yad. Fiz. 47 (1988) 475] [inSPIRE].

[87] P.I. Krastev and A.Y. Smirnov, Parametric effects in neutrino oscillations, Phys. Lett. B 226 (1989) 341 [INSPIRE].

[88] Q.Y. Liu and A.Y. Smirnov, Neutrino mass spectrum with $\nu_{\mu} \rightarrow \nu_{s}$ oscillations of atmospheric neutrinos, Nucl. Phys. B 524 (1998) 505 [hep-ph/9712493] [InSPIRE].

[89] Q.Y. Liu, S.P. Mikheyev and A.Y. Smirnov, Parametric resonance in oscillations of atmospheric neutrinos?, Phys. Lett. B 440 (1998) 319 [hep-ph/9803415] [INSPIRE].

[90] E.K. Akhmedov, Parametric resonance of neutrino oscillations and passage of solar and atmospheric neutrinos through the Earth, Nucl. Phys. B 538 (1999) 25 [hep-ph/9805272] [INSPIRE].

[91] E.K. Akhmedov, A. Dighe, P. Lipari and A.Y. Smirnov, Atmospheric neutrinos at Super-Kamiokande and parametric resonance in neutrino oscillations,

Nucl. Phys. B 542 (1999) 3 [hep-ph/9808270] [INSPIRE].

[92] E.K. Akhmedov, M. Maltoni and A.Y. Smirnov, Oscillations of high energy neutrinos in matter: precise formalism and parametric resonance, Phys. Rev. Lett. 95 (2005) 211801 [hep-ph/0506064] [INSPIRE].

[93] Q.Y. Liu, M. Maris and S.T. Petcov, Study of the day-night effect for the Super-Kamiokande detector. I. Time-averaged solar neutrino survival probability, Phys. Rev. D 56 (1997) 5991 [hep-ph/9702361] [INSPIRE].

[94] M. Maris and S.T. Petcov, Study of the day-night effect for the Super-Kamiokande detector. II. Electron spectrum deformations and day-night asymmetries, Phys. Rev. D 56 (1997) 7444 [hep-ph/9705392] [INSPIRE]. 
[95] S.T. Petcov, Diffractive-like (or parametric resonance-like?) enhancement of the Earth (day-night) effect for solar neutrinos crossing the Earth core, Phys. Lett. B 434 (1998) 321 [hep-ph/9805262] [INSPIRE].

[96] A. Chatterjee, P. Ghoshal, S. Goswami and S.K. Raut, Octant sensitivity for large $\theta_{13}$ in atmospheric and long baseline neutrino experiments, JHEP 06 (2013) 010 [arXiv:1302.1370] [INSPIRE].

[97] M. Ghosh, P. Ghoshal, S. Goswami and S.K. Raut, Can atmospheric neutrino experiments provide the first hint of leptonic CP violation?, Phys. Rev. D 89 (2014) 011301 [arXiv:1306.2500] [INSPIRE].

[98] A.M. Dziewonski and D.L. Anderson, Preliminary reference Earth model, Phys. Earth Planet. In. 25 (1981) 297 [InSPIRE].

[99] T.G. Masters and P.M. Shearer, Seismic models of the Earth, in Global Earth physics, T.J. Ahrens ed., American Geophysical Union, Washington U.S.A. (1995), doi: 10.1029/RF001p0088.

[100] W.F. McDonough, Compositional model for the Earth's core, in Treatise on geochemistry. Volume 2: The mantle and core, R.W. Carlson ed., Elsevier-Pergamon, Oxford U.K. (2003), pp. 547-568, doi: 10.1016/B0-08-043751-6/02015-6.

[101] W.F. McDonough and R. Arevalo Jr., Uncertainties in the composition of Earth, its core and silicate sphere, J. Phys. Conf. Ser. 136 (2008) 022006.

[102] M. Sajjad Athar, M. Honda, T. Kajita, K. Kasahara and S. Midorikawa, Atmospheric neutrino flux at INO, South Pole and Pyhäsalmi, Phys. Lett. B 718 (2013) 1375 [arXiv:1210.5154] [INSPIRE].

[103] Y.S. Jeong and M.H. Reno, Tau neutrino and antineutrino cross sections, Phys. Rev. D 82 (2010) 033010 [arXiv:1007.1966] [INSPIRE].

[104] H. Gallagher, The NEUGEN neutrino event generator, Nucl. Phys. Proc. Suppl. 112 (2002) 188 [inSPIRE].

[105] ICECuBE collaboration, A. Gross, Studies on the sensitivity to measure neutrino mass hierarchy with PINGU, talk given at the 33rd International Cosmic Ray Conference, Rio de Janeiro Brazil, 2-9 Jul 2013.

[106] E.K. Akhmedov, A. Dighe, P. Lipari and A.Y. Smirnov, Atmospheric neutrinos at Super-Kamiokande and parametric resonance in neutrino oscillations, Nucl. Phys. B 542 (1999) 3 [hep-ph/9808270] [INSPIRE].

[107] H. Yokomakura, K. Kimura and A. Takamura, Overall feature of CP dependence for neutrino oscillation probability in arbitrary matter profile, Phys. Lett. B 544 (2002) 286 [hep-ph/0207174] [INSPIRE].

[108] S.T. Petcov, An analytic description of three neutrino oscillations in matter with varying density, Phys. Lett. B 214 (1988) 259 [InSPIRE].

[109] Particle Data Group collaboration, J. Beringer et al., Review of particle physics, Phys. Rev. D 86 (2012) 010001 [INSPIRE].

[110] E.K. Akhmedov, P. Huber, M. Lindner and T. Ohlsson, T violation in neutrino oscillations in matter, Nucl. Phys. B 608 (2001) 394 [hep-ph/0105029] [InSPIRE].

[111] P.A.N. Machado, H. Minakata, H. Nunokawa and R. Zukanovich Funchal, Combining accelerator and reactor measurements of $\theta_{13}$ : the first result, JHEP 05 (2012) 023 [arXiv:1111.3330] [INSPIRE]. 
[112] MINOS collaboration, G. Barr, MINOS neutrino oscillation results, talk given at ICHEP2012 - 36th International Conference for High Energy Physics, Melbourne Australia, 4-11 Jul 2012.

[113] D.V. Forero, M. Tortola and J.W.F. Valle, Global status of neutrino oscillation parameters after Neutrino-2012, Phys. Rev. D 86 (2012) 073012 [arXiv: 1205.4018] [INSPIRE].

[114] G.L. Fogli et al., Global analysis of neutrino masses, mixings and phases: entering the era of leptonic CP-violation searches, Phys. Rev. D 86 (2012) 013012 [arXiv:1205.5254] [INSPIRE].

[115] M.C. Gonzalez-Garcia, M. Maltoni, J. Salvado and T. Schwetz, Global fit to three neutrino mixing: critical look at present precision, JHEP 12 (2012) 123 [arXiv:1209.3023] [INSPIRE].

[116] T. Schwetz, What is the probability that $\theta_{13}$ and CP-violation will be discovered in future neutrino oscillation experiments?, Phys. Lett. B 648 (2007) 54 [hep-ph/0612223] [INSPIRE].

[117] G. Cowan, K. Cranmer, E. Gross and O. Vitells, Asymptotic formulae for likelihood-based tests of new physics, Eur. Phys. J. C 71 (2011) 1554 [arXiv:1007.1727] [INSPIRE].

[118] X. Qian et al., Statistical evaluation of experimental determinations of neutrino mass hierarchy, Phys. Rev. D 86 (2012) 113011 [arXiv:1210.3651] [INSPIRE].

[119] S.-F. Ge, K. Hagiwara, N. Okamura and Y. Takaesu, Determination of mass hierarchy with medium baseline reactor neutrino experiments, JHEP 05 (2013) 131 [arXiv:1210.8141] [INSPIRE].

[120] E. Ciuffoli, J. Evslin and X. Zhang, Confidence in a neutrino mass hierarchy determination, JHEP 01 (2014) 095 [arXiv: 1305.5150] [INSPIRE].

[121] MINUIT, http://seal.web.cern.ch/seal/work-packages/mathlibs/minuit/.

[122] V. Barger, D. Marfatia and K. Whisnant, Breaking eight fold degeneracies in neutrino CP-violation, mixing and mass hierarchy, Phys. Rev. D 65 (2002) 073023 [hep-ph/0112119] [INSPIRE].

[123] O. Mena and S.J. Parke, Untangling CP-violation and the mass hierarchy in long baseline experiments, Phys. Rev. D 70 (2004) 093011 [hep-ph/0408070] [INSPIRE].

[124] H. Minakata and S. Uchinami, Parameter degeneracy in neutrino oscillation - solution network and structural overview, JHEP 04 (2010) 111 [arXiv:1001.4219] [INSPIRE].

[125] B. Dziewit, S. Zajac and M. Zralek, Majorana neutrino mass matrix with CP symmetry breaking, Acta Phys. Polon. B 42 (2011) 2509 [arXiv:1204.3665] [InSPIRE].

[126] W.C. Haxton, Analytic treatments of matter enhanced solar neutrino oscillations, Phys. Rev. D 35 (1987) 2352 [INSPIRE].

[127] S.T. Petcov, On the nonadiabatic neutrino oscillations in matter, Phys. Lett. B 191 (1987) 299 [INSPIRE]. 